\title{
On guaranteed parameter estimation of a multiparameter linear regression process ${ }^{1,2}$
}

\author{
Uwe Küchler \\ Institute of Mathematics \\ Humboldt University Berlin \\ Unter den Linden 6, D-10099 \\ Berlin, Germany
}

\author{
Vyacheslav A. Vasiliev \\ Department of Applied Mathematics \\ and Cybernetics \\ Tomsk State University \\ Lenina 36, 634050 Tomsk, Russia
}

\begin{abstract}
This paper presents a sequential estimation procedure for the unknown parameters of a continuous-time stochastic linear regression process. As examples the sequential estimation problem of two dynamic parameters in stochastic linear systems with memory and in autoregressive processes is solved. The estimation procedure is based on the least squares method with weights and yields estimators with guaranteed accuracy in the sense of the $L_{q}$-norm for fixed $q \geq 2$.

The proposed procedure works in the mentioned examples for all possible values of unknown dynamic parameters on the plane $R^{2}$ for the autoregressive processes and on the plane $R^{2}$ with the exception of some lines for the linear stochastic delay equations. The asymptotic behavior of the duration of observations is determined.

The general estimation procedure is designed for two- or more-parametric models. It is shown, that the proposed procedure can be applied to the sequential parameter estimation problem of affine stochastic delay differential equations and autoregressive processes of an arbitrary order.
\end{abstract}

AMS classification: $34 \mathrm{~K} 50 ; 60 \mathrm{H} 10 ; 62 \mathrm{~L} 10 ; 62 \mathrm{~L} 12$

Keywords and Phrases: Delay analysis; differential equations; estimation parameters; sequential identification; memory applications

\footnotetext{
1 The research on this paper was supported by RFBR - DFG 05-01-04004 Grant

${ }^{2}$ Result was presented at the IFAC WC 2008
} 


\section{Introduction}

In this article we consider a linear regression model of the type

$$
d x(t)=\vartheta^{\prime} a(t) d t+d W(t), t \geq 0
$$

with the initial condition $x(0)=x_{0}$. Here we assume $(W(t), t \geq 0)$ is an adapted one-dimensional standard Wiener process on a filtered probability space $(\Omega, F$, $\left.\left(F_{t}\right)_{t \geq 0}, P\right), \vartheta$ an unknown parameter from some subset $\Theta$ of $R^{p+1},(a(t), t \geq 0)$ an observable adapted $(p+1)$-dimensional cadlag process and $x=(x(t), t \geq 0)$ solves the equation (1). We assume $p>1$.

The described model includes several more concrete cases like linear stochastic differential equations of first or of higher order (CARMA-processes) linear stochastic delay differential equations. They can be found e.g. in [2], [3], [6], [7], [9]-[13], [16].

In the sequel we will study the problem of sequential estimating the parameter $\vartheta$ from $\Theta$ based on the observation of $(x(t), a(t))_{t \geq 0}$.

We shall construct for every $\varepsilon>0$ and arbitrary but fixed $q \geq 2$ a sequential procedure $\vartheta(\varepsilon)$ to estimate $\vartheta$ with $\varepsilon$-accuracy in the sense

$$
\|\vartheta(\varepsilon)-\vartheta\|_{q}^{2} \leq \varepsilon
$$

Here the $L_{q}-$ norm is defined as $\|\cdot\|_{q}=\left(E_{\vartheta}\|\cdot\|^{q}\right)^{\frac{1}{q}}$, where $\|a\|=\left(\sum_{i=0}^{m} a_{i}^{2}\right)^{\frac{1}{2}}$ and $E_{\vartheta}$ denotes the expectation under $P_{\vartheta}$ for $\vartheta \in \Theta$ (the number of $q \geq 2$ is fixed in the sequel).

Moreover, we shall determine the rate of convergence of the duration of observations $T(\varepsilon)$ to infinity and almost surely convergence of $\vartheta(\varepsilon)$ if $\varepsilon \rightarrow 0$.

The new results presented here consist in the greater generality of the conditions on $a(t)$ than in previous papers of [9]-[12]. A similar estimation problem for a more general model was investigated in [3]. The authors have considered the problem of sequential estimation of parameters in multivariate stochastic regression models with martingale noise and an arbitrary finite number of unknown parameters. The estimation procedure in [3] is based on the least squares method with a special choice of weight matrices. The proposed procedure enables them to estimate the parameters with any prescribed mean square accuracy under appropriate conditions on the regressors $(a(t))$. Among conditions on the regressors there is one limiting the growth of the maximum eigenvalue of the symmetric design matrix with respect to its minimal eigenvalue. This condition is slightly stronger than those usually imposed in asymptotic investigations and it is not possible to apply this estimation procedure to continuous-time models with essentially different behaviour of the eigenvalues (if, for example, the smallest eigenvalue growth linearly and the largest one - exponentially with the observation time).

The paper [3] also includes extended hints to earlier works of different authors on sequential estimations for parameters of both continuous as well as discrete time processes.

The methods applied in this paper to (1) were inspired by the following basic examples for (1): 
I. Stochastic differential equations of autoregressive type given by

$$
\begin{gathered}
d x_{t}^{(p)}=\sum_{i=0}^{p} \vartheta_{i} x_{t}^{(p-i)} d t+d W(t), t \geq 0, \\
x_{0}^{(p-i)}=x^{(p-i)}(0), i=\overline{0, p} .
\end{gathered}
$$

II. Stochastic delay differential equations given by

$$
\begin{gathered}
d X(t)=\sum_{i=0}^{p} \vartheta_{i} X\left(t-r_{i}\right) d t+d W(t), t \geq 0, \\
X(s)=X_{0}(s), s \in[-r, 0] .
\end{gathered}
$$

The parameters $\vartheta_{i}, r_{i}, i=0, \ldots, p$ are real numbers with $0=r_{0}<r_{1}<\ldots<$ $r_{p}=: r$, if $p \geq 1$ and $r_{0}=r=0$ if $p=0$. The initial process $\left(X_{0}(s), s \in[-r, 0]\right)$ also defined on $(\Omega, F, P)$ is supposed to be cadlag and all $X_{0}(s), s \in[-r, 0]$ and $x_{0}^{(p-i)}, i=\overline{0, p}$ are assumed to be $F_{0}$-measurable. Moreover it is assumed that

$$
E\left|x_{0}^{(p-i)}\right|^{q}<\infty, \quad i=\overline{0, p}, \quad E \int_{-r}^{0}\left|X_{0}(s)\right|^{q} d s<\infty .
$$

The sequential parameter estimation problem of the process (3) was solved in [7] under some additional condition on the roots of its characteristic equation (and as follows, on the corresponding parameters). Similar to [3], in [7] obtained the sequential estimators of the parameter $\vartheta$ with given accuracy in the mean square sense.

Our paper considers the sequential parameter estimation problem of the process (3) with $p=1$ as an example of the general estimation procedure, elaborated for linear regression model (1). It is shown, that the presented sequential estimation procedure works for all parameters $\vartheta \in R^{2} \backslash\left\{\vartheta \in R^{2}: \vartheta_{1}=0\right\}$. The asymptotic behaviour of the estimation procedures is investigated.

The problem of sequential parameter estimation for the process (4) was considered in [9]-[12] under some additional conditions on the underlying parameters. The general estimation procedure, presented in this paper, works under the most weakest possible assumptions on the parameters. Thus it is shown, that in the case $p=1$ in the model (4) the constructed general estimation procedure gives the possibility to solve the parameter estimation problem with guaranteed accuracy for all parameter points $\vartheta \in R^{2}$ except for some curves Lebesgue of measure zero.

The estimators with such property may be used in various adaptive procedures (control, prediction, filtration).

\section{The general case of regression process}

\subsection{Assumptions and definitions}

In this section we shall consider the linear regression model (1)

$$
d x(t)=\vartheta^{\prime} a(t) d t+d W(t), t \geq 0 .
$$


The problem is to estimate the unknown vector $\vartheta$ with a given accuracy in the sense (2) from the observation of $(x(t), a(t))_{t \geq 0}$.

The differential equation (3) is covered by $a(t)=\left(x_{t}^{(p)}, x_{t}^{(p-1)}, \ldots, x_{t}\right)^{\prime}$ and the equation (4) by $a(t)=\left(X(t), X\left(t-r_{1}\right), \ldots, X\left(t-r_{p}\right)\right)^{\prime}$.

In Sections 3 and 4 we shall consider the models (3) and (4) in detail.

A natural candidate for estimating $\vartheta$ is the least squares estimator (LSE)

$$
\tilde{\vartheta}(T)=\left(\int_{0}^{T} a(t) a^{\prime}(t) d t\right)^{-1} \int_{0}^{T} a(t) d x(t), \quad T>0 .
$$

It turns out in examples that the information matrix $\int_{0}^{T} a(t) a^{\prime}(t) d t$ has different asymptotic properties for different parameters $\vartheta$. Thus e.g., the information matrix normalized by a scalar function may tend to a singular limit matrix.

To avoid this problem we rewrite the expression of the LSE $\tilde{\vartheta}(T)$ above in such a way, that as the inverse matrix factor there appears an appropriate chosen normalized matrix for which the asymptotic behaviour of its maximal eigenvalue for $T \rightarrow \infty$ is under control. To do this we apply a certain matrix $V$ as a weight matrix to $a(t)$ to obtain the new process $(V a(t))$ with better asymptotic properties in the sense of Assumption (V) below (see formula (7)). The concrete form of $V$ is determined by the kind of regressor $a(t)$ and cannot specified for the general case. Moreover $V$ may depend on the unknown $\vartheta$. To overcome these problem we shall construct a process $(V(t))$ based on the observations of $(x, a)$ up to $t$, which estimates $V$ and keeps the property (7) for the observed process $(b(t))_{t \geq 0}$, where $b(t)=V(t) a(t)$.

To get a first estimation of $V$ by $V(\cdot)$ and some rates of convergence which defined below, we use the observation $(x, a)$ from 0 to some time $S$. The properly estimation of the parameter $\vartheta$ starts from $S$.

The weighted LSE of $\vartheta$ for the given observation from $S$ to $T$ has the form:

$$
\hat{\vartheta}(S, T)=G^{-1}(S, T) \Phi(S, T), T>S>0,
$$

where

$$
\Phi(S, T)=\int_{S}^{T} b(s) d x(s), G(S, T)=\int_{S}^{T} b(s) a^{\prime}(s) d s,
$$

$b(s)=V(s) a(s)$. Put $\Phi(T)=\Phi(0, T), \quad G(T)=G(0, T), \quad \bar{b}(s)=V a(s)$.

Let the weight process $(V(t))_{t \geq 0}$ be $\left(F_{t}\right)$-adapted and for all $T>0$ the following integrals be finite:

$$
\int_{0}^{T} E_{\vartheta}\|b(t)\|^{q} d t<\infty .
$$

We shall write in the following $f(x) \simeq C$ as $x \rightarrow \infty\left(f_{\varepsilon} \simeq C\right.$ as $\left.\varepsilon \rightarrow 0 \ldots\right)$ instead of the limiting relations:

$$
0<\lim _{x \rightarrow \infty} f(x) \leq \varlimsup_{x \rightarrow \infty} f(x)<\infty \quad\left(0<\varliminf_{\varepsilon \rightarrow 0} f_{\varepsilon} \leq \varlimsup_{\varepsilon \rightarrow 0} f_{\varepsilon}<\infty \ldots\right) .
$$


The rates of increase of the integrals $\int_{0}^{T} b_{i}^{2}(t) d t, i=\overline{0, p}$ in general depend on some vector parameter $\alpha \in R^{r}$.

$\operatorname{ASSUMPTION~}(V)$ : Let $\mathcal{A}$ be a non-empty subset of $R^{r}$, such that, for every $i=\overline{0, p}$ there exists a family of unboundedly increasing positive functions $\left\{\varphi_{i}(\alpha, T), T>\right.$ $0\}_{\alpha \in \mathcal{A}}$ with the following properties: for every $\vartheta \in \Theta$ and $\alpha(\vartheta) \in \mathcal{A}$

$$
\varphi_{i}^{-1}(\alpha, T) \int_{0}^{T} \tilde{b}_{i}^{2}(t) d t \simeq C, \quad \text { as } \quad T \rightarrow \infty \quad P_{\vartheta}-\text { a.s. }
$$

where $\tilde{b}_{i}(\cdot)$ equals $b_{i}(\cdot)$ or $\bar{b}_{i}(\cdot), i=\overline{0, p}$.

For example, the function $\varphi(\alpha, T)=T^{v_{1}} \mathrm{e}^{v_{0} T}$, where

$$
A=\left\{\left(v_{0}, v_{1}\right):[\{0\} \times(0,+\infty)] \cup[(0,+\infty) \times(-\infty,+\infty)]\right\}
$$

cover all possible cases of asymptotic behavior of solutions of linear SDE's and SDDE's (see our main examples below).

Often we shall omit the dependence $\varphi_{i}(\alpha, T)$ of the parameter $\alpha$ in our notation. The functions $\varphi_{i}(\alpha, T)$ are called rates of increase of integrals $\int_{0}^{T} b_{i}^{2}(t) d t$ and $\int_{0}^{T} \bar{b}_{i}^{2}(t) d t \quad i=\overline{0, p}$.

Our sequential plans will be constructed by using first hitting times of the processes $\int_{0}^{T} b_{i}^{2}(s) d s, i=\overline{0, p}, T>0$. To investigate the asymptotic properties of these hitting times, we will use the rates $\varphi_{i}(T)$ of increase of these integrals.

Without loss of generality we suppose in Assumption (V), that the function $\varphi_{0}(\alpha, T)$ is the smallest rate of increase in the following sense:

$$
\varlimsup_{T \rightarrow \infty} \frac{\varphi_{0}(\alpha, T)}{\varphi_{i}(\alpha, T)} \leq 1, \quad i=\overline{1, p} .
$$

Otherwise we shall renumber the lines in the weight matrices $V, V(T)$ to obtain this property.

In Sections 3 and 4 we will get the weights $V,(V(t))$ and the rates $\left(\varphi_{i}(\alpha, T), i=\right.$ $\overline{0, p}$ ) for both our basic examples in the case $p=1$.

From (1) and (5) we find the deviation of the estimator $\hat{\vartheta}(S, T)$ from $\vartheta$ :

$$
\hat{\vartheta}(S, T)-\vartheta=G^{-1}(S, T) \zeta(S, T),
$$

where

$$
\zeta(S, T)=\int_{S}^{T} b(t) d W(t)
$$


Assumption (V) motivated by two our basic examples provide the asymptotic behavior of the integrated squares of the function $b(t)$. It should be noted that the second moment of the noise $\zeta(S, T)$ is a functional of $b(\cdot)$, which is assumed below to be controlled:

$$
E_{\vartheta}\|\zeta(S, T)\|^{2}=E_{\vartheta} \int_{S}^{T}\|b(t)\|^{2} d t .
$$

Our sequential plans will be constructed by using first hitting times of the processes $\int_{S}^{T} b_{i}^{2}(s) d s$.

To investigate the asymptotic properties of the estimator $\hat{\vartheta}(S, T)$, we introduce the matrices

$$
\begin{aligned}
& \bar{\varphi}(T)=\operatorname{diag}\left\{\varphi_{0}(\alpha, T), \varphi_{1}(\alpha, T), \ldots, \varphi_{p}(\alpha, T)\right\}, \quad \tilde{\varphi}^{\frac{1}{2}}(T)=\bar{\varphi}^{\frac{1}{2}}(T)\left(V^{\prime}\right)^{-1}, \\
& \bar{G}(S, T)=\bar{\varphi}^{-\frac{1}{2}}(T) G(S, T) \tilde{\varphi}^{-\frac{1}{2}}(T), \quad \tilde{G}(S, T)=\bar{\varphi}^{-\frac{1}{2}}(T) G(S, T) \varphi_{0}^{-\frac{1}{2}}(T), \\
& \bar{G}(T)=\bar{G}(0, T), \quad \tilde{G}(T)=\tilde{G}(0, T), \quad \bar{\zeta}(S, T)=\bar{\varphi}^{-\frac{1}{2}}(T) \int_{S}^{T} b(t) d W(t) .
\end{aligned}
$$

The reader can easily check, that for calculation of $\tilde{G}(S, T)$ the knowledge of $V$ (which is unknown) is not necessary, as it was for the calculation of $\bar{G}(S, T)$.

First we investigate the rate of convergence of the estimator $\hat{\vartheta}(S, T)$ using the following form of its normalized deviation from $\vartheta$ :

$$
\tilde{\varphi}^{\frac{1}{2}}(T)(\hat{\vartheta}(S, T)-\vartheta)=\bar{G}^{-1}(S, T) \bar{\zeta}(S, T) .
$$

As follows from our basic examples, the matrix $\bar{G}(T)$ may be get degenerated as $T \rightarrow \infty$ (see Table 1 , region $\tilde{\Theta}_{14}$ and Table 4 , region $\Theta_{13}^{*}$ ). In this case, the limit of $\tilde{\varphi}^{\frac{1}{2}}(T)(\hat{\vartheta}(S, T)-\vartheta)$ for $T \rightarrow \infty$ can be calculated if we know the rate of decreasing of the smallest eigenvalue of $\bar{G}^{\prime}(T) \bar{G}(T)$ for $T \rightarrow \infty$. The following Assumption (G) below gives this rate.

To formulate Assumption (G) we define the following sets of functions

$$
\begin{gathered}
P_{0}=\left\{f(\cdot): \frac{f(y(x))}{f(x)} \simeq C \text { if } \frac{y(x)}{x} \simeq C \text { as } x \rightarrow \infty\right\}, \\
\bar{P}_{0}=\left\{f(\cdot): \frac{y(x)}{x} \simeq C \text { if } \frac{f(y(x))}{f(x)} \simeq C \text { as } x \rightarrow \infty\right\}, \\
\mathcal{G}_{0}=\left\{g(\cdot) \in P_{0}: \frac{\lim }{T \rightarrow \infty} g(T)>0\right\}
\end{gathered}
$$

and for $g(\cdot) \in \mathcal{G}_{0}$ the sets

$$
\begin{gathered}
P_{1}(g)=\left\{y(\cdot): \text { such that } y(S)=o\left(\bar{g}^{-1 / 2}(T) y(T)\right) \text { if } S=o(T) \text { as } T \rightarrow \infty\right\} \\
\bar{P}_{1}(g)=\left\{y(\cdot): \text { such that } S=o(T) \text { if } y(S)=o\left(\bar{g}^{-1 / 2}(T) y(T)\right) \text { as } T \rightarrow \infty\right\} \\
\mathcal{G}_{1}=\left\{g(\cdot) \in \mathcal{G}_{0}: P_{1}(g) \neq \varnothing\right\}, \quad \overline{\mathcal{G}}_{1}=\left\{g(\cdot) \in \mathcal{G}_{0}: \bar{P}_{1}(g) \neq \varnothing\right\}
\end{gathered}
$$


Note, that the functions $g(\cdot)$ given in Tables 2 and 4 below belong (see our examples) to the sets $G_{1}$ and $\bar{G}_{1}$.

$\operatorname{ASSUMPTION~}(\mathrm{G})$ : Let the functions $\bar{b}(t)$ and $b(t)$ satisfy Assumption $(V)$ and let $\varphi_{i}(\cdot) \in P_{1}(g), i=\overline{0, p}, g(\cdot) \in \mathcal{G}_{1}$. We suppose that the following property for the matrix function $\bar{G}(T)$ and $\bar{g}(T)=g\left(\varphi_{0}(T)\right), g(\cdot) \in \mathcal{G}_{1}$ holds:

$$
\lim _{T \rightarrow \infty} \bar{g}(T) \lambda_{\min }\left\{\bar{G}^{\prime}(T) \bar{G}(T)\right\}>0 P_{\vartheta} \text { - a.s., }
$$

According to Assumptions $(\mathrm{V})$ and $(\mathrm{G})$, the variances of the components of the vector of noises $\bar{\zeta}(T)$ are asymptotically bounded from above and the matrix $\bar{g}^{-\frac{1}{2}}(T) \bar{G}^{-1}(S, T)$ is bounded $P_{\vartheta}-$ a.s. on the norm from above for all $S, T$ large enough with $T>S$. Then we can say that the components of the vector estimator $\hat{\vartheta}(S, T)$ have rates of convergence to the true value $\vartheta$ equals to the corresponding diagonal elements of the matrix $\bar{g}^{-\frac{1}{2}}(T) \tilde{\varphi}^{\frac{1}{2}}(T)$.

Consider two extreme cases. If $V=I$ then the estimator $\hat{\vartheta}$ has the fastest rate of convergence $\bar{g}^{-\frac{1}{2}}(T) \tilde{\varphi}^{1 / 2}(T)=\bar{g}^{-\frac{1}{2}}(T) \bar{\varphi}^{\frac{1}{2}}(T)$. If, on the contrary, the matrix $V$ has more complicate structure, then the rates of convergence of all the components of the vector estimator $\hat{\vartheta}(S, T)$ may proportional to the slowest rate $\bar{g}^{-\frac{1}{2}}(T) \varphi_{0}^{\frac{1}{2}}(T)$.

Our purpose is to consider the most general case of non-constant weights $V(t)$ with an unknown non-degenerate limit matrix $V$ of an arbitrary structure (according to planned applications). Therefore we shall use the following normalized representation for the deviation of the $\operatorname{LSE} \hat{\vartheta}(S, T)$ :

$$
\varphi_{0}^{\frac{1}{2}}(T)(\hat{\vartheta}(S, T)-\vartheta)=\tilde{G}^{-1}(S, T) \bar{\zeta}(S, T),
$$

where we use the matrix $\tilde{G}(S, T)$, which does not depends from the unknown matrix $V$ (in contrast to the matrix $\bar{G}(S, T)$ ). At the same time, as we show below, the matrices $\tilde{G}(S, T)$ and $\bar{G}(S, T)$ have similar asymptotic properties under following assumption and condition (12) (see below).

We will use in the sequel the notation $T: S \uparrow \infty$ for $S=o(T), T \rightarrow \infty$.

Assumption (G) is more convenient for verification for the matrix $\bar{G}(\cdot)$ than for the matrix $\tilde{G}(\cdot)$. At the same time it gives the possibility to control the behaviour of the matrix $\tilde{G}^{-1}(S, T)$ in the representation (9) of the deviation of the estimator $\hat{\vartheta}(S, T)$ from $\vartheta$ by the construction of sequential estimation plans.

This is true in view of the following inequalities for the norm $\left\|\tilde{G}^{-1}(S, T)\right\|^{2}$, obtained in Proposition 1 (see Appendix):

$$
\varlimsup_{T: S \uparrow \infty} \bar{g}^{-1}(T)\left\|\tilde{G}^{-1}(S, T)\right\|^{2}<\infty
$$

and the lower limiting bound

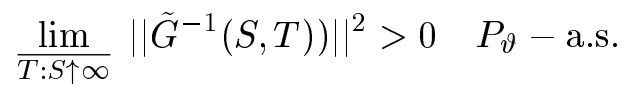

can be obtained under the following additional condition on the functions $\varphi_{i}(T), i=$ $\overline{0, p}$ and on the matrix $V$ :

$$
\lim _{T \rightarrow \infty} \lambda_{\max }\left\{V^{\prime} \bar{\varphi}^{-1}(T) \varphi_{0}(T) V\right\}>0 .
$$


By the definition, the noise $\bar{\zeta}(S, T)$ is bounded from above in the $L_{q}-$ norm. Thus, according to $(9)$ and (10) we can say that the estimator $\hat{\vartheta}(S, T)$ has the rate of convergence $\bar{g}^{-1 / 2}(T) \varphi_{0}^{\frac{1}{2}}(T)$ and, as follows, it is oriented on the at most "bad case" (on the second of the mentioned just before (9) extreme cases). This is the payment for the lower level of a'priori information on the observed process $(a(t))$.

Now we introduce different parametric classes for the functions $\varphi_{i}(\alpha, T)$ which reflect, in particular, all possible cases of asymptotic behavior of solutions of linear stochastic differential equations (SDE's) and stochastic delay differential equations (SDDE's).

In the sequel we say that functions $f$ and $g$ are equivalent asymptotically for $T$ large enough $(f(T) \sim g(T))$ if $f(T) / g(T) \rightarrow C$ as $T \rightarrow \infty$ for some positive number $C$.

ASSUMPTION $(\varphi \Psi)$ : Assume $\varphi_{i}(\alpha, T), i=\overline{0, p}, \alpha \in \mathcal{A}$ are functions as described in Assumption $(V)$. We put $\Psi_{0}(\alpha, x)=x$ and suppose, that there exist so-called positive rate generating functions $\Psi_{i}(\cdot, \cdot), i=\overline{1, p}$ on $\mathcal{A} \times(0, \infty)$, such that $\varphi_{i}(\alpha, T) \sim \Psi_{i}\left(\alpha, \varphi_{0}(T)\right), i=\overline{1, p}$ for all $\alpha \in \mathcal{A}$.

To formulate the forthcoming assumptions we need some special classes of just introduced rate generating functions $\Psi_{i}(\alpha, T)$ which we shall define in following

DEFINITION (D1). For every vector $\bar{i}_{k}=\left(i_{1}, \ldots, i_{k}\right)$ of increasing integers $i_{j} \in$ $[0, p], j=\overline{1, k}, k=\overline{1, p+1}$ and fixed $\alpha \in \mathcal{A}$ as well as for every vector of rate generating functions $\Psi\left[\alpha, \bar{i}_{k}\right]:=\left(\Psi_{i_{1}}(\alpha, x), \ldots, \Psi_{i_{k}}(\alpha, x)\right)$, we define $Y\left(\Psi\left[\alpha, \bar{i}_{k}\right]\right)$ to be the set of all real functions $y(\cdot)$ on $(0, \infty)$ such that

$$
\frac{\Psi_{i_{1}}(\alpha, y(x))}{\Psi_{i_{1}}(\alpha, x)}+\ldots+\frac{\Psi_{i_{k}}(\alpha, y(x))}{\Psi_{i_{k}}(\alpha, x)} \simeq C \text { as } x \rightarrow \infty
$$

and $Y^{\prime}\left(\Psi\left[\alpha, \bar{i}_{k}\right]\right)$ to be the set of all real functions $y(\cdot)$ on $(0, \infty)$ with the property

$$
\frac{\Psi_{i_{1}}(\alpha, x)}{\Psi_{i_{1}}(\alpha, y(x))}+\ldots+\frac{\Psi_{i_{k}}(\alpha, x)}{\Psi_{i_{k}}(\alpha, y(x))} \simeq C \text { as } x \rightarrow \infty
$$

For every $k=\overline{1, p+1}$ and $\bar{i}_{k}=\left(i_{1}, \ldots, i_{k}\right), \alpha \in \mathcal{A}$ we define

$$
P_{k}(\alpha):=\left\{\Psi\left[\alpha, \bar{i}_{k}\right]: \quad Y\left(\Psi\left[\alpha, \bar{i}_{k}\right]\right) \subseteq Y^{\prime}\left(\Psi\left[\alpha, \bar{i}_{k}\right]\right)\right\} .
$$

We say that the functions $\varphi_{i_{1}}(T), \ldots, \varphi_{i_{k}}(T)$ are $P_{k}(\alpha)$-equivalent if their rate generating functions $\Psi_{i_{1}}(\alpha, x), \ldots, \Psi_{i_{k}}(\alpha, x)$ are components of some vector $\Psi\left[\alpha, \bar{i}_{k}\right] \in$ $P_{k}(\alpha)$.

Fix a certain $\alpha \in \mathcal{A}$. In some sense one could say, that the set $P_{k}(\alpha)$ consists of vectors of functions, the increase rates of which differ not essentially. For example, $\Psi\left[\alpha, \bar{i}_{2}\right]=\left(x, x^{\alpha}\right) \in P_{2}(\alpha)$ for every $\alpha \in R^{1}, \Psi\left[\alpha, \bar{i}_{2}\right]=\left(\mathrm{e}^{2 \alpha_{0} x}, \mathrm{e}^{2 \alpha_{1} x}\right) \in P_{2}(\alpha)$ for $\alpha=\left(\alpha_{0}, \alpha_{1}\right), \alpha_{0}>0, \alpha_{1}>0$ and $\Psi\left[\alpha, \bar{i}_{2}\right]=\left(x, \mathrm{e}^{2 \alpha x}\right) \notin P_{2}(\alpha)$ for $\alpha>0$.

Let $S$ and $T$ be two reals with $0 \leq S<T$. The part of observations $(x(s), a(s), 0 \leq$ 
$s \leq S)$ will be used to estimate $\alpha$, the part $(x(t), a(t), S \leq t \leq T)$ to estimate $\vartheta$. The problem of estimation $\alpha$ will be observed in the next point 2.2. Now we consider the problem of estimation $\vartheta$.

Our aim is to construct sequential plans for estimating the parameters $\vartheta_{i}, i=\overline{0, p}$. This will be done below by using the processes $\int_{S}^{T} b_{i}^{2}(t) d t, i=\overline{0, p}$. The rate of increase of these processes is connected with the behavior of $\varphi_{i}(T)$ for $T \rightarrow \infty$ and may be different for different $i$, see Assumption (V). Similar to our previous papers, we will construct stopping times based on the sums of the integrals $\int_{S}^{T} b_{i}^{2}(t) d t, i=\overline{0, p}$. In the case, when the rates of increase of these integrals may differ essentially, we can not derive asymptotic properties of these stopping times. Thus we shall construct different systems of stopping times on the basis of these processes (which are by the way the quadratic variations of the martingales $\left.\zeta_{i}(S, T), i=\overline{0, p}\right)$ to control the moments of the noise $\zeta(S, T)$.

Our following purpose is to divide the set of functions $\varphi_{0}(T), \varphi_{1}(T), \ldots, \varphi_{p}(T)$ into some groups of size $l_{i}$ say, such that the rates of increase of these functions do not differ essentially within. To this aim we introduce some notation. Let

$$
I_{p}:=\left\{\bar{i}_{k}=\left(i_{1}, \ldots, i_{k}\right): 0 \leq i_{1}<i_{2}<\ldots<i_{k} \leq p, \quad k=\overline{1, p+1}\right\}
$$

be the set of all the vectors of indexes of the dimension less or equal $p+1$.

Choose recurrently a sequences of numbers $l_{r}$ and vectors $\bar{j}^{r}$ as follows: $l_{-1}=-1$,

$$
l_{0}:=\max \left\{k=\overline{1, p+1}: \Psi\left[\alpha, \bar{i}_{k}\right] \in P_{k}(\alpha), \bar{i}_{k} \in I_{p}, i_{1}=0\right\},
$$

$\bar{j}^{0}$ is the corresponding vector, satisfying $\Psi\left[\alpha, \bar{j}^{0}\right] \in P_{l_{0}}(\alpha)$;

Denote $s_{j}=\sum_{i=-1}^{j} l_{i}, j \geq-1$. For $r \geq 1$ we define

$$
l_{r}:=\max \left\{k=\overline{1, p-s_{r-1}}: \Psi\left[\alpha, \bar{i}_{k}\right] \in P_{k}(\alpha), \quad \bar{i}_{k} \in I_{p} \backslash\left(\cup_{i=0}^{r-1} \bar{j}^{i}\right)\right\}
$$

if $s_{r-1}<p$ and 0 otherwise; $\bar{j}^{r}$ is one of the vectors $\bar{i}_{l_{r}}$, satisfying the relation $\Psi\left[\alpha, \bar{i}_{l_{r}}\right] \in P_{l_{r}}(\alpha)$ and having the smallest first component.

Put

$$
m:=\min \left\{j \geq 0: s_{j}=p\right\} .
$$

It is obviously, that $0 \leq m \leq p$.

Thus we have defined the lengths $l_{i}, i=\overline{0, m}$ of mentioned above groups of functions. Then we unify all the functions $\varphi_{0}(T), \varphi_{1}(T), \ldots, \varphi_{p}(T)$ in $m+1$ groups $G_{j}=[\cdot, \ldots, \cdot]$ of $P_{l_{j}}(\alpha)$-equivalent functions respectively, $j=\overline{0, m}$, and without loss of generality can introduce, for simplification of our notation, the ordering of these groups in such a way that $G_{i}=\left[\varphi_{s_{i-1}+1}(T), \ldots, \varphi_{s_{i}}(T)\right], i=\overline{0, m}$ (it can be achieved by permutation of the lines in the weight matrix $V$ ).

Consider one simple example to explain the introduced notation. Assume we have five $(p=4)$ functions, defined as follows:

$$
\varphi_{0}(T)=T, \varphi_{1}(T)=\mathrm{e}^{\alpha_{1} T}, \varphi_{2}(T)=\mathrm{e}^{\left(\mathrm{e}^{\alpha} 2^{T}\right)},
$$




$$
\varphi_{3}(T)=T, \varphi_{4}(T)=\mathrm{e}^{\alpha_{3} T}, \alpha_{i}>0, i=\overline{1,3} .
$$

Then $\alpha=\left(\alpha_{1}, \alpha_{2}, \alpha_{3}\right)$, the functions $\varphi_{0}(T)$ and $\varphi_{3}(T)$ are $P_{2}(\alpha)$-equivalent, the functions $\varphi_{1}(T)$ and $\varphi_{4}(T)$ are $P_{2}(\alpha)$-equivalent and we can find the vectors:

$$
\bar{j}^{0}=\{(0,3)\}, \quad \bar{j}^{1}=\{(1,4)\}, \bar{j}^{2}=\{(2)\} .
$$

Then $l_{0}=2, l_{1}=2, l_{2}=1, m=2$ and we obtain three groups of functions:

$$
G_{1}=\{T, T\}, G_{2}=\left\{\mathrm{e}^{\alpha_{1} T}, \mathrm{e}^{\alpha_{3} T}\right\}, G_{3}=\left\{\mathrm{e}^{\left(\mathrm{e}^{\alpha_{2} T}\right)}\right\} .
$$

We give now an additional assumption on the functions $\Psi_{i}(\cdot, \cdot), i=\overline{0, p}$ for the case $m>0$.

$\operatorname{ASSUMPTION}(\Psi):$ Assume $\Psi_{i}(\alpha, x), i=\overline{0, p}, \alpha \in \mathcal{A}$ are the functions from Assumption $(\varphi \Psi)$ and $m>0$. We suppose, that there exist some integers $i_{k} \in\left[s_{j-1}+\right.$ $\left.1, s_{j}\right]$ for every $j=\overline{0, m}$ such that the functions $\Psi_{i_{k}}(\alpha, \cdot) \in \bar{P}_{0}$ and $\varphi_{0}(\cdot) \in \bar{P}_{1}(g)$, $g \in \overline{\mathcal{G}}_{1}$.

For example, the function $\Psi(\alpha, x)=x^{v_{1}} \mathrm{e}^{v_{0} x}$ belongs to the class $\bar{P}_{0}$ if

$$
A=\left\{\left(v_{0}, v_{1}\right):[\{0\} \times(0,+\infty)] \cup[(0,+\infty) \times(-\infty,+\infty)]\right\} .
$$

By the construction of our sequential plans we shall define $m+1$ systems of stopping times on the bases of the sums of appropriately normalized integrals $\int_{S}^{T} b_{i}^{2}(t) d t$, having the rates of increase $\varphi_{i}(T), i=\overline{s_{j-1}+1, s_{j}}$ with the rate generating functions from the corresponding groups $G_{j}, j=\overline{0, m}$.

To take this aim into account we introduce a "multidimensional time scale" $\bar{T}=(\underbrace{T_{0}, \ldots, T_{0}}_{l_{0}}, \underbrace{T_{1}, \ldots, T_{1}}_{l_{1}}, \ldots, \underbrace{T_{m}, \ldots, T_{m}}_{l_{m}})$ if $m>0, \bar{T}=(\underbrace{T_{0}, \ldots, T_{0}}_{p+1})$ if $m=0$. We shall substitute in the following the components of the vector $\bar{T}$ on the special stopping times.

Denote $T_{\max }=\max _{i=\overline{0, m}} T_{i}$ and $T_{\min }=\min _{i=\overline{0, m}} T_{i}$. We shall construct our sequential estimation plans on the bases of the estimator $\hat{\vartheta}(S, T)$ with $T=T_{m i n}$, which has the rate of convergence equals to $\bar{g}^{-\frac{1}{2}}(T) \varphi_{0}^{\frac{1}{2}}(T)$ as $T \rightarrow \infty$. At the same time we will use for estimation the sample of the size $T_{\max }$. To keep the order of the convergence rate $\bar{g}^{-\frac{1}{2}}\left(T_{\min }\right) \varphi_{0}^{\frac{1}{2}}\left(T_{m i n}\right)$ it is natural to demand the following property:

$$
\varlimsup_{\bar{T} \rightarrow \infty} \frac{\bar{g}^{-\frac{1}{2}}\left(T_{\max }\right) \varphi_{0}^{\frac{1}{2}}\left(T_{\max }\right)}{\bar{g}^{-\frac{1}{2}}\left(T_{\min }\right) \varphi_{0}^{\frac{1}{2}}\left(T_{\min }\right)}<\infty .
$$

In view of the definition of the function $\bar{g}(T), g \in \mathcal{G}_{0}$, this relation holds true on the following admissible set for the time-scales $\bar{T}$ :

$$
\Upsilon:=\left\{(\bar{T}): \varlimsup_{\bar{T} \rightarrow \infty} \varphi_{0}\left(T_{\max }\right) / \varphi_{0}\left(T_{\min }\right)<\infty\right\} .
$$




\subsection{Construction of sequential estimation plans}

Let us return to the study of the equation (1) and assume that the Assumptions $(\mathrm{V}),(\mathrm{G}),(\varphi \Psi)$ and $(\Psi)$ are valid.

Let $\varepsilon$ be any positive number being fixed in the sequel. Now we construct a sequential estimation plan $\operatorname{SEP}(\varepsilon)=\left(T(\varepsilon), \vartheta_{\varepsilon}^{*}\right)$ where $T(\varepsilon)$ and $\vartheta_{\varepsilon}^{*}$ are the duration of estimation and the estimator of $\vartheta$ with the $\varepsilon$-accuracy in the sense of $L_{q}$-norm (2) respectively.

To construct a sequential estimator $\vartheta(\varepsilon)$ of $\vartheta$ with preassigned accuracy $\varepsilon$ first we introduce a random time substitution for the weighted least square estimator $\hat{\vartheta}(S, T)$ from (5). This enables us to control the moments of the process $\bar{\zeta}(S, T)$ in the representation (9) of its deviation. To do that, we have to take into account the fact, that the $L_{q}$-norms of the components of the vector $b$ may have different rates of increasing. The knowledge of these rates gives the possibility to construct the system of stopping times belonging to the admissible set $\Upsilon$.

For every positive $\varepsilon$ let us fix two unboundedly increasing sequences $\left(\nu_{n}(\varepsilon)\right)_{n \geq 1}$ and $\left(c_{n}\right)_{n \geq 1}$ of positive $\left(F_{t}\right)$-adapted stopping times (or real numbers) and real numbers respectively, satisfying the following conditions: as $n \rightarrow \infty$ and/or $\varepsilon \rightarrow 0$

$$
\begin{gathered}
\varphi_{0}\left(\nu_{n}(\varepsilon)\right)=o\left(g^{-1 / 2}\left(\varepsilon^{-1} c_{n}\right) \varepsilon^{-1} c_{n}\right) P_{\vartheta}-\text { a.s. } \\
\sum_{n \geq 1} c_{n}^{-q / 2}<\infty
\end{gathered}
$$

and for every fixed $\varepsilon>0$

$$
\sum_{n \geq 1} g^{-q / 2}\left(\varepsilon^{-1} c_{n}\right)=\infty
$$

where $\bar{g}(T)=g\left(\varphi_{0}(T)\right), g(\cdot) \in \overline{\mathcal{G}}_{1}$.

Assume that $\alpha$ is a parameter of the functions $\varphi_{i}(\alpha, T), i=\overline{0, p}$ from Definition (D1), which can be estimated consistently by observation of $(x(t), a(t))_{t \geq 0}$. It is the case in all of our examples below.

Denote by $\alpha_{i}(n, \varepsilon), i=\overline{1, r}, n \geq 1$ some estimators of the parameters $\alpha_{i}, i=\overline{1, r}$, which we assume to be constructed using the trajectory of the observation process $(x, a)$ of the duration $\nu_{n}(\varepsilon)$. Define

$$
\begin{gathered}
\Psi(\alpha, n, \varepsilon)=\operatorname{diag}\left\{\varepsilon^{-1} c_{n}, \Psi_{1}\left(\alpha, \varepsilon^{-1} c_{n}\right), \ldots, \Psi_{p}\left(\alpha, \varepsilon^{-1} c_{n}\right)\right\}, \\
\tilde{\Psi}(n, \varepsilon)=\Psi(\alpha(n, \varepsilon), n, \varepsilon), \quad \tilde{b}_{n}(t)=\tilde{\Psi}^{-1 / 2}(n, \varepsilon) b(t)=\left(\tilde{b}_{0 n}(t), \ldots, \tilde{b}_{p n}(t)\right)^{\prime} .
\end{gathered}
$$

$\operatorname{ASSUMPTION}(\alpha)$ : Let the condition (14) be fulfilled. The estimators $\alpha(n, \varepsilon)$ of the parameter $\alpha$ are supposed to have the properties:

$\operatorname{ASSUMPTION}(\alpha 1)$ : for every $\varepsilon>0$ and $i=\overline{1, p}$

$$
\frac{\tilde{\Psi}_{i i}(n, \varepsilon)}{\Psi_{i i}(\alpha, n, \varepsilon)} \simeq C \text { as } n \rightarrow \infty \quad P_{\vartheta}-\text { a.s. }
$$


$\operatorname{ASSUMPTION}(\alpha 2)$ : for every $n \geq 1$ and $i=\overline{1, p}$

$$
\frac{\tilde{\Psi}_{i i}(n, \varepsilon)}{\Psi_{i i}(\alpha, n, \varepsilon)} \simeq C \text { as } \varepsilon \rightarrow 0 \quad P_{\vartheta}-\text { a.s. }
$$

In Section 3 Assumption $(\alpha)$ will be verified for the autoregressive process (3), considered in Example I and in Section 4 for the time delayed process (4) from Example II.

Let us define the sequences of stopping times $\left(\tau_{j}(n, \varepsilon), n \geq 1\right), j=\overline{0, m}$ as follows

$$
\tau_{j}(n, \varepsilon)=\inf \left\{T>\nu_{n}(\varepsilon): \sum_{i=s_{j-1}+1}^{s_{j}}\left(\int_{\nu_{n}(\varepsilon)}^{T} \tilde{b}_{i n}^{2}(t) d t\right)^{q / 2}=1\right\}
$$

where $\inf \{\oslash\}=\infty$ and denote

$$
\tau_{\min }(n, \varepsilon)=\min \left\{\tau_{0}(n, \varepsilon), \tau_{1}(n, \varepsilon), \ldots, \tau_{m}(n, \varepsilon)\right\} .
$$

Note, that for $q=2$ and $m=0$ the definition (17) can be written in the form

$$
\tau_{0}(n, \varepsilon)=\inf \left\{T>\nu_{n}(\varepsilon): \int_{\nu_{n}(\varepsilon)}^{T}\left\|\tilde{b}_{n}(t)\right\|^{2} d t=1\right\} .
$$

Moreover, in the case $\varphi_{0}(T)=\ldots=\varphi_{p}(T)$ we can put $V(t) \equiv I, \nu_{n}(\varepsilon) \equiv 0$ and

$$
\tau_{0}(n, \varepsilon)=\inf \left\{T>0: \operatorname{tr} G(T)=\varepsilon^{-1} c_{n}\right\}
$$

(see, for comparison, [9]).

All these stopping times are finite and tend to infinity $P_{\vartheta}-$ a.s. if $n \rightarrow \infty$ or $\varepsilon \rightarrow 0$ due to the Assumption (V). The stopping times $\tau_{j}(n, \varepsilon)$, are constructed by using different sequences $\left(\tilde{\Psi}_{i}(n, \varepsilon), n \geq 1\right), i=\overline{s_{j-1}+1, s_{j}}, j=\overline{0, m}$, because, according to the Assumption (V), the rates of increase of functions $\int_{0}^{T} b_{i}^{2}(t) d t$ from different groups are different essentially.

From the condition (6), the definition (8) of the martingales $\zeta_{i}(S, T)$ and the Burkholder-Gundy inequality it follows that for any $q \geq 2$ the sequences

$$
\left(\zeta_{i}\left(\nu_{n}(\varepsilon), \tau_{j}(n, \varepsilon)\right), n \geq 1\right), i=\overline{s_{j-1}+1, s_{j}}, j=\overline{0, m}
$$

satisfy for $n \geq 1$ the inequalities

$$
E_{\vartheta} \zeta_{i}^{q}\left(\nu_{n}(\varepsilon), \tau_{j}(n, \varepsilon)\right) \leq b_{q} E_{\vartheta}\left(\int_{\nu_{n}(\varepsilon)}^{\tau_{j}(n, \varepsilon)} b_{i}^{2}(t) d t\right)^{q / 2}
$$

where $b_{q}$ is some positive constant. The value of $b_{q}$ can be obtained by making use of inequalities for local martingales (see Theorem 7 of Chapter 1 in [17] and [11]):

$$
b_{q}=2^{q-1}\left[3^{q-1}+2^{\frac{q}{2}}\left(1+q^{q}\right)\right]\left[\frac{q+1}{(q-1)^{q-1}}\right]^{\frac{q}{2}}
$$


for $q>2$ and $b_{2}=1$.

As follows, for the vector of noises

$$
\zeta_{n, \varepsilon}=\tilde{\Psi}^{-1 / 2}(n, \varepsilon) \zeta\left(\nu_{n}(\varepsilon), \tau_{\min }(n, \varepsilon)\right)
$$

for $n \geq 1$ we have

$$
\begin{aligned}
& E_{\vartheta}\left\|\zeta_{n, \varepsilon}\right\|^{q}=E_{\vartheta}\left(\sum_{i=0}^{p}\left\langle\zeta_{n, \varepsilon}\right\rangle_{i}^{2}\right)^{q / 2} \leq(p+1)^{\frac{q-2}{2}} b_{q} E_{\vartheta} \sum_{i=0}^{p}\left(\int_{\nu_{n}(\varepsilon)}^{\tau_{\min }(n, \varepsilon)} \tilde{b}_{i n}^{2}(t) d t\right)^{q / 2} \leq \\
& \leq(p+1)^{\frac{q-2}{2}} b_{q} E_{\vartheta} \sum_{j=0}^{m} \sum_{i=s_{j-1}+1}^{s_{j}}\left(\int_{\nu_{n}(\varepsilon)}^{\tau_{i}(n, \varepsilon)} \tilde{b}_{i n}^{2}(t) d t\right)^{q / 2} \leq(p+1)^{\frac{q-2}{2}}(m+1) b_{q}
\end{aligned}
$$

Thus we have got the wanted control of the moments of the noises mentioned in the Introduction. Note that for $q=2$ and $m=0$ we have the equalities

$$
E_{\vartheta}\left\|\tilde{\zeta}_{n, \varepsilon}\right\|^{2}=E_{\vartheta} \int_{\nu_{n}(\varepsilon)}^{\tau_{0}(n, \varepsilon)}\left\|\tilde{b}_{n}(t)\right\|^{2} d t=1, n \geq 1 .
$$

Put

$$
\bar{\tau}(n, \varepsilon)=(\underbrace{\tau_{0}(n, \varepsilon), \ldots, \tau_{0}(n, \varepsilon)}_{l_{0}}, \underbrace{\tau_{1}(n, \varepsilon), \ldots, \tau_{1}(n, \varepsilon)}_{l_{1}}, \ldots, \underbrace{\tau_{m}(n, \varepsilon), \ldots, \tau_{m}(n, \varepsilon)}_{l_{m}})
$$

and

$$
\tau_{\max }(n, \varepsilon)=\max \left\{\tau_{0}(n, \varepsilon), \tau_{1}(n, \varepsilon), \ldots, \tau_{m}(n, \varepsilon)\right\} .
$$

We shall prove below, that the vector-sequence $(\bar{\tau}(n, \varepsilon))$ belongs to the set $\Upsilon$.

The inequalities (18) suggest that the estimation of the parameter $\vartheta$ should be performed on the intervals $\left[\nu_{n}(\varepsilon), \tau_{\min }(n, \varepsilon)\right]$ with the weights $V(t)$ :

$$
\vartheta(n, \varepsilon)=\hat{\vartheta}\left(\nu_{n}(\varepsilon), \tau_{\min }(n, \varepsilon)\right), n \geq 1 .
$$

For the construction of sequential plan we put

$$
\sigma(\varepsilon)=\inf \{N \geq 1: S(N, \varepsilon) \geq \varrho\}
$$

where

$$
S(N, \varepsilon)=\sum_{n=1}^{N} \beta^{q}(n, \varepsilon)
$$

and $\beta(n, \varepsilon)$ is defined as

$$
\beta(n, \varepsilon)=\left\|G_{n, \varepsilon}^{-1}\right\|^{-1}
$$

if the matrix

$$
G_{n, \varepsilon}=\left(\varepsilon^{-1} c_{n}\right)^{-\frac{1}{2}} \tilde{\Psi}^{-\frac{1}{2}}(n, \varepsilon) G\left(\nu_{n}(\varepsilon), \tau_{\min }(n, \varepsilon)\right)
$$


is invertible; 0 in the other case,

$$
\varrho=b_{q}(p+1)^{\frac{q-2}{q}}(m+1) \sum_{n \geq 1} c_{n}^{-q / 2} .
$$

DEFINITION (D2) The sequential plan $(T(\varepsilon), \vartheta(\varepsilon))$ of estimation of the vector $\vartheta \in \Theta$ will be defined by the formulae

$$
T(\varepsilon)=\tau_{\max }(\sigma(\varepsilon), \varepsilon), \quad \vartheta(\varepsilon)=S^{-1}(\sigma(\varepsilon), \varepsilon) \sum_{n=1}^{\sigma(\varepsilon)} \beta^{q}(n, \varepsilon) \vartheta(n, \varepsilon),
$$

where $T(\varepsilon)$ is the duration of estimation, and $\vartheta(\varepsilon)$ is the estimator of $\vartheta$ with given accuracy $\varepsilon>0$.

By construction the sequential estimator $\vartheta(\varepsilon)$ is a random weighted mean of the weighted LSE's $\hat{\vartheta}(\cdot, \cdot)$, calculated on the intervals $\left[\nu_{n}(\varepsilon), \tau_{\min }(n, \varepsilon)\right], n \geq 1$.

The following theorem summarizes the main result concerning the sequential plan $(T(\varepsilon), \vartheta(\varepsilon))$

THEOREM 1. Suppose Assumptions $(V),(G),(\varphi \Psi),(\Psi)$ and $\left(\alpha_{1}\right)$ hold and the conditions (14)-(16) are fulfilled. Then for every $\varepsilon>0$ and every $\vartheta \in \Theta$ the sequential plan $(T(\varepsilon), \vartheta(\varepsilon))$ from Definition (D2) is closed, i.e. it holds $T(\varepsilon)<\infty P_{\vartheta}-$ a.s.

Moreover, the following statements are true:

$1^{\circ}$. for any $\varepsilon>0$ it holds

$$
\sup _{\vartheta \in \Theta}\|\vartheta(\varepsilon)-\vartheta\|_{q}^{2} \leq \varepsilon
$$

$2^{\circ}$. if, in addition, the Assumption $\left(\alpha_{2}\right)$ is valid, then for every $\vartheta \in \Theta$

$$
\text { a) } \varlimsup_{\varepsilon \rightarrow 0} h(\varepsilon) \cdot \varphi_{0}(T(\varepsilon))<\infty \quad P_{\vartheta}-\text { a.s. },
$$

where the function $h(\cdot)$ is defined in (33) below, and, moreover, if the condition (12) is valid, then

$$
\text { b) } \lim _{\varepsilon \rightarrow 0} \varepsilon \cdot \varphi_{0}(T(\varepsilon))>0 \quad P_{\vartheta}-\text { a.s.; }
$$

$3^{\circ}$. if $g(T)=o(T)$ as $T \rightarrow \infty$ then under the conditions from $\left.2^{\circ} b\right)$ the estimator $\vartheta(\varepsilon)$ is strongly consistent:

$$
\lim _{\varepsilon \rightarrow 0} \vartheta(\varepsilon)=\vartheta \quad P_{\vartheta}-\text { a.s. }
$$

Proof. Fix an arbitrary $\vartheta \in \Theta$. Let us verify the finiteness of $T(\varepsilon)=\tau_{\max }(\sigma(\varepsilon), \varepsilon)$. While the stopping times $\tau_{i}(n, \varepsilon)$ due to Assumption (V) are finite for all $i=$ $\overline{0, m}, n \geq 1$ and $\varepsilon>0$, it suffices to establish the finiteness of the stopping times $\sigma(\varepsilon)$.

From Assumptions $(\mathrm{V}),(\varphi \psi),(\alpha 1),(114),(14)$ and the representation

$$
\sum_{i=s_{j-1}+1}^{s_{j}}\left(\frac{\Psi_{i}\left(\alpha, \varphi_{0}\left(\tau_{j}(n, \varepsilon)\right)\right)}{\Psi_{i i}(\alpha, n, \varepsilon)}\right)^{q / 2}\left(\frac{\Psi_{i i}(\alpha, n, \varepsilon)}{\tilde{\Psi}_{i i}(n, \varepsilon)}\right)^{q / 2} .
$$




$$
\cdot\left(\frac{1}{\varphi_{i}\left(\tau_{j}(n, \varepsilon)\right)} \int_{\nu_{n}(\varepsilon)}^{\tau_{j}(n, \varepsilon)} b_{i}^{2}(t) d t\right)^{q / 2}=1
$$

$j=\overline{0, m}$ which is only the form of the definition (17), it follows that $P_{\vartheta}-$ a.s.

$$
\sum_{i=s_{j-1}+1}^{s_{j}}\left(\frac{\Psi_{i}\left(\alpha, \varphi_{0}\left(\tau_{j}(n, \varepsilon)\right)\right)}{\Psi_{i}\left(\alpha, \varepsilon^{-1} c_{n}\right)}\right)^{q / 2} \simeq C \text { as } n \rightarrow \infty,
$$

$j=\overline{0, m}$ and, as follows,

$$
\sum_{i=s_{j-1}+1}^{s_{j}} \frac{\Psi_{i}\left(\alpha, \varphi_{0}\left(\tau_{j}(n, \varepsilon)\right)\right)}{\Psi_{i}\left(\alpha, \varepsilon^{-1} c_{n}\right)} \simeq C \text { as } n \rightarrow \infty,
$$

$j=\overline{0, m}$. Then, by the definition, the functions $\varphi_{i}\left(\tau_{j}(n, \varepsilon)\right) \in Y\left(\Psi\left[\alpha, \bar{i}_{l_{j}}\right]\right), \bar{i}_{l_{j}}=$ $\left(s_{j-1}+1, \ldots, s_{j}\right), i=\overline{s_{j-1}+1, s_{j}}, j=\overline{0, m}$ and, according to the definition of $\bar{i}_{l_{j}}$ and the property $\Psi\left[\alpha, \bar{i}_{l_{j}}\right] \in P_{l_{j}}(\alpha)$, the functions $\varphi_{i}\left(\tau_{j}(n, \varepsilon)\right) \in Y^{\prime}\left(\Psi\left[\alpha, \bar{i}_{l_{j}}\right]\right)$ and the following relations for $i=\overline{s_{j-1}+1, s_{j}}, j=\overline{0, m} P_{\vartheta}$ - a.s. hold true:

$$
\frac{\Psi_{i}\left(\alpha, \varphi_{0}\left(\tau_{j}(n, \varepsilon)\right)\right)}{\Psi_{i}\left(\alpha, \varepsilon^{-1} c_{n}\right)} \simeq C \text { as } n \rightarrow \infty
$$

Then, take into account Assumption $(\Psi)$, from (21) we obtain with $P_{\vartheta}$ - probability one:

$$
\frac{\varphi_{0}\left(\tau_{j}(n, \varepsilon)\right)}{\varepsilon^{-1} c_{n}} \simeq C \text { as } n \rightarrow \infty, \quad j=\overline{0, m} .
$$

Then for $m>0$ we have

$$
\begin{aligned}
& \frac{\varphi_{0}\left(\tau_{\min }(n, \varepsilon)\right)}{\varepsilon^{-1} c_{n}} \simeq C \text { as } n \rightarrow \infty P_{\vartheta}-\text { a.s. } \\
& \frac{\varphi_{0}\left(\tau_{i}(n, \varepsilon)\right)}{\varphi_{0}\left(\tau_{j}(n, \varepsilon)\right)} \simeq C \text { as } n \rightarrow \infty, \quad P_{\vartheta}-\text { a.s. }
\end{aligned}
$$

$i, j=\overline{0, m}$ and, as follows,

$$
\frac{\varphi_{0}\left(\tau_{\max }(n, \varepsilon)\right)}{\varphi_{0}\left(\tau_{\min }(n, \varepsilon)\right)} \simeq C \text { as } n \rightarrow \infty P_{\vartheta}-\text { a.s. }
$$

Then, by the definition (13), the vector-sequence $(\bar{\tau}(n, \varepsilon))$ belongs to the set $\Upsilon$.

From (21), (24) and Assumption $(\alpha 1)$ we can get with $P_{\vartheta}$-probability one, for all $i=\overline{0, p}$, the relations

$$
\left\langle\bar{\varphi}^{-1}\left(\tau_{\min }(n, \varepsilon)\right) \tilde{\Psi}(n, \varepsilon)\right\rangle_{i i} \simeq C \text { as } n \rightarrow \infty .
$$

For sufficiently simple and smooth functions $\Psi_{i}$ (see, for example, Tables 3 and 6 below), the relations (26) lead to knowledge of exact asymptotic behavior for the 
stopping times $\tau_{i}(n, \varepsilon), i=\overline{0, m}$, see examples below.

From (14) and (23) it follows, that $\varphi_{0}\left(\nu_{n}(\varepsilon)\right)=o\left(\bar{g}^{-1 / 2}\left(\tau_{\min }(n, \varepsilon)\right) \varphi_{0}\left(\tau_{\min }(n, \varepsilon)\right)\right)$ as $n \rightarrow \infty \quad P_{\vartheta}-$ a.s. and, by the definition of the class $\overline{\mathcal{G}}_{1}$, we obtain:

$$
\nu_{n}(\varepsilon)=o\left(\tau_{\min }(n, \varepsilon)\right) \text { as } n \rightarrow \infty P_{\vartheta}-\text { a.s. }
$$

Note, that all the obtained relations $(21)-(27)$ are also true $P_{\vartheta}$-a.s. under Assumption $(\alpha 2)$ for every $n \geq 1$ as $\varepsilon \rightarrow 0$. From (25) it follows, that the vectorsequence $(\bar{\tau}(n, \varepsilon))$ belongs to the set $\Upsilon$ as a function of $n$ or $\varepsilon P_{\vartheta}$-a.s.

In the sequel, we denote $c_{i}, C_{i}, c_{i j}, C_{i j}, \tilde{C}_{i}, \tilde{C}_{i j}, \bar{C}_{i j}, \ldots i, j,=1,2, \ldots$ nonnegative constants or random numbers, and $c_{i j}(T), C_{i j}(T), \tilde{C}_{i j}(T), \bar{C}_{i j}(T), \ldots i, j,=1$, $2, \ldots$ nonnegative continuous periodic functions, possibly random and different even within the same index.

By making use of the relations (10), (23), (26) and (27) and definition (19) of functions $\beta(n, \varepsilon)$ we get the lower limiting bound for $n$ large enough for these functions:

$$
\begin{gathered}
\beta^{2}(n, \varepsilon)=\left\|G_{n, \varepsilon}^{-1}\right\|^{-2}=\left(\varepsilon^{-1} c_{n}\right)^{-1} \cdot\left\|G^{-1}\left(\nu_{n}(\varepsilon), \tau_{\min }(n, \varepsilon)\right) \tilde{\Psi} \frac{1}{2}(n, \varepsilon)\right\|^{-2}=\left(\varepsilon^{-1} c_{n}\right)^{-1} \cdot \\
\cdot \varphi_{0}\left(\tau_{\min }(n, \varepsilon)\right) \cdot\left\|\tilde{G}^{-1}\left(\nu_{n}(\varepsilon), \tau_{\min }(n, \varepsilon)\right) \cdot\left(\bar{\varphi}^{-1}\left(\tau_{\min }(n, \varepsilon)\right) \tilde{\Psi}(n, \varepsilon)\right)^{\frac{1}{2}}\right\|^{-2} \geq \\
\geq C_{1} \cdot\left\|\tilde{G}^{-1}\left(\nu_{n}(\varepsilon), \tau_{\min }(n, \varepsilon)\right)\right\|^{-2} \cdot\left\|\bar{\varphi}^{-1}\left(\tau_{\min }(n, \varepsilon)\right) \tilde{\Psi}(n, \varepsilon)\right\|^{-1} \geq \\
\geq C_{2} \cdot\left\|\tilde{G}^{-1}\left(\nu_{n}(\varepsilon), \tau_{\min }(n, \varepsilon)\right)\right\|^{-2} \geq \frac{C}{\bar{g}\left(\tau_{\min }(n, \varepsilon)\right)} \geq \frac{\beta_{\star}}{g\left(\varepsilon^{-1} c_{n}\right)} \quad P_{\vartheta}-\text { a.s., } \quad \text { (28) }
\end{gathered}
$$

where $\beta_{\star}$ is some $P_{\vartheta}-$ a.s. positive and finite random number.

Then for all $\varepsilon>0$, according to (16), the stopping times $\sigma(\varepsilon)$ and $T(\varepsilon)$ are finite $P_{\vartheta}-$ a.s.

Analogously, using the condition (12) and relations (11), (23), (26), (27), for some $P_{\vartheta}-$ a.s. positive and finite random number $\beta^{*}$ we can obtain the inequalities with $P_{\vartheta}$-probability one

$$
\begin{gathered}
\beta^{2}(n, \varepsilon)=\left(\varepsilon^{-1} c_{n}\right)^{-1} \varphi_{0}\left(\tau_{\min }(n, \varepsilon)\right) \cdot \\
\cdot\left\|\tilde{G}^{-1}\left(\nu_{n}(\varepsilon), \tau_{\min }(n, \varepsilon)\right) \cdot\left(\bar{\varphi}^{-1}\left(\tau_{\min }(n, \varepsilon)\right) \tilde{\Psi}(n, \varepsilon)\right)^{\frac{1}{2}}\right\|^{-2}= \\
=\left(\varepsilon^{-1} c_{n}\right)^{-1} \varphi_{0}\left(\tau_{\min }(n, \varepsilon)\right) \cdot\left\{\operatorname { t r } \left[\tilde{G}^{-1}\left(\nu_{n}(\varepsilon), \tau_{\min }(n, \varepsilon)\right) \cdot\right.\right. \\
\left.\left.\cdot \bar{\varphi}^{-1}\left(\tau_{\min }(n, \varepsilon)\right) \tilde{\Psi}(n, \varepsilon) \cdot\left(\tilde{G}^{\prime}\left(\nu_{n}(\varepsilon), \tau_{\min }(n, \varepsilon)\right)\right)^{-1}\right]\right\}^{-1} \leq \\
\leq\left(\varepsilon^{-1} c_{n}\right)^{-1} \varphi_{0}\left(\tau_{\min }(n, \varepsilon)\right) \cdot \lambda_{\min }^{-1}\left\{\bar{\varphi}^{-1}\left(\tau_{\min }(n, \varepsilon)\right) \tilde{\Psi}(n, \varepsilon)\right\} \cdot \\
\cdot \lambda_{\max }^{-1}\left\{\tilde{G}^{-1}\left(\nu_{n}(\varepsilon), \tau_{\min }(n, \varepsilon)\right) \cdot\left(\tilde{G}^{\prime}\left(\nu_{n}(\varepsilon), \tau_{\min }(n, \varepsilon)\right)\right)^{-1}\right\} \leq \\
\leq(p+1)\left(\varepsilon^{-1} c_{n}\right)^{-1} \varphi_{0}\left(\tau_{\min }(n, \varepsilon)\right) \cdot \lambda_{\max }\left(\bar{\varphi}\left(\tau_{\min }(n, \varepsilon)\right) \cdot\right. \\
\left.\cdot \tilde{\Psi}^{-1}(n, \varepsilon)\right)\left\|\tilde{G}^{-1}\left(\nu_{n}(\varepsilon), \tau_{\min }(n, \varepsilon)\right)\right\|^{-2} \leq \beta^{*}<\infty
\end{gathered}
$$

for $n$ large enough.

In a similar way, using the Assumption $(\alpha 2)$, we can get the inequalities (28) for every $n \geq 1$ and small enough $\varepsilon$ and, using in addition the condition (12), the 
inequalities (29).

$1^{\circ}$. Now we estimate the $L_{q}$-norm of the deviation of $\vartheta(\varepsilon)$. From (1) and the definition (20) it follows that

$$
\|\vartheta(\varepsilon)-\vartheta\|_{q}^{2}=\left(E_{\vartheta}\left(S^{-1}(\sigma(\varepsilon), \varepsilon) \cdot\left\|\sum_{n=1}^{\sigma(\varepsilon)} \beta^{q}(n, \varepsilon)(\vartheta(n, \varepsilon)-\vartheta)\right\|\right)^{q}\right)^{\frac{2}{q}} .
$$

According to the Hölder inequality

$$
\sum_{n} a_{n} b_{n} \leq\left(\sum_{n} a_{n}^{\frac{q}{q-1}}\right)^{\frac{q-1}{q}}\left(\sum_{n} b_{n}^{q}\right)^{\frac{1}{q}}
$$

where we put $a_{n}=\beta^{q-1}(n, \varepsilon)$ and $b_{n}=\beta(n, \varepsilon)\|\vartheta(n)-\vartheta\|$, we may enlarge this expression and continue the estimations by

$$
\begin{gathered}
\|\vartheta(\varepsilon)-\vartheta\|_{q}^{2} \leq\left(E_{\vartheta} S^{-q}(\sigma(\varepsilon), \varepsilon) \cdot\left(\sum_{n \geq 1} \beta^{q}(n, \varepsilon)\|\vartheta(n, \varepsilon)-\vartheta\|\right)^{q}\right)^{\frac{2}{q}} \leq \\
\leq\left(E_{\vartheta} S^{-1}(\sigma(\varepsilon), \varepsilon) \sum_{n \geq 1} \beta^{q}(n, \varepsilon)\|\vartheta(n, \varepsilon)-\vartheta\|^{q}\right)^{\frac{2}{q}} .
\end{gathered}
$$

Then by definitions of $\sigma(\varepsilon), \beta(n, \varepsilon), \tilde{\Psi}(n, \varepsilon), \varrho$ and from (18) we have

$$
\begin{gathered}
\|\vartheta(\varepsilon)-\vartheta\|_{q}^{2} \leq\left(\varrho^{-1} \sum_{n \geq 1} E_{\vartheta} \beta^{q}(n, \varepsilon) \cdot\left\|G^{-1}\left(\nu_{n}(\varepsilon), \tau_{\min }(n, \varepsilon)\right) \cdot \zeta\left(\nu_{n}(\varepsilon), \tau_{\min }(n, \varepsilon)\right)\right\|^{q}\right)^{\frac{2}{q}}= \\
=\left(\varrho^{-1} \sum_{n \geq 1} E_{\vartheta} \beta^{q}(n, \varepsilon)\left(\varepsilon^{-1} c_{n}\right)^{-\frac{q}{2}}\left\|G_{n, \varepsilon}^{-1} \cdot \zeta_{n, \varepsilon}\right\|^{q}\right)^{\frac{2}{q}} \leq \\
\leq \varepsilon\left(\varrho^{-1} \sum_{n \geq 1} c_{n}^{-\frac{q}{2}} E_{\vartheta} \beta^{q}(n, \varepsilon)\left\|G_{n, \varepsilon}^{-1}\right\|^{q} \cdot\left\|\zeta_{n, \varepsilon}\right\|^{q}\right)^{\frac{2}{q}}= \\
=\varepsilon\left(\varrho^{-1} \sum_{n \geq 1} c_{n}^{-q / 2} E_{\vartheta}\left\|\zeta_{n, \varepsilon}\right\|^{q}\right)^{\frac{2}{q}} \leq \varepsilon\left(\varrho^{-1}(p+1)^{\frac{q-2}{q}} \cdot(m+1) b_{q} \sum_{n \geq 1} c_{n}^{-q / 2}\right)^{\frac{2}{q}}=\varepsilon .
\end{gathered}
$$

$2^{\circ}$. The second assertion follows from the Definition (D2) of $T(\varepsilon)$, the definition (19) of $\sigma(\varepsilon)$, Assumptions (G) and $(\alpha 2)$, condition (12) and relations (22), (28), (29). Indeed, according to (22) for $\varepsilon \rightarrow 0$ under the Assumption $(\alpha 2)$ for every $n \geq 1$

$$
\varepsilon \varphi_{0}\left(\tau_{\max }(n, \varepsilon)\right) \simeq C \text { as } \varepsilon \rightarrow 0 \quad P_{\vartheta}-\text { a.s. }
$$

Denote

$$
\begin{gathered}
\sigma_{1}=\inf \left\{N \geq 1: N \geq \varrho\left(\beta^{\star}\right)^{-q / 2}\right\}, \\
\sigma_{2}(\varepsilon)=\inf \left\{N \geq 1: \sum_{i=1}^{N} g^{-q / 2}\left(\varepsilon^{-1} c_{n}\right)>\varrho \beta_{\star}^{-q / 2}\right\} .
\end{gathered}
$$

Using the definition (19) of $\sigma(\varepsilon)$ and (28) for $\varepsilon$ small enough we have

$$
\sigma(\varepsilon) \leq \sigma_{2}(\varepsilon) \quad P_{\vartheta}-\text { a.s. }
$$


and, in addition, under the condition (12) from (29) for $\varepsilon$ small enough we obtain

$$
\sigma(\varepsilon) \geq \sigma_{1} \quad P_{\vartheta}-\text { a.s. }
$$

Denote

$$
h(\varepsilon)=\varepsilon c_{\sigma_{2}(\varepsilon)}^{-1} .
$$

Take into account, that by Definition (D2), $T(\varepsilon)=\tau_{\max }(\sigma(\varepsilon), \varepsilon)$ and from the relations (30)-(32) we obtain the second assertion of Theorem 1:

$$
\varlimsup_{\varepsilon \rightarrow 0} h(\varepsilon) \cdot \varphi_{0}(T(\varepsilon))<\infty \quad \text { as } \varepsilon \rightarrow 0 \quad P_{\vartheta}-\text { a.s. }
$$

and

$$
\lim _{\varepsilon \rightarrow 0} \varepsilon \cdot \varphi_{0}(T(\varepsilon))>0 \quad \text { as } \varepsilon \rightarrow 0 \quad P_{\vartheta}-\text { a.s. }
$$

The lower and upper bounds exist under the Assumption (G) and, in addition, under the condition (12) respectively.

Note, that for the constant function $g(\cdot) \equiv$ const, the stopping time $\sigma_{2}(\varepsilon) \simeq$ $C$ as $\varepsilon \rightarrow 0 \quad P_{\vartheta}-$ a.s. and in this case we have

$$
\varepsilon \cdot \varphi_{0}(T(\varepsilon)) \simeq C \text { as } \varepsilon \rightarrow 0 \quad P_{\vartheta}-\text { a.s. }
$$

$3^{\circ}$. First we establish the strong consistency of $\vartheta(n, \varepsilon)$ as $\varepsilon \rightarrow 0$. By the definition of $\vartheta(n, \varepsilon)$ we can write

$$
\begin{gathered}
\vartheta(n, \varepsilon)-\vartheta=\left(\varepsilon^{-1} c_{n}\right)^{-1 / 2} G_{n, \varepsilon}^{-1} \zeta_{n, \varepsilon}=\left[\varphi_{0}\left(\tau_{\min }(n, \varepsilon)\right) \cdot \varepsilon c_{n}^{-1}\right]^{1 / 2} \cdot\left[\bar{g}^{-1 / 2}\left(\tau_{\min }(n, \varepsilon)\right) \cdot G_{n, \varepsilon}^{-1}\right] \cdot \\
\cdot\left[\tilde{\Psi}^{-1}(n, \varepsilon) \bar{\varphi}\left(\tau_{\min }(n, \varepsilon)\right)\right]^{1 / 2} \cdot\left[\bar{g}^{1 / 2}\left(\tau_{\min }(n, \varepsilon)\right) \cdot\right. \\
\left.\cdot \varphi_{0}^{-1 / 2}\left(\tau_{\min }(n, \varepsilon)\right) \bar{\varphi}^{-1 / 2}\left(\tau_{\min }(n, \varepsilon)\right) \cdot \zeta\left(\nu_{n}(\varepsilon), \tau_{\min }(n, \varepsilon)\right)\right] .
\end{gathered}
$$

According to (23), (26) and (28) the first three factors in the right-hand side of this equality are bounded $P_{\vartheta}-$ a.s. on the norm from above for $\varepsilon$ small enough or $n$ large enough.

In the sequel we will use the notation $\lim _{n \vee \varepsilon}$ which means, that the corresponding relation holds for $\lim _{n \rightarrow \infty}$ as well as for $\lim _{\varepsilon \rightarrow 0}$.

The last factor vanishes in $P_{\vartheta}-$ a.s. sense in view of $(27)$, condition $g(T)=$ $o(T), T \rightarrow \infty$ of Theorem 1, and by the properties of the square integrable martingales $\zeta_{i}(0, T)$ for all $i=\overline{0, p}$ :

$\lim _{n \vee \varepsilon} \frac{\bar{g}^{1 / 2}\left(\tau_{\min }(n, \varepsilon)\right) \cdot \zeta_{i}\left(\nu_{n}(\varepsilon), \tau_{\min }(n, \varepsilon)\right)}{\varphi_{0}^{1 / 2}\left(\tau_{\min }(n, \varepsilon)\right) \cdot \bar{\varphi}_{i i}^{1 / 2}\left(\tau_{\min }(n, \varepsilon)\right)}=\lim _{T \rightarrow \infty} \frac{g^{1 / 2}\left(\varphi_{0}(T)\right) \cdot \zeta_{i}(0, T)}{\varphi_{0}^{1 / 2}(T) \cdot \bar{\varphi}_{i i}^{1 / 2}(T)}=0 P_{\vartheta}$-a.s.

Then the estimators $\vartheta(n, \varepsilon)$ are strongly consistent as $\varepsilon \rightarrow 0$ for every $n \geq 1$ and as $n \rightarrow \infty$ for every $\varepsilon>0$.

Moreover, taking into account for $\varepsilon$ small enough the relations (29)-(32) for the weights $\beta(n, \varepsilon)$ and times $\sigma(\varepsilon)$ we can see that the weighted arithmetical mean $\vartheta(\varepsilon)$ of estimators $\vartheta(n, \varepsilon)$ is strongly consistent as well.

Hence Theorem 1 is proved. 


\section{Sequential parameter estimation of an autoregressive process}

As an application, in this section we will use the general estimation procedure, presented in Section 2 for sequential parameter estimation of a second-order autoregressive process.

Define $p=1, x(t)=\dot{x}_{t}, a_{0}(t)=\dot{x}_{t}, a_{1}(t)=x_{t}$. Then the equation (1) has the form (3):

$$
d \dot{x}_{t}=\vartheta_{0} \dot{x}_{t} d t+\vartheta_{1} x_{t} d t+d W(t), t \geq 0 .
$$

Denote by $\lambda_{0}$ and $\lambda_{1}$ the roots of its characteristic polynomial

$$
q(\lambda)=\lambda^{2}-\vartheta_{0} \lambda-\vartheta_{1} .
$$

Now we write equation (34) in the matrix form:

$$
d X(t)=A X(t) d t+B d W(t)
$$

where

$$
A=\left(\begin{array}{ll}
\vartheta_{0} & \vartheta_{1} \\
1 & 0
\end{array}\right), \quad X(t)=\left(\begin{array}{c}
\dot{x}_{t} \\
x_{t}
\end{array}\right), \quad B=\left(\begin{array}{l}
1 \\
0
\end{array}\right) .
$$

It is obviously, that the roots $\lambda_{0}, \lambda_{1}$ are the eigenvalues of the matrix $A$ and

$$
\lambda_{0}=\frac{\vartheta_{0}}{2}+\sqrt{\left(\frac{\vartheta_{0}}{2}\right)^{2}+\vartheta_{1}}, \quad \lambda_{1}=\frac{\vartheta_{0}}{2}-\sqrt{\left(\frac{\vartheta_{0}}{2}\right)^{2}+\vartheta_{1}} .
$$

For this model we can define the following parametric sets

$$
\begin{gathered}
\tilde{\Theta}_{1}=\tilde{\Theta}_{11} \cup \tilde{\Theta}_{12} \cup \tilde{\Theta}_{13} \cup \tilde{\Theta}_{14}, \\
\tilde{\Theta}_{11}=\left\{\vartheta \in R^{2}: \vartheta_{0}<0, \vartheta_{1}<0\right\}, \\
\tilde{\Theta}_{12}=\left\{\vartheta \in R^{2}: \vartheta_{0}>0, \vartheta_{1}<-\left(\vartheta_{0} / 2\right)^{2}\right\}, \\
\tilde{\Theta}_{13}=\left\{\vartheta \in R^{2}: \vartheta_{0}=0, \vartheta_{1}<0\right\}, \\
\tilde{\Theta}_{14}=\left\{\vartheta \in R^{2}: \vartheta_{0}>0, \vartheta_{1}=-\left(\vartheta_{0} / 2\right)^{2}\right\}, \\
\tilde{\Theta}_{2}=\left\{\vartheta \in R^{2}: \vartheta_{0}>0,-\left(\vartheta_{0} / 2\right)^{2}<\vartheta_{1}<0\right\}, \\
\tilde{\Theta}_{3}=\left\{\vartheta \in R^{2}: \vartheta_{1}>0\right\}
\end{gathered}
$$

and we put

$$
\tilde{\Theta}=\tilde{\Theta}_{1} \cup \tilde{\Theta}_{2} \cup \tilde{\Theta}_{3}=R^{2} \backslash\left\{\vartheta \in R^{2}: \vartheta_{1}=0\right\} .
$$

Remark 3.1. As usual, the condition $\vartheta_{1} \neq 0$ means the knowledge of the order $(p=1)$ of the process (34). It should be noted that the problem of sequential estimation for the case $\tilde{\Theta} \backslash\left\{\vartheta \in R^{2}: \vartheta_{0}=0\right\}$ has been solved, in principle, in [6], [7].

Now we show, that the smallest $\lambda_{\min }(G(T))$ and the largest $\lambda_{\max }(G(T))$ eigenvalues respectively of the empirical information matrix $G(T)=\int_{0}^{T} X(t) X^{\prime}(t) d t$ have the given in Table 1 asymptotic rates of increase: 
Table 1

\begin{tabular}{|l||l|l|}
\hline Region & $\lambda_{\min }(G(T))$ & $\lambda_{\max }(G(T))$ \\
\hline$\tilde{\Theta}_{11}$ & $T$ & $T$ \\
\hline$\tilde{\Theta}_{12}$ & $\mathrm{e}^{\vartheta_{0} T}$ & $\mathrm{e}^{\vartheta_{0} T}$ \\
\hline$\tilde{\Theta}_{13}$ & $T^{2}$ & $T^{2}$ \\
\hline$\tilde{\Theta}_{14}$ & $T^{-2} \mathrm{e}^{\vartheta_{0} T}$ & $T^{2} \mathrm{e}^{\vartheta_{0} T}$ \\
\hline$\tilde{\Theta}_{2}$ & $\mathrm{e}^{2 \lambda_{1} T}$ & $\mathrm{e}^{2 \lambda_{0} T}$ \\
\hline$\tilde{\Theta}_{3}$ & $T$ & $\mathrm{e}^{\vartheta_{0} T}$ \\
\hline
\end{tabular}

We shall find the weight matrices $V$ and $V(t)$ for all the defined regions. Introduce following notation: $v_{i}=\operatorname{Re} \lambda_{i}, \beta=\operatorname{Im} \lambda_{i}, i=0,1$.

The sets $\tilde{\Theta}_{11}, \ldots, \tilde{\Theta}_{14}, \tilde{\Theta}_{2}, \tilde{\Theta}_{3}$, defined above can be rewritten in terms of eigenvalues $\lambda_{0}, \lambda_{1}$ in the following form:

$\tilde{\Theta}_{11}=\left\{\vartheta \in R^{2}:-\infty<v_{1} \leq v_{0}<0\right\}, \quad \tilde{\Theta}_{12}=\left\{\vartheta \in R^{2}: 0<v_{1}=v_{0}<\infty, \beta \neq 0\right\}$,

$\tilde{\Theta}_{13}=\left\{\vartheta \in R^{2}: v_{0}=0, \beta \neq 0\right\}, \quad \tilde{\Theta}_{14}=\left\{\vartheta \in R^{2}: 0<v_{1}=v_{0}<\infty, \beta=0\right\}$,

$\tilde{\Theta}_{2}=\left\{\vartheta \in R^{2}: 0<v_{1}<v_{0}<\infty\right\}, \quad \tilde{\Theta}_{3}=\left\{\vartheta \in R^{2}:-\infty<v_{1}<0<v_{0}<\infty\right\}$.

The linear rates of increase for $\lambda_{\min }(G(T))$ and $\lambda_{\max }(G(T))$ in the case $\tilde{\Theta}_{11}$ follow from the equality

$$
\lim _{T \rightarrow \infty} T^{-1} G(T)=F_{11} \quad P_{\vartheta}-a . s .
$$

obtained in [7], where $F_{11}$ is a non-degenerate constant matrix. Assumption (V) is fulfilled if we put $\varphi_{0}(T)=\varphi_{1}(T)=T, V(t) \equiv V=I$.

From Lemma 3 in [7] it follows, that for $\vartheta \in \tilde{\Theta}_{12} \cup \tilde{\Theta}_{14}$

$$
\lim _{T \rightarrow \infty} \mathrm{e}^{-A T} G(T) \mathrm{e}^{-A^{\prime} T}=F_{12} \quad P_{\vartheta}-\text { a.s. }
$$

where

$$
F_{12}=\int_{0}^{\infty} \mathrm{e}^{-A t} U U^{\prime} \mathrm{e}^{-A^{\prime} t} d t, \quad U=\int_{0}^{\infty} \mathrm{e}^{-A t} d W(t) B
$$

and $F_{12}$ is random $P_{\vartheta}-$ a.s. positive definite matrix.

Consider the case $\tilde{\Theta}_{12}$ of complex eigenvalues $\lambda_{0}=v_{0}+i \beta, \lambda_{1}=v_{0}-i \beta, v_{0}=$ $\frac{\vartheta_{0}}{2}, \beta=\sqrt{\left(\frac{\vartheta_{0}}{2}\right)^{2}+\vartheta_{1}}$. In this case we can find the diagonal form of the matrix $A$

$$
A=\tilde{Q}_{12}^{-1}\left(\begin{array}{ll}
\lambda_{1} & 0 \\
0 & \lambda_{0}
\end{array}\right) \tilde{Q}_{12}, \text { where } \tilde{Q}_{12}=\left(\begin{array}{cc}
\lambda_{1} & \vartheta_{1} \\
\lambda_{0} & \vartheta_{1}
\end{array}\right) .
$$

Then in the case $\tilde{\Theta}_{12}$, using of the equality (37) and the representation (38), we have with $P_{\vartheta}$-probability one

$$
\lim _{T \rightarrow \infty}\left\|\mathrm{e}^{-2 v_{0} T} G(T)-F_{12}(T)\right\|=0,
$$


where, for $T \geq 0$,

$$
\begin{gathered}
F_{12}(T)=\tilde{Q}_{12} J_{12}^{-1}(T) \tilde{Q}_{12}^{-1} F_{12}\left(\tilde{Q}_{12}^{\prime}\right)^{-1} J_{12}^{-1}(T) \tilde{Q}_{12}^{\prime}, \\
J_{12}(T)=\left(\begin{array}{ll}
(\cos \beta T+i \sin \beta T) & 0 \\
0 & (\cos \beta T-i \sin \beta T)
\end{array}\right) .
\end{gathered}
$$

The eigenvalues of the limiting positive definite matrix function $F_{12}(T)$ have positive finite constant bounds. As follows, Assumption $(\mathrm{V})$ is fulfilled with $\varphi_{0}(T)=$ $\varphi_{1}(T)=\mathrm{e}^{2 v_{0} T}, V(t) \equiv V=I$.

Consider the case $\tilde{\Theta}_{13}$. The following diagonal representation for the matrix $A$

$$
A=Q_{13}\left(\begin{array}{ll}
i \beta & 0 \\
0 & -i \beta
\end{array}\right) Q_{13}^{-1}, \quad Q_{13}=\left(\begin{array}{ll}
i \beta & -i \beta \\
1 & 1
\end{array}\right), \quad \beta=\sqrt{\left|\vartheta_{1}\right|}
$$

holds true. Then $\mathrm{e}^{A t}=Q_{13} J_{12}(t) Q_{13}^{-1}$ and

$$
X(t)=Q_{13} J_{12}(t) Q_{13}^{-1} X(0)+Q_{13} J_{13}(t) Q_{13}^{-1} B
$$

where

$$
\begin{gathered}
J_{13}(t)=\left(\begin{array}{ll}
A_{t}^{\prime} m_{t} & 0 \\
0 & B_{t}^{\prime} m_{t}
\end{array}\right), \quad m_{t}=\left(\begin{array}{l}
\xi_{t} \\
\eta_{t}
\end{array}\right), \\
A_{t}=\left(\begin{array}{l}
\cos \beta t+i \sin \beta t \\
-\sin \beta t-i \cos \beta t
\end{array}\right), \quad \xi_{t}=\int_{0}^{t} \cos \beta s d W(s), \\
B_{t}=\left(\begin{array}{l}
\cos \beta t-i \sin \beta t \\
-\sin \beta t+i \cos \beta t
\end{array}\right), \eta_{t}=\int_{0}^{t} \sin \beta s d W(s) .
\end{gathered}
$$

Using the strong law for martingales (see [16]), we can obtain the following $P_{\vartheta}-$ a.s. equation:

$$
\lim _{t \rightarrow \infty}\left\|t^{-1} X(t) X^{\prime}(t)-R(t)\right\|=0
$$

where

$$
R(t)=\frac{1}{4 \beta^{2}} Q_{13}\left(\begin{array}{ll}
-r(t) & 1 \\
1 & -\bar{r}(t)
\end{array}\right) Q_{13}^{\prime}, \quad r(t)=\frac{1-\beta^{2}}{1+\beta^{2}} \cos 2 \beta t+i \sin 2 \beta t .
$$

From (40) it follows that $P_{\vartheta}-$ a.s.

$$
\lim _{T \rightarrow \infty} \frac{1}{T^{2}} G(T)=F_{13},
$$

where

$$
F_{13}=\frac{1}{8 \beta^{2}} Q_{13}\left(\begin{array}{cc}
0 & 1 \\
1 & 0
\end{array}\right) Q_{13}^{\prime}
$$

and Assumption (V) holds with $\varphi_{0}(T)=\varphi_{1}(T)=T^{2}, V(t) \equiv V=I$.

In the case $\tilde{\Theta}_{14}$ we can find the following Jordan form for the matrix $A$ :

$$
A=Q_{14}^{-1}\left(\begin{array}{ll}
v_{0} & 1 \\
0 & v_{0}
\end{array}\right) Q_{14}, \quad Q_{14}=\left(\begin{array}{cl}
1 & \left(1-v_{0}\right) \\
1 & -v_{0}
\end{array}\right)
$$


and, as follows, the representation for the matrix exponent

$$
\mathrm{e}^{-A T}=Q_{14}^{-1}\left(\begin{array}{cc}
1 & t \\
0 & 1
\end{array}\right) Q_{14} \mathrm{e}^{-v_{0} t} .
$$

Then, from (37), denoting

$$
S_{14}=\left(\begin{array}{cc}
-v_{0} & v_{0}^{2} \\
-1 & v_{0}
\end{array}\right)
$$

we obtain with $P_{\vartheta}$-probability one,

$$
\begin{gathered}
\lim _{T \rightarrow \infty} \frac{1}{T^{2} \mathrm{e}^{2 v_{0} T}} G(T)=F_{14}, F_{14}=S_{14} F_{12} S_{14}^{\prime}, \\
\lim _{T \rightarrow \infty} \frac{1}{\mathrm{e}^{4 v_{0} T}} \operatorname{det}(G(T))=v_{0}^{4} \operatorname{det}\left(F_{12}\right) .
\end{gathered}
$$

In the sequel we say that functions $f$ and $g$ are equivalent asymptotically for $T$ large enough $(f(T) \sim g(T))$ if $f(T) / g(T) \rightarrow C$ as $T \rightarrow \infty$ for some positive number $C$.

From (42) and (43) it follows that $\lambda_{\max }(G(T)) \sim T^{2} \mathrm{e}^{2 v_{0} T}, \lambda_{\min }(G(T)) \sim T^{-2} \mathrm{e}^{2 v_{0} T}$ in the case $\tilde{\Theta}_{14}$ and Assumption (V) holds true if we put $\varphi_{0}(T)=\varphi_{1}(T)=T^{2} \mathrm{e}^{2 v_{0} T}$, $V(t) \equiv V=I$.

Consider the cases $\tilde{\Theta}_{2}$ and $\tilde{\Theta}_{3}$. Denote

$$
Q_{23}=\left(\begin{array}{c}
Q_{0} \\
Q_{1}
\end{array}\right), \quad Q_{0}=\left(1,-v_{0}\right), \quad Q_{1}=\left(1,-v_{1}\right),
$$

$q_{0}(\lambda)=\lambda-v_{0}, q_{1}(\lambda)=\lambda-v_{1}$.

Following an idea of [14], in the case of real eigenvalues $\lambda_{i}=v_{i}, i=0,1$ the characteristic polynomial $q(\lambda)$ can be written in the form $q(\lambda)=q_{0}(\lambda) \cdot q_{1}(\lambda)$ and

$$
v_{0}+v_{1}=\vartheta_{0}, \quad v_{0} \cdot v_{1}=-\vartheta_{1} .
$$

The matrix $Q_{23}$ for $\vartheta_{0} \neq \vartheta_{1}$ is non-degenerate and

$$
A=Q_{23}^{-1} J_{23} Q_{23}, \quad J_{23}=\left(\begin{array}{ll}
v_{1} & 0 \\
0 & v_{0}
\end{array}\right) .
$$

Similar to [7] we can get following decomposition (45) for the process $Z(t)=$ $Q X(t)$ in the cases $\tilde{\Theta}_{2} \cup \tilde{\Theta}_{3}: Z(t)=\left(Z_{1}(t), Z_{2}(t)\right)^{\prime}, Z_{1}(t)=Q_{0} X(t)=X_{1}(t)-$ $v_{0} X_{2}(t), Z_{2}(t)=Q_{1} X(t)=X_{1}(t)-v_{1} X_{2}(t)$,

$$
\left\{\begin{array}{l}
d Z_{1}(t)=v_{1} Z_{1}(t) d t+d W(t) \\
d Z_{2}(t)=v_{0} Z_{2}(t) d t+d W(t)
\end{array}\right.
$$

This decomposition is similar to that, obtained in [14] for discrete-time autoregressive processes. 
In view of Lemma 3 in [7], putting $\varphi_{0}(T)=\mathrm{e}^{2 v_{1} T}, \varphi_{1}(T)=\mathrm{e}^{2 v_{0} T}$ in the case $\tilde{\Theta}_{2}$ and $\varphi_{0}(T)=T, \varphi_{1}(T)=\mathrm{e}^{2 v_{0} T}$ in the case $\tilde{\Theta}_{3}$ we get with $P_{\vartheta}$-probability one

$$
\lim _{T \rightarrow \infty} \bar{\varphi}^{-1 / 2}(T) Q_{23} G(T) Q_{23}^{\prime} \bar{\varphi}^{-1 / 2}(T)=F_{23},
$$

where $F_{23}$ is some positive definite matrix with random elements, which is differ in differ cases $\tilde{\Theta}_{2}$ and $\tilde{\Theta}_{3}$.

From (46) can be find the rates of increase of $\lambda_{\min }(G(T))$ and $\lambda_{\max }(G(T))$ for the cases $\tilde{\Theta}_{2}$ and $\tilde{\Theta}_{3}$, given in Table 2 .

Of course, the sign of the reals $v_{1}$ and $v_{0}$ define the asymptotic properties of the components $Z_{1}(t)$ and $Z_{2}(t)$ respectively. As we can see below, Assumption (V) is fulfilled if we put

$$
V(t)=\left(\begin{array}{cc}
1 & -\hat{v}_{0}(t) \\
1 & -\hat{v}_{1}(t)
\end{array}\right), \quad V=Q_{23}
$$

for $\vartheta \in \tilde{\Theta}_{2}$ and

$$
V(t)=\left(\begin{array}{ll}
1 & -\hat{v}_{0}(t) \\
0 & 1
\end{array}\right), \quad V=\left(\begin{array}{ll}
1 & -v_{0} \\
0 & 1
\end{array}\right)
$$

for $\tilde{\Theta}_{3}$, where

$$
\begin{gathered}
\hat{v}_{0}(t)=\log \hat{\lambda}_{0}(t), \quad \hat{v}_{1}(t)=\log \hat{\lambda}_{1}(t), \\
\hat{\lambda}_{0}(t)=\frac{\int_{0}^{t} x_{s} x_{s-1} d s}{\int_{0}^{t} x_{s-1}^{2} d s}, \quad \hat{\lambda}_{1}(t)=\frac{\int_{0}^{t} \hat{y}_{s} \hat{y}_{s-1} d s}{\int_{0}^{t} \hat{y}_{s-1}^{2} d s},
\end{gathered}
$$

$\hat{y}_{s}=x_{s}-\hat{\lambda}_{0}(s) x_{s-1}$ is an estimator of the process $y_{s}=x_{s}-\lambda_{0} x_{s-1}, s \geq 0$.

Note that we use in the case $\tilde{\Theta}_{3}$ the matrix $V$, defined in (48) instead of $Q_{23}$ because both the processes $Z_{2}(\cdot)$ and $X_{2}(\cdot)$ are unstable.

Now we define the processes $\tilde{Z}_{1}(t)=X_{1}(t)-\hat{v}_{0}(t) X_{2}(t)$ and $\tilde{Z}_{2}(t)=X_{1}(t)-$ $\hat{v}_{1}(t) X_{2}(t)$ estimating of $Z_{1}(t)$ and $Z_{2}(t)$ respectively.

Now we will find the rates of convergence for all the introduced in (48) estimators $\hat{v}_{i}(t)$ and $\hat{\lambda}_{i}(t), i=0,1$ and verify Assumptions $(\mathrm{V})$ and $(\mathrm{G})$ for the case $\tilde{\Theta}_{2}$.

From the definition of the set $\tilde{\Theta}_{2}$ it follows that the solutions $Z_{1}(t)$ and $Z_{2}(t)$ of the equations (45) have the following asymptotic properties for $t \rightarrow \infty$ :

$$
\begin{array}{ll}
\mathrm{e}^{-v_{1} t} Z_{1}(t)=Z_{1}+o\left(\mathrm{e}^{-\gamma_{1} t}\right) & P_{\vartheta}-\text { a.s.. } \\
\mathrm{e}^{-v_{0} t} Z_{2}(t)=Z_{2}+o\left(\mathrm{e}^{-\gamma_{0} t}\right) & P_{\vartheta}-\text { a.s. }
\end{array}
$$

where $0<\gamma_{1}<v_{1}, 0<\gamma_{0}<v_{0}, Z_{1}=Z_{1}(0)+\int_{0}^{\infty} \mathrm{e}^{-v_{1} s} d W(s), Z_{2}=Z_{2}(0)+$ $\int_{0}^{\infty} \mathrm{e}^{-v_{0} s} d W(s), Z_{1}(0)=\dot{x}(0)-v_{0} x(0), \quad Z_{2}(0)=\dot{x}(0)-v_{1} x(0)$.

Denote $\bar{\varphi}(T)=\operatorname{diag}\left\{\varphi_{0}(T), \varphi_{1}(T)\right\}, \varphi_{0}(T)=\mathrm{e}^{v_{1} T}, \varphi_{1}(T)=\mathrm{e}^{v_{0} T}$.

Using obtained above asymptotic properties of the functions $Z_{1}(t), Z_{2}(t)$ and the definition of $\bar{\varphi}(T)$ a short calculation shows that $P_{\vartheta}-$ a.s.

$$
\lim _{T \rightarrow \infty} \bar{\varphi}^{-1 / 2}(T) \cdot \int_{0}^{T} Z(t) Z^{\prime}(t) d t \cdot \bar{\varphi}^{-1 / 2}(T)=Z,
$$




$$
Z=\left(\begin{array}{ll}
\frac{1}{2 v_{1}} Z_{1}^{2} & \frac{1}{v_{0}+v_{1}} Z_{1} Z_{2} \\
\frac{1}{v_{0}+v_{1}} Z_{1} Z_{2} & \frac{1}{2 v_{0}} Z_{2}^{2}
\end{array}\right) .
$$

The solution of the equation (35) has the form:

$$
X(t)=\mathrm{e}^{A t} X(0)+\int_{0}^{t} \mathrm{e}^{A(t-s)} d W(s) B
$$

and, according to (44), it can be rewritten as

$$
X(t)=Q_{23}^{-1} \mathrm{e}^{J_{23} t} Q_{23} X(0)+Q_{23}^{-1} \int_{0}^{t} \mathrm{e}^{J_{23}(t-s)} d W(s) Q_{23} B .
$$

As follows, for $t \rightarrow \infty$,

$$
X_{i}(t)=C_{0 i} \mathrm{e}^{v_{0} t}+C_{1 i} \mathrm{e}^{v_{1} t}+o\left(\mathrm{e}^{\gamma_{1} t}\right),
$$

where $\gamma_{1} \in\left(0, v_{1}\right), \quad i=1,2$,

$$
\begin{gathered}
C_{0 i}=<Q_{23}^{-1} I_{1} Q_{23} X(0)+Q_{23}^{-1} I_{1} \int_{0}^{\infty} \mathrm{e}^{-J_{23} s} d W(s) Q_{23} B>_{i}, \\
C_{i}=<Q_{23}^{-1} I_{2} Q_{23} X(0)+Q_{23}^{-1} I_{2} \int_{0}^{\infty} \mathrm{e}^{-J_{23} s} d W(s) Q_{23} B>_{i}, \\
I_{1}=\left(\begin{array}{rr}
0 & 0 \\
0 & 1
\end{array}\right), \quad I_{2}=\left(\begin{array}{ll}
1 & 0 \\
0 & 0
\end{array}\right) .
\end{gathered}
$$

Then, by the definition of $\tilde{\Theta}_{2}$, as $t \rightarrow \infty$, with $P_{\vartheta}$-probability one we have

$$
\begin{aligned}
& \mathrm{e}^{-v_{0} t} X_{1}(t)=C_{01}+C_{11} \mathrm{e}^{-\left(v_{0}-v_{1}\right) t}+o\left(\mathrm{e}^{-\left(v_{0}-v_{1}-\gamma_{1}\right) t}\right), \\
& \mathrm{e}^{-v_{0} t} x_{t}=C_{02}+C_{12} \mathrm{e}^{-\left(v_{0}-v_{1}\right) t}+o\left(\mathrm{e}^{-\left(v_{0}-v_{1}-\gamma_{1}\right) t}\right), \\
& \mathrm{e}^{-v_{1} t} y_{t}=\tilde{C}_{12}+o\left(\mathrm{e}^{-\left(v_{1}-\gamma_{1}\right) t}\right),
\end{aligned}
$$

where

$$
\tilde{C}_{12}=C_{12}\left(1-\lambda_{0} \lambda_{1}^{-1}\right) .
$$

From (50), as $t \rightarrow \infty$, putting $\gamma_{2}=2\left(v_{0}-v_{1}\right) \wedge\left(v_{0}-v_{1}+\gamma_{1}\right)$, we get

$$
\begin{gathered}
\hat{\lambda}_{0}(t)-\lambda_{0}=\frac{\int_{0}^{t} x_{s-1} y_{s} d s}{\int_{0}^{t} x_{s-1}^{2} d s}=\frac{2 v_{0} \tilde{C}_{12} \mathrm{e}^{v_{0}}}{\left(v_{0}+v_{1}\right) C_{02}} \cdot \mathrm{e}^{-\left(v_{0}-v_{1}\right) t}+ \\
+\frac{v_{0} \tilde{C}_{12} C_{12} \mathrm{e}^{2 v_{0}-v_{1}}}{v_{1} C_{02}^{2}} \mathrm{e}^{-2\left(v_{0}-v_{1}\right) t}+o\left(\mathrm{e}^{-2\left(v_{0}-v_{1}\right) t}\right)+o\left(\mathrm{e}^{-\left(v_{0}-\gamma_{1}\right) t}\right) P_{\vartheta}-\text { a.s. }
\end{gathered}
$$

Then, as $t \rightarrow \infty$, with $P_{\vartheta}$-probability one we have

$$
\begin{gathered}
\hat{y}_{t}=y_{t}-\left(\hat{\lambda}_{0}(t)-\lambda_{0}\right) x_{t-1}=\frac{v_{1}-v_{0}}{v_{0}+v_{1}} \tilde{C}_{12} \mathrm{e}^{v_{1} t}+ \\
+\tilde{C}_{22} \mathrm{e}^{-\left(v_{0}-2 v_{1}\right) t}+O\left(\mathrm{e}^{-\left(2 v_{0}-3 v_{1}\right) t}\right)+o\left(\mathrm{e}^{-\gamma_{1} t}\right),
\end{gathered}
$$


where

$$
\tilde{C}_{22}=\frac{v_{0} C_{12} \tilde{C}_{12} \mathrm{e}^{v_{0}-v_{1}}}{C_{02}}\left[\frac{\mathrm{e}^{v_{0}}}{v_{1} C_{02}}+\frac{2}{v_{0}+v_{1}}\right]
$$

and denoting $\hat{C}_{22}=\tilde{C}_{22}\left(1-\lambda_{0} \lambda_{1}^{-1}\right)$, as $t \rightarrow \infty$ we obtain

$$
\hat{y}_{t}-\lambda_{1} \hat{y}_{t-1}=\hat{C}_{22} \mathrm{e}^{-\left(v_{0}-2 v_{1}\right) t}+O\left(\mathrm{e}^{-\left(2 v_{0}-3 v_{1}\right) t}\right)+o\left(\mathrm{e}^{-\gamma_{1} t}\right) \quad P_{\vartheta}-\text { a.s. }
$$

From (47), (51) and (52), as $t \rightarrow \infty$, for the estimator $\hat{\lambda}_{1}(t)$ we get

$$
\begin{aligned}
\hat{\lambda}_{1}(t)-\lambda_{1}= & \frac{\int_{0}^{t}\left(\hat{y}_{s}-\lambda_{1} \hat{y}_{s-1}\right) \hat{y}_{s-1} d s}{\int_{0}^{t} \hat{y}_{s-1}^{2} d s}=\frac{\left(v_{0}+v_{1}\right) \mathrm{e}^{v_{1}} \hat{C}_{22}}{\left(v_{1}-v_{0}\right) \tilde{C}_{12}} \cdot \mathrm{e}^{-\left(v_{0}-v_{1}\right) t}+ \\
& +O\left(\mathrm{e}^{-2\left(v_{0}-v_{1}\right) t}\right)+o\left(\mathrm{e}^{-\left(v_{1}+\gamma_{1}\right) t}\right) P_{\vartheta}-\text { a.s. }
\end{aligned}
$$

Then, by the definition (47) of the estimators $\hat{v}_{0}$ and $\hat{v}_{1}$, as $\varepsilon \rightarrow 0$ we have

$$
\begin{gathered}
\hat{v}_{0}(t)-v_{0}=C_{0 v} \mathrm{e}^{-\left(v_{0}-v_{1}\right) t}+O\left(\mathrm{e}^{-2\left(v_{0}-v_{1}\right) t}\right), \\
\hat{v}_{1}(t)-v_{1}=C_{1 v} \mathrm{e}^{-\left(v_{0}-v_{1}\right) t}+O\left(\mathrm{e}^{-2\left(v_{0}-v_{1}\right) t}\right)+o\left(\mathrm{e}^{-\left(v_{1}+\gamma_{1}\right) t}\right) \quad P_{\vartheta}-\text { a.s. },
\end{gathered}
$$

where

$$
C_{0 v}=\frac{2 v_{0} \tilde{C}_{12}}{\left(v_{0}+v_{1}\right) C_{02}}, \quad C_{1 v}=\frac{\left(v_{0}+v_{1}\right) \hat{C}_{22}}{\left(v_{1}-v_{0}\right) \tilde{C}_{12}} .
$$

From (50), (53) and (54) by the definition of $\tilde{Z}_{1}(t)=X_{1}(t)-\hat{v}_{0}(t) X_{2}(t)$ and $\tilde{Z}_{2}(t)=X_{1}(t)-\hat{v}_{1}(t) X_{2}(t)$ with $P_{\vartheta}$-probability one, for $t \rightarrow \infty$, it follows

$$
\begin{aligned}
& \mathrm{e}^{-v_{1} t} \tilde{Z}_{1}(t)=\tilde{Z}_{1}+O\left(\mathrm{e}^{-\left(v_{0}-v_{1}\right) t}\right)+o\left(\mathrm{e}^{-\gamma_{1} t}\right), \\
& \mathrm{e}^{-v_{0} t} \tilde{Z}_{2}(t)=Z_{2}+O\left(\mathrm{e}^{-\left(v_{0}-v_{1}\right) t}\right)+o\left(\mathrm{e}^{-\gamma_{0} t}\right),
\end{aligned}
$$

$\tilde{Z}_{1}=Z_{1}-C_{0} C_{0 v}$ and $P_{\vartheta}-$ a.s., as $T \rightarrow \infty$,

$$
\bar{\varphi}^{-1 / 2}(T) \cdot \int_{0}^{T} \tilde{Z}(t) \tilde{Z}^{\prime}(t) d t \cdot \bar{\varphi}^{-1 / 2}(T) \rightarrow \tilde{Z},
$$

where

$$
\tilde{Z}=\left(\begin{array}{ll}
\frac{1}{2 v_{1}} \tilde{Z}_{1}^{2} & \frac{1}{v_{0}+v_{1}} \tilde{Z}_{1} Z_{2} \\
\frac{1}{v_{0}+v_{1}} \tilde{Z}_{1} Z_{2} & \frac{1}{2 v_{0}} Z_{2}^{2}
\end{array}\right) .
$$

Then, according to (49) and (55) Assumption (V) holds true. Moreover, it can be shown by using the definition of $\bar{G}(T)$ in point 2.1 and the equation (46), that in the $P_{\vartheta}-$ a.s. sense

$$
\lim _{T \rightarrow \infty} \bar{G}^{-1}(T)=\frac{2\left(v_{0}+v_{1}\right)}{\left(v_{0}-v_{1}\right)^{2}} \cdot\left(\begin{array}{ll}
v_{1}\left(v_{0}+v_{1}\right) \tilde{Z}_{1}^{-1} Z_{1}^{-1} & -2 v_{0} v_{1} Z_{1}^{-1} \tilde{Z}_{2}^{-1} \\
-2 v_{0} v_{1} \tilde{Z}_{1}^{-1} Z_{2}^{-1} & v_{0}\left(v_{0}+v_{1}\right) Z_{2}^{-1} \tilde{Z}_{2}^{-1}
\end{array}\right) .
$$

From this it follows Assumption (G) by $\bar{g}(T) \equiv 1$ and with $P_{\vartheta}$-probability one

$$
\lim _{T: S \uparrow \infty} \tilde{G}^{-1}(S, T)=\lim _{T \rightarrow \infty} \varphi_{0}^{1 / 2}(T)\left(V^{\prime} \bar{\varphi}^{-1 / 2}(T)\right) \bar{G}^{-1}(T)=G^{\star},
$$




$$
G^{\star}=\frac{2\left(v_{0}+v_{1}\right)}{\left(v_{0}-v_{1}\right)^{2}} \cdot\left(\begin{array}{ll}
v_{1}\left(v_{0}+v_{1}\right) \tilde{Z}_{1}^{-1} Z_{1}^{-1} & -2 v_{0} v_{1} Z_{1}^{-1} \tilde{Z}_{2}^{-1} \\
-v_{0} v_{1}\left(v_{0}+v_{1}\right) \tilde{Z}_{1}^{-1} Z_{1}^{-1} & 2 v_{0}^{2} v_{1} Z_{1}^{-1} \tilde{Z}_{2}^{-1}
\end{array}\right) .
$$

Now we verify Assumptions $(\mathrm{V})$ and $(\mathrm{G})$ for the case $\tilde{\Theta}_{3}$.

In this case the solution $Z_{1}(t)=X_{1}(t)-v_{0} X_{2}(t)$ of the first equation in (45) and the solution $X(t)$ of the system (35) have the following asymptotic $P_{\vartheta}$-a.s. properties:

$$
\begin{aligned}
& \lim _{T \rightarrow \infty} \frac{1}{T} \int_{0}^{T} Z_{1}^{2}(t) d t=f_{z}, \\
& X_{i}(t)=C_{0 i} \mathrm{e}^{v_{0} t}+C_{1 i} \tilde{x}_{t}+O\left(\mathrm{e}^{v_{1} t}\right) \quad i=1,2, t \rightarrow \infty,
\end{aligned}
$$

where $\left(\tilde{x}_{t}\right)$ is a stationary process, which is a linear combination of the integrals $\int_{t}^{\infty} \mathrm{e}^{v_{0}(t-s)} d W(s)$ and $\int_{-\infty}^{t} \mathrm{e}^{v_{1}(t-s)} d W(s) ; f_{z}$ is some positive constant. As follows, with $P_{\vartheta}$-probability one,

$$
\begin{aligned}
& \lim _{T \rightarrow \infty} \frac{1}{\mathrm{e}^{2 v_{0} T}} \int_{0}^{T} x_{t}^{2} d t=\frac{C_{02}^{2}}{2 v_{0}}, \\
& y_{t}=C_{12} \tilde{x}_{t}-\lambda_{0} C_{12} \tilde{x}_{t-1}+O\left(\mathrm{e}^{v_{1} t}\right), \quad t \rightarrow \infty,
\end{aligned}
$$

where the process $y_{t}=x_{t}-\lambda_{0} x_{t-1}, \lambda_{0}=\mathrm{e}^{v_{0}}$ and for some positive constant $f_{y}$,

$$
\lim _{T \rightarrow \infty} \frac{1}{T} \int_{0}^{T} y_{t}^{2} d t=f_{y} \quad P_{\vartheta}-\text { a.s. }
$$

Define

$$
Y^{\star}(T)=\int_{-\infty}^{T} y_{t}^{\star} \mathrm{e}^{-v_{0}(T-t)} d t, \quad Y_{1}^{\star}(T)=\int_{-\infty}^{T} Z_{1}^{\star}(t) \mathrm{e}^{-v_{0}(T-t)} d t
$$

where $\left(y_{t}^{\star}\right)_{t>-\infty}$ and $\left(Z_{1}^{\star}(t)\right)_{t>-\infty}$ are stationary Gaussian processes, continuous in probability and having a spectral density, such that, as $T \rightarrow \infty$,

$$
\frac{1}{T} \int_{0}^{T}\left[y_{t}^{\star}-y_{t}\right]^{2} d t \rightarrow 0, \quad \frac{1}{T} \int_{0}^{T}\left[Z_{1}^{\star}(t)-Z_{1}(t)\right]^{2} d t \rightarrow 0 \quad P_{\vartheta}-\text { a.s. }
$$

Then the processes $Y^{\star}(T)$ and $Y_{1}^{\star}(T)$ are stationary and ergodic (see [19]) and we have with $P_{\vartheta}$-probability one:

$$
\lim _{T \rightarrow \infty}\left|\frac{1}{\mathrm{e}^{v_{0} T}} \int_{0}^{T} Z_{1}(t) \mathrm{e}^{v_{0} t} d t-Y_{1}^{\star}(T)\right|=0, \quad \lim _{T \rightarrow \infty}\left|\frac{1}{\mathrm{e}^{v_{0} T}} \int_{0}^{T} y_{t} \mathrm{e}^{v_{0} t} d t-Y^{\star}(T)\right|=0 .
$$

From (47), (57)-(60), as $t \rightarrow \infty$,

$$
\hat{\lambda}_{0}(t)-\lambda_{0}=\frac{\int_{0}^{t} x_{s} y_{s-1} d s}{\int_{0}^{t} x_{s-1}^{2} d s}=C_{0 v}(t) \cdot \mathrm{e}^{-v_{0} t}+O\left(t \mathrm{e}^{-2 v_{0} t}\right) \quad P_{\vartheta}-\text { a.s. },
$$




$$
C_{0 v}(t)=\frac{2 v_{0}}{C_{02}} Y^{\star}(t)
$$

Then, by the definition (47) of the estimators $\hat{v}_{0}$ we have

$$
\hat{v}_{0}(t)-v_{0}=C_{0 v}(t) \mathrm{e}^{-v_{0}} \cdot \mathrm{e}^{-v_{0} t}+O\left(t \mathrm{e}^{-2 v_{0} t}\right) .
$$

From $(57)$ and $(61)$ by the definition of $\tilde{Z}_{1}(t)=X_{1}(t)-\hat{v}_{0}(t) X_{2}(t)$ with $P_{\vartheta}$ probability one, as $t \rightarrow \infty$, it follows

$$
\tilde{Z}_{1}(t)=Z_{1}^{\star \star}(t)+\Delta Z_{1}(t)+O\left(t \mathrm{e}^{-v_{0} t}\right)
$$

$Z_{1}^{\star \star}(t)=Z_{1}^{\star}(t)-2 v_{0} Y^{\star}(t), \Delta Z_{1}(t)=Z_{1}(t)-Z_{1}^{\star}(t)$ and denoting

$$
Z^{\star \star}(T)=\int_{-\infty}^{T} Z_{1}^{\star \star}(t) \mathrm{e}^{-v_{0}(T-t)} d t
$$

we obtain for stationary process $\left(Z^{\star \star}(T)\right)$ with $P_{\vartheta}$-probability one the following properties of the process $\left(\tilde{Z}_{1}(t)\right)$ :

$$
\lim _{T \rightarrow \infty}\left|\frac{1}{\mathrm{e}^{v_{0} T}} \int_{0}^{T} \tilde{Z}_{1}(t) \mathrm{e}^{v_{0} t} d t-Z^{\star \star}(T)\right|=0, \quad \lim _{T \rightarrow \infty} \frac{1}{T} \int_{0}^{T} \tilde{Z}_{1}^{2}(t) d t=E_{\vartheta}\left(Z_{1}^{\star \star}(0)\right)^{2} .
$$

From $(57),(60)$ and $(62)$ we find with $P_{\vartheta}$-probability one:

$$
\begin{aligned}
& \lim _{T \rightarrow \infty}\left|\frac{1}{\mathrm{e}^{v_{0} T}} \int_{0}^{T} Z_{1}(t) x_{t} d t-C_{02} Y_{1}^{\star}(T)\right|=0, \\
& \lim _{T \rightarrow \infty}\left|\frac{1}{\mathrm{e}^{v_{0} T}} \int_{0}^{T} \tilde{Z}_{1}(t) x_{t} d t-C_{02} Z^{\star \star}(T)\right|=0 .
\end{aligned}
$$

By the definition of the processes $Y_{1}^{\star}(T)$ and $Z^{\star \star}(T)$ it immediately follows, that for $h \rightarrow 0$ we have $E\left[Y_{1}^{\star}(T+h)-Y_{1}^{\star}(T)\right]^{2}=O(h)$ and $E\left[Z^{\star \star}(T+h)-Z^{\star \star}(T)\right]^{2}=O(h)$. Then, from Proposition 2 in the Appendix it follows that $\left|Y_{1}^{\star}(T)\right|=O(\sqrt{\ln T})$, $\left|Z^{\star \star}(T)\right|=O(\sqrt{\ln T})$, as $T \rightarrow \infty P_{\vartheta}-$ a.s. Then, putting $b(t)=\left(\tilde{Z}_{1}(t), x_{t}\right)^{\prime}, \bar{b}(t)=$ $\left(Z_{1}(t), x_{t}\right)^{\prime}, \varphi_{0}(T)=T, \varphi_{1}(T)=\mathrm{e}^{2 v_{0} T}$, we can find the $P_{\vartheta}-$ a.s. - limit

$$
\lim _{T \rightarrow \infty} \bar{G}(T)=\left(\begin{array}{ll}
E_{\vartheta} Z_{1}^{\star}(0) Z_{1}^{\star \star}(0) & 0 \\
0 & \frac{C_{02}^{2}}{2 v_{0}}
\end{array}\right) .
$$

Thus for $\alpha=v_{0}, \Psi_{1}(\alpha, x)=\mathrm{e}^{2 v_{0} x}$, Assumptions (V) and (G) hold true and, similarly to (56), with $P_{\vartheta}$-probability one,

$$
\begin{gathered}
\lim _{T: S \uparrow \infty} \tilde{G}^{-1}(S, \bar{T})=\tilde{G}_{3}, \\
\tilde{G}_{3}=\left[E_{\vartheta} Z_{1}^{\star}(0) Z_{1}^{\star \star}(0)\right]^{-1} \cdot\left(\begin{array}{ll}
1 & 0 \\
-v_{0} & 0
\end{array}\right) .
\end{gathered}
$$

Thus all the Assumptions (V), $(\mathrm{G}),(\varphi \Psi)$ and $(\Psi)$ are fulfilled if we put the set $\mathcal{A}=(0,+\infty)$ in the cases $\tilde{\Theta}_{12} \cup \tilde{\Theta}_{14} \cup \tilde{\Theta}_{3}, \mathcal{A}=\left\{\left(v_{0}, v_{1}\right): 0<v_{1}<v_{0}<\infty\right\}$ in the case $\tilde{\Theta}_{2}$ and 
Table 2

\begin{tabular}{|c||c|c||c|c|}
\hline Region & $b_{0}(t)$ & $b_{1}(T)$ & $\bar{b}_{0}(t)$ & $\bar{b}_{1}(T)$ \\
\hline$\tilde{\Theta}_{11}$ & $X_{1}(t)$ & $X_{2}(t)$ & $X_{1}(t)$ & $X_{2}(t)$ \\
\hline$\tilde{\Theta}_{12}$ & $X_{1}(t)$ & $X_{2}(t)$ & $X_{1}(t)$ & $X_{2}(t)$ \\
\hline$\tilde{\Theta}_{13}$ & $X_{1}(t)$ & $X_{2}(t)$ & $X_{1}(t)$ & $X_{2}(t)$ \\
\hline$\tilde{\Theta}_{14}$ & $X_{1}(t)$ & $X_{2}(t)$ & $X_{1}(t)$ & $X_{2}(t)$ \\
\hline$\tilde{\Theta}_{2}$ & $\tilde{Z}_{1}(t)$ & $\tilde{Z}_{2}(t)$ & $Z_{1}(t)$ & $Z_{2}(t)$ \\
\hline$\tilde{\Theta}_{3}$ & $\tilde{Z}_{1}(t)$ & $X_{2}(t)$ & $Z_{1}(t)$ & $X_{2}(t)$ \\
\hline
\end{tabular}

Table 3

\begin{tabular}{|l||l|l||l|l|l|}
\hline Region & $\varphi_{0}(\alpha, T)$ & $\varphi_{1}(\alpha, T)$ & $\alpha$ & $\Psi_{1}(\alpha, x)$ & $g(\varphi)$ \\
\hline$\tilde{\Theta}_{11}$ & $T$ & $T$ & - & $x$ & 1 \\
\hline$\tilde{\Theta}_{12}$ & $e^{2 v_{0} T}$ & $e^{2 v_{0} T}$ & $v_{0}$ & $x$ & 1 \\
\hline$\tilde{\Theta}_{13}$ & $T^{2}$ & $T^{2}$ & - & $x$ & 1 \\
\hline$\tilde{\Theta}_{14}$ & $T^{2} e^{2 v_{0} T}$ & $T^{2} e^{2 v_{0} T}$ & - & $x$ & $\ln ^{8} \varphi$ \\
\hline$\tilde{\Theta}_{2}$ & $e^{2 v_{1} T}$ & $e^{2 v_{0} T}$ & $\left(v_{0}, v_{1}\right)$ & $x^{v_{0} / v_{1}}$ & 1 \\
\hline$\tilde{\Theta}_{3}$ & $T$ & $e^{2 v_{0} T}$ & $v_{0}$ & $e^{2 v_{0} x}$ & 1 \\
\hline
\end{tabular}

Then $l_{0}=2, m=0$ for $\vartheta \in \tilde{\Theta}_{1} \cup \tilde{\Theta}_{2}$ and $l_{0}=1, l_{1}=1, m=1$ for $\vartheta \in \tilde{\Theta}_{3}$.

Further we shall construct sequential estimation plans in all cases $\tilde{\Theta}_{1}, \tilde{\Theta}_{2}$ and $\tilde{\Theta}_{3}$ separately. Then we shall use these plans for the construction of the sequential estimator, which works on the whole plane $R^{2}$ except of the set $\left\{\vartheta \in R^{2}: \vartheta_{1}=0\right\}$.

\subsection{Estimation procedure for the case $\tilde{\Theta}_{1}$}

From Table 3 it is clear, that the functions $\varphi_{0}(T)$ and $\varphi_{1}(T)$ are equal if $\vartheta$ varies in $\tilde{\Theta}_{1 i}, i=\overline{1,4}$. They are different for $\vartheta$ from different $\tilde{\Theta}_{1 i}$. Moreover, in all these subsets we have $V(t) \equiv V=I$. Thus we can define a sequential estimation plan of the type (20) for all set $\tilde{\Theta}_{1}$, where $\nu_{n}(\varepsilon)=0, n \geq 1$ and in the definition of stopping times $\tau_{0}(n, \varepsilon)$ we take $\Psi_{1}\left(\alpha(n, \varepsilon), \varepsilon^{-1} c_{n}\right)=\varepsilon^{-1} c_{n}$.

Denote SEP1 $(\varepsilon)=\left(T_{1}(\varepsilon), \vartheta_{1}(\varepsilon)\right)$ the sequential plan $(20)$ with these parameters, which in the considered case has the form:

$$
T_{1}(\varepsilon)=\tau_{1}\left(\sigma_{1}(\varepsilon), \varepsilon\right), \quad \vartheta_{1}(\varepsilon)=S_{1}^{-1}\left(\sigma_{1}(\varepsilon), \varepsilon\right) \sum_{n=1}^{\sigma_{1}(\varepsilon)} \beta_{1}^{q}(n, \varepsilon) \vartheta_{1}(n, \varepsilon),
$$

where

$$
\begin{gathered}
\tau_{1}(n, \varepsilon)=\inf \left\{T>0:\left(\int_{0}^{T} X_{1}^{2}(t) d t\right)^{q / 2}+\left(\int_{0}^{T} X_{2}^{2}(t) d t\right)^{q / 2}=\left(\varepsilon^{-1} c_{n}\right)^{q / 2}\right\}, \\
\sigma_{1}(\varepsilon)=\inf \left\{N \geq 1: S_{1}(N, \varepsilon) \geq \delta_{1}^{-1} \varrho_{1}\right\},
\end{gathered}
$$

$\delta_{1} \in(0,1)$ is an arbitrary chosen constant and $\varrho_{1}=b_{q} 2^{\frac{q-2}{q}} \sum_{n \geq 1} c_{n}^{-q / 2}$;

$S_{1}(N, \varepsilon)=\sum_{n=1}^{N} \beta_{1}^{q}(n, \varepsilon), \beta_{1}(n, \varepsilon)=\left\|G_{1}^{-1}(n, \varepsilon)\right\|^{-1}, \quad G_{1}(n, \varepsilon)=\left(\varepsilon^{-1} c_{n}\right)^{-1} G\left(\tau_{1}(n, \varepsilon)\right)$, 


$$
\begin{gathered}
G(T)=\int_{0}^{T} X(t) X^{\prime}(t) d t, \quad X(t)=\left(\dot{x}_{t}, x_{t}\right)^{\prime} ; \\
\vartheta_{1}(n, \varepsilon)=G^{-1}\left(\tau_{1}(n, \varepsilon)\right) \cdot \Phi\left(\tau_{1}(n, \varepsilon)\right), \quad \Phi(T)=\int_{0}^{T} X(t) d X_{1}(t) .
\end{gathered}
$$

Now we introduce the following notation: $[a]_{1}$ is the integer part of $a ; a \wedge$ $b=\min (\mathrm{a}, \mathrm{b}), a \vee b=\max (\mathrm{a}, \mathrm{b})$;

$$
\begin{aligned}
& f_{11}=\left[<F_{11}>_{11}^{q / 2}+<F_{11}>_{22}^{q / 2}\right]^{2 / q}, \quad \Delta_{11}=f_{11} \cdot\left\|F_{11}^{-1}\right\|, \\
& r_{11}^{\prime}=f_{11}^{-1} \cdot c_{\left[\delta_{1}^{-1} \Delta_{11}^{q} \varrho_{1}-1\right]_{1} \vee 1}, \quad r_{11}^{\prime \prime}=f_{11}^{-1} \cdot c_{\left[\delta_{1}^{-1} \Delta_{11}^{q} \varrho_{1}\right]_{1}+1} ; \\
& f_{12}^{\prime}=\left[\sup _{T>0}\left(<F_{12}(T)>_{11}^{q / 2}+<F_{12}(T)>_{22}^{q / 2}\right)\right]^{2 / q}, \\
& f_{12}^{\prime \prime}=\left[\inf _{T>0}\left(<F_{12}(T)>_{11}^{q / 2}+<F_{12}(T)>_{22}^{q / 2}\right)\right]^{2 / q}, \\
& \Delta_{12}^{\prime}=f_{12}^{\prime \prime} \cdot \inf _{T>0}\left\|F_{12}^{-1}(T)\right\|, \quad \Delta_{12}^{\prime \prime}=f_{12}^{\prime} \cdot \sup _{T>0}\left\|F_{12}^{-1}(T)\right\|, \\
& r_{12}^{\prime}=\frac{1}{2 v_{0}} \ln \left(f_{12}^{\prime}\right)^{-1} \cdot c_{\left[\delta_{1}^{-1} \rho_{1}\left(\Delta_{12}^{\prime}\right)^{q}-1\right]_{1} \vee 1}, \quad r_{12}^{\prime \prime}=\frac{1}{2 v_{0}} \ln \left(f_{12}^{\prime \prime}\right)^{-1} \cdot c_{\left[\delta_{1}^{-1} \rho_{1}\left(\Delta_{12}^{\prime \prime}\right)^{q}\right]_{1}+1} ; \\
& f_{13}=\left[<F_{13}>_{11}^{q / 2}+<F_{13}>_{22}^{q / 2}\right]^{2 / q}, \quad \Delta_{13}=f_{13}\left\|F_{13}^{-1}\right\|, \\
& r_{13}^{\prime}=\sqrt{f_{13}^{-1} c_{\left[\delta_{1}^{-1} \rho_{1} \Delta_{13}^{q}-1\right]_{1} \vee 1}}, \quad r_{13}^{\prime \prime}=\sqrt{f_{13}^{-1} c_{\left[\delta_{1}^{-1} \rho_{1} \Delta_{13}^{q}+1\right]_{1}}} ; \\
& f_{14}=\left[<F_{14}>_{11}^{q / 2}+<F_{14}>_{22}^{q / 2}\right]^{2 / q}, \quad \Delta_{14}=f_{14}\left(v_{0}^{4}\left|F_{14}\right|\right)^{-1}\left\|F_{14}\right\|, \\
& \sigma_{14}(\varepsilon)=\left[\delta_{1}^{-1} \varrho_{1} v_{0}^{-4 q} \Delta_{14}^{q} \ln ^{4 q} \varepsilon^{-1}\right]_{1}+1, \\
& r_{14}^{\prime}=\frac{1}{2 v_{0}} \ln f_{14}^{-1} c_{\left[\delta_{1}^{-1} \rho_{1} \Delta_{14}^{q}-1\right]_{1} \vee 1}, \quad r_{14}^{\prime \prime}=\frac{1}{2 v_{0}} \ln f_{14}^{-1} .
\end{aligned}
$$

The next corollary concentrate the basic properties of the considered estimators.

Corollary 3.1 Let the parameter $\vartheta$ in (34) belongs to the set $\tilde{\Theta}_{1}$. Then for any $\varepsilon>0$ the sequential plan $\mathrm{SEP} 1(\varepsilon)$ defined in $(64)$ is closed. It has the following properties:

$1^{\circ}$. for any $\varepsilon>0$

$$
\sup _{\vartheta \in \tilde{\Theta}_{1}}\left\|\vartheta_{1}(\varepsilon)-\vartheta\right\|_{q}^{2} \leq \varepsilon \delta_{1}
$$

$2^{\circ}$. the following relations hold with $P_{\vartheta}-$ probability one:

- for $\vartheta \in \tilde{\Theta}_{11}$

$$
0<r_{11}^{\prime} \leq \lim _{\varepsilon \rightarrow 0} \varepsilon T_{1}(\varepsilon) \leq \varlimsup_{\varepsilon \rightarrow 0} \varepsilon T_{1}(\varepsilon) \leq r_{11}^{\prime \prime}<\infty,
$$

- for $\vartheta \in \tilde{\Theta}_{12}$

$$
0<r_{12}^{\prime} \leq \lim _{\varepsilon \rightarrow 0}\left[T_{1}(\varepsilon)-\frac{1}{2 v_{0}} \ln \varepsilon^{-1}\right] \leq \varlimsup_{\varepsilon \rightarrow 0}\left[T_{1}(\varepsilon)-\frac{1}{2 v_{0}} \ln \varepsilon^{-1}\right] \leq r_{12}^{\prime \prime}<\infty,
$$


- for $\vartheta \in \tilde{\Theta}_{13}$

$$
0<r_{13}^{\prime} \leq \varliminf_{\varepsilon \rightarrow 0} \sqrt{\varepsilon} T_{1}(\varepsilon) \leq \varlimsup_{\varepsilon \rightarrow 0} \sqrt{\varepsilon} T_{1}(\varepsilon) \leq r_{13}^{\prime \prime}<\infty,
$$

- for $\vartheta \in \tilde{\Theta}_{14}$

$$
\begin{gathered}
\lim _{\varepsilon \rightarrow 0}\left[T_{1}(\varepsilon)+\frac{1}{v_{0}} \ln T_{1}(\varepsilon)-\frac{1}{2 v_{0}} \ln \varepsilon^{-1}\right] \geq r_{14}^{\prime}>0, \\
\varlimsup_{\varepsilon \rightarrow 0}\left[T_{1}(\varepsilon)+\frac{1}{v_{0}} \ln T_{1}(\varepsilon)-\frac{1}{2 v_{0}} \ln \varepsilon^{-1}-\frac{1}{2 v_{0}} \ln c_{\sigma_{14}(\varepsilon)}\right] \leq r_{14}^{\prime \prime}<\infty ;
\end{gathered}
$$

$3^{\circ}$. the estimator $\vartheta_{1}(\varepsilon)$ is strongly consistent:

$$
\lim _{\varepsilon \rightarrow 0} \vartheta_{1}(\varepsilon)=\vartheta \quad P_{\vartheta}-\text { a.s. }
$$

Proof. The proof of Corollary 3.1, except of the second assertion, follows directly from Theorem 1 . Now we verify assertion $2^{\circ}$.

From $(36),(39),(41)-(43)$ and the definitions of $\tau_{1}(n, \varepsilon)$ and $\beta_{1}(n, \varepsilon)$ it follows with $P_{\vartheta}$-probability one: for $n \geq 1$

- for $\vartheta \in \tilde{\Theta}_{11}$

$$
\lim _{\varepsilon \rightarrow 0} \varepsilon \tau_{1}(n, \varepsilon)=f_{11}^{-1} \cdot c_{n}, \quad \lim _{\varepsilon \rightarrow 0} \beta_{1}(n, \varepsilon)=\Delta_{11}^{-1},
$$

- for $\vartheta \in \tilde{\Theta}_{12}$

$$
\begin{aligned}
\left(f_{12}^{\prime}\right)^{-1} \cdot c_{n} & \leq \varliminf_{\varepsilon \rightarrow 0} \varepsilon \mathrm{e}^{\tau_{1}(n, \varepsilon)} \leq \varlimsup_{\varepsilon \rightarrow 0} \varepsilon \mathrm{e}^{\tau_{1}(n, \varepsilon)} \leq\left(f_{12}^{\prime \prime}\right)^{-1} \cdot c_{n}, \\
\left(\Delta_{12}^{\prime}\right)^{-1} & \leq \varliminf_{\varepsilon \rightarrow 0} \beta_{1}(n, \varepsilon) \leq \varlimsup_{\varepsilon \rightarrow 0} \beta_{1}(n, \varepsilon) \leq\left(\Delta_{12}^{\prime \prime}\right)^{-1},
\end{aligned}
$$

- for $\vartheta \in \tilde{\Theta}_{13}$

$$
\lim _{\varepsilon \rightarrow 0} \sqrt{\varepsilon} \tau_{1}(n, \varepsilon)=\sqrt{f_{13}^{-1} \cdot c_{n}}, \quad \lim _{\varepsilon \rightarrow 0} \beta_{1}(n, \varepsilon)=\Delta_{13}^{-1},
$$

- for $\vartheta \in \tilde{\Theta}_{14}$

$$
\begin{aligned}
& \lim _{\varepsilon \rightarrow 0}\left[\tau_{1}(n, \varepsilon)+\frac{1}{v_{0}} \ln \tau_{1}(n, \varepsilon)-\frac{1}{2 v_{0}} \ln \varepsilon^{-1}\right]=\frac{1}{2 v_{0}} \ln f_{14}^{-1}+\frac{1}{2 v_{0}} \ln c_{n}, \\
& \left(2 v_{0}\right)^{4} \Delta_{14}^{-1} \leq \lim _{\varepsilon \rightarrow 0} \ln ^{4} \varepsilon^{-1} \cdot \beta_{1}(n, \varepsilon), \quad \varlimsup_{\varepsilon \rightarrow 0} \beta_{1}(n, \varepsilon) \leq \Delta_{14}^{-1}
\end{aligned}
$$

Then by the definition of $\sigma_{1}(\varepsilon)$ we have:

- for $\vartheta \in \tilde{\Theta}_{11}$

$\left(\left[\delta_{1}^{-1} \Delta_{11}^{q} \varrho_{1}\right]_{1}-1\right) \vee 1 \leq \lim _{\varepsilon \rightarrow 0} \sigma_{1}(\varepsilon) \leq \varlimsup_{\varepsilon \rightarrow 0} \sigma_{1}(\varepsilon) \leq\left[\delta_{1}^{-1} \Delta_{11}^{q} \varrho_{1}\right]_{1}+1 \quad P_{\vartheta}-$ a.s.,

- for $\vartheta \in \tilde{\Theta}_{12}$

$$
\left[\delta_{1}^{-1} \varrho_{1} \Delta_{12}^{q}-1\right]_{1} \vee 1 \leq \lim _{\varepsilon \rightarrow 0} \sigma_{1}(\varepsilon) \leq \varlimsup_{\varepsilon \rightarrow 0} \sigma_{1}(\varepsilon) \leq\left[\delta_{1}^{-1} \varrho_{1} \Delta_{12}^{q}\right]_{1}+1 \quad P_{\vartheta}-\text { a.s. }
$$


- for $\vartheta \in \tilde{\Theta}_{13}$

$\left(\left[\delta_{1}^{-1} \varrho_{1} \Delta_{13}^{q}\right]_{1}-1\right) \vee 1 \leq \lim _{\varepsilon \rightarrow 0} \sigma_{1}(\varepsilon) \leq \varlimsup_{\varepsilon \rightarrow 0} \sigma_{1}(\varepsilon) \leq\left[\delta_{1}^{-1} \varrho_{1} \Delta_{13}^{q}\right]_{1}+1 \quad P_{\vartheta}-$ a.s.,

- for $\vartheta \in \tilde{\Theta}_{14}$

$$
\left(\left[\delta_{1}^{-1} \varrho_{1} \Delta_{14}^{q}\right]_{1}-1\right) \vee 1 \leq \lim _{\varepsilon \rightarrow 0} \sigma_{1}(\varepsilon) \quad P_{\vartheta}-\text { a.s. }
$$

and for $\varepsilon$ small enough

$$
\sigma_{1}(\varepsilon) \leq \sigma_{14}(\varepsilon) \quad P_{\vartheta}-\text { a.s. }
$$

From the definition of $T_{1}(\varepsilon)$ we finally get the second assertion of Corollary 3.1.

Hence Corollary 3.1 is proved.

Remark 3.2. Similar to [6], the asymptotic constants $r_{11}^{\prime}$ and $r_{11}^{\prime \prime}$ in the stationary case $\tilde{\Theta}_{11}$ can be changed by $r_{11}^{\prime}=r_{11}^{\prime \prime}=f_{11}^{-1}$ (it coincides with the optimal convergence rate of the MLE) by appropriate chosen sequences $\left(c_{n}\right)$ and $\left(\kappa_{n}\right)$.

If in the case $\tilde{\Theta}_{14}, c_{n}=o\left(\mathrm{e}^{\left(n^{a}\right)}\right)$ as $n \rightarrow \infty, a=1 / 4 q$, then $\ln c_{\sigma_{14}(\varepsilon)}=o\left(\ln \varepsilon^{-1}\right)$, as $\varepsilon \rightarrow 0$ and

$$
\lim _{\varepsilon \rightarrow 0} \frac{T_{1}(\varepsilon)}{\ln \varepsilon^{-1}}=\frac{1}{2 v_{0}} \quad P_{\vartheta}-\text { a.s. }
$$

\subsection{Estimation procedure for the case $\tilde{\Theta}_{2}$}

Now we define the sequential plan $\operatorname{SEP} 2(\varepsilon)$ for estimation $\vartheta \in \tilde{\Theta}_{2}$.

Put

$$
\nu_{n}(\varepsilon)=\frac{\ln \varepsilon^{-1} c_{n}}{\ln \ln \varepsilon^{-1} c_{n}}, n \geq 1, \varepsilon>0
$$

and in the definition of the stopping times $\tau(n, \varepsilon)$ we take

$$
\begin{gathered}
\alpha(n, \varepsilon):=\alpha_{2}(n, \varepsilon)=\left(\hat{v}_{1}\left(\nu_{n}(\varepsilon)\right), \hat{v}_{0}\left(\nu_{n}(\varepsilon)\right),\right. \\
\Psi_{1}\left(\tilde{\alpha}_{2}(n, \varepsilon), \varepsilon^{-1} c_{n}\right)=\left(\varepsilon^{-1} c_{n}\right)^{\tilde{\alpha}_{2}(n, \varepsilon)}, \\
\tilde{\alpha}_{2}(n, \varepsilon)=\hat{v}_{0}\left(\nu_{n}(\varepsilon)\right) / \hat{v}_{1}\left(\nu_{n}(\varepsilon)\right),
\end{gathered}
$$

where $\hat{v}_{0}(\cdot)$ and $\hat{v}_{1}(\cdot)$ are defined in $(47)$.

Therefore, using (53) and (54) a short calculation gives for $i=1,2$ the equalities

$$
\lim _{n \vee \varepsilon} \frac{\tilde{\Psi}_{i i}(n, \varepsilon)}{\Psi_{i i}(\alpha, n, \varepsilon)}=1, \quad P_{\vartheta}-\text { a.s. }
$$

and, as follows, Assumptions $(\alpha 1)$ and $(\alpha 2)$ are fulfilled. Thus all conditions of Theorem 1 hold true.

Denote SEP2 $(\varepsilon)=\left(T_{2}(\varepsilon), \vartheta_{2}(\varepsilon)\right)$ the sequential plan $(20)$ with these parameters, which in considered case has the form:

$$
T_{2}(\varepsilon)=\tau_{2}\left(\sigma_{2}(\varepsilon), \varepsilon\right), \quad \vartheta_{2}(\varepsilon)=S_{2}^{-1}\left(\sigma_{2}(\varepsilon), \varepsilon\right) \sum_{n=1}^{\sigma_{2}(\varepsilon)} \beta_{2}^{q}(n, \varepsilon) \vartheta_{2}(n, \varepsilon),
$$


where

$$
\begin{gathered}
\tau_{2}(n, \varepsilon)=\inf \left\{T>0:\left(\frac{1}{\varepsilon^{-1} c_{n}} \int_{\nu_{n}(\varepsilon)}^{T} \tilde{Z}_{1}^{2}(t) d t\right)^{q / 2}+\right. \\
\left.+\left(\frac{1}{\left(\varepsilon^{-1} c_{n}\right)^{\tilde{\alpha}_{2}(n, \varepsilon)}} \int_{\nu_{n}(\varepsilon)}^{T} \tilde{Z}_{2}^{2}(t) d t\right)^{q / 2}=1\right\}, \\
\sigma_{2}(\varepsilon)=\inf \left\{N \geq 1: S_{2}(N, \varepsilon) \geq \delta_{2}^{-1} \varrho_{1}\right\},
\end{gathered}
$$

$\delta_{2} \in(0,1)$ is some arbitrary chosen constant;

$$
\begin{gathered}
S_{2}(N, \varepsilon)=\sum_{n=1}^{N} \beta_{2}^{q}(n, \varepsilon), \beta_{2}(n, \varepsilon)=\left\|G_{2}^{-1}(n, \varepsilon)\right\|^{-1}, \\
G_{2}(n, \varepsilon)=\left(\varepsilon^{-1} c_{n}\right)^{-1 / 2} \tilde{\Psi}^{-1 / 2}(n, \varepsilon) G\left(\nu_{n}(\varepsilon), \tau_{2}(n, \varepsilon)\right), \\
\tilde{\Psi}(n, \varepsilon)=\operatorname{diag}\left\{\varepsilon^{-1} c_{n},\left(\varepsilon^{-1} c_{n}\right)^{\tilde{\alpha}_{2}(n, \varepsilon)}\right\}, \\
G(S, T)=\int_{S}^{T} \tilde{Z}(t) X^{\prime}(t) d t, \tilde{Z}(t)=\left(\tilde{Z}_{1}(t), \tilde{Z}_{2}(t)\right)^{\prime}, \\
\vartheta_{2}(n, \varepsilon)=G^{-1}\left(\nu_{n}(\varepsilon), \tau_{2}(n, \varepsilon)\right) \cdot \Phi\left(\nu_{n}(\varepsilon), \tau_{2}(n, \varepsilon)\right), \\
\Phi(S, T)=\int_{S}^{T} \tilde{Z}(t) d X_{1}(t) .
\end{gathered}
$$

Now we introduce following notation: let $s_{q}$ be the positive root of the equation

$$
\begin{gathered}
\left(s_{q} \cdot \frac{\tilde{Z}_{1}^{2}}{2 v_{1}}\right)^{q / 2}+\left(s_{q}^{v_{0} / v_{1}} \cdot \frac{Z_{2}^{2}}{2 v_{0}}\right)^{q / 2}=1, \\
S^{\star}=\operatorname{diag}\left\{s_{q}^{-1}, s_{q}^{-\frac{v_{0}+v_{1}}{2 v_{1}}}\right\}, \quad \Delta_{2}=\left\|G^{\star} \cdot S^{\star}\right\|
\end{gathered}
$$

and define

$$
r_{2}^{\prime}=\frac{1}{2 v_{1}} \ln s_{q} \cdot c_{\left[\delta_{2}^{-1} \varrho_{1} \Delta_{2}^{q}-1\right]_{1} \vee 1}, \quad r_{2}^{\prime \prime}=\frac{1}{2 v_{1}} \ln s_{q} \cdot c_{\left[\delta_{2}^{-1} \varrho_{1} \Delta_{2}^{q}\right]_{1}+1} .
$$

Corollary 3.2 Let the parameter $\vartheta$ in (34) belongs to the set $\tilde{\Theta}_{2}$. Then for any $\varepsilon>0$ the sequential plan SEP2 $(\varepsilon)$ defined in $(65)$ is closed. It has the following properties: $1^{\circ}$. for any $\varepsilon>0$

$$
\sup _{\vartheta \in \tilde{\Theta}_{2}}\left\|\vartheta_{2}(\varepsilon)-\vartheta\right\|_{q}^{2} \leq \varepsilon \delta_{2}
$$

$2^{\circ}$. the following relations hold with $P_{\vartheta}-$ probability one:

$$
0<r_{2}^{\prime} \leq \varliminf_{\varepsilon \rightarrow 0}\left[T_{2}(\varepsilon)-\frac{1}{2 v_{1}} \ln \varepsilon^{-1}\right] \leq \varlimsup_{\varepsilon \rightarrow 0}\left[T_{2}(\varepsilon)-\frac{1}{2 v_{1}} \ln \varepsilon^{-1}\right] \leq r_{2}^{\prime \prime}<\infty ;
$$


$3^{\circ}$. the estimator $\vartheta_{2}(\varepsilon)$ is strongly consistent:

$$
\lim _{\varepsilon \rightarrow 0} \vartheta_{2}(\varepsilon)=\vartheta \quad P_{\vartheta}-\text { a.s. }
$$

Proof. The proof of Corollary 3.2, except of second assertion, follows from Theorem 1. Assertion $2^{\circ}$ can be verified similar to the second assertion of Corollary 3.1. Indeed, from the definition (65) of stopping times $\tau_{2}(n, \varepsilon)$ and $(53)-(56)$ we can find the limits

$$
\begin{gathered}
\lim _{n \vee \varepsilon} \frac{\mathrm{e}^{2 v_{1} \tau_{2}(n, \varepsilon)}}{\varepsilon^{-1} c_{n}}=s_{q} \quad P_{\vartheta}-\text { a.s. } \\
\lim _{n \vee \varepsilon} G_{2}^{-1}(n, \varepsilon)=G^{\star} \cdot S^{\star} \quad P_{\vartheta}-\text { a.s. }
\end{gathered}
$$

and, by the definition of $\beta_{2}(n, \varepsilon)$ :

$$
\lim _{n \vee \varepsilon} \beta_{2}(n, \varepsilon)=\Delta_{2}^{-1} \quad P_{\vartheta}-\text { a.s. }
$$

The second assertion of Corollary 3.2 follows from (66)-(68).

The proof of Corollary 3.2 is complete.

\subsection{Estimation procedure for the case $\tilde{\Theta}_{3}$}

Let $\left(\nu_{n}(\varepsilon)\right)_{n \geq 1}, \varepsilon>0$ be some chosen non-random function, satisfying the condition (14) and such that

$$
\lim _{n \vee \varepsilon} \frac{\nu_{n}(\varepsilon)}{\ln \varepsilon^{-1} c_{n}}=\infty
$$

In the definition of stopping times $\tau(n, \varepsilon)$ we take $\alpha(n, \varepsilon):=\alpha_{3}(n, \varepsilon)=\hat{v}_{0}\left(\nu_{n}(\varepsilon)\right)$, $\Psi_{1}\left(\alpha(n, \varepsilon), \varepsilon^{-1} c_{n}\right)=\mathrm{e}^{2 \hat{v}_{0}\left(\nu_{n}(\varepsilon)\right) \varepsilon^{-1} c_{n}}$, where $\hat{v}_{0}(\cdot)$ is defined in $(48)$.

Therefore, the Assumptions $(\alpha 1)$ and $(\alpha 2)$ are fulfilled, because according to (61) and (69)

$$
\lim _{n \vee \varepsilon} \frac{\tilde{\Psi}_{22}(n, \varepsilon)}{\Psi_{22}(\alpha, n, \varepsilon)}=\lim _{n \vee \varepsilon} \mathrm{e}^{2\left(\hat{v}_{0}\left(\nu_{n}(\varepsilon)\right)-v_{0}\right) \varepsilon^{-1} c_{n}}=1 \quad P_{\vartheta}-\text { a.s. }
$$

and therefore all conditions of Theorem 1 hold true.

Denote SEP3 $(\varepsilon)=\left(T_{3}(\varepsilon), \vartheta_{3}(\varepsilon)\right)$ the sequential plan $(20)$ with these parameters, which in considered case has the form:

$$
\begin{aligned}
& T_{3}(\varepsilon)=\max \left\{\tau_{31}\left(\sigma_{3}(\varepsilon), \varepsilon\right), \tau_{32}\left(\sigma_{3}(\varepsilon), \varepsilon\right)\right\} \\
& \vartheta_{3}(\varepsilon)=S_{3}^{-1}\left(\sigma_{3}(\varepsilon), \varepsilon\right) \sum_{n=1}^{\sigma_{3}(\varepsilon)} \beta_{3}^{q}(n, \varepsilon) \vartheta_{3}(n, \varepsilon)
\end{aligned}
$$

where

$$
\tau_{31}(n, \varepsilon)=\inf \left\{T>0: \int_{\nu_{n}(\varepsilon)}^{T} \tilde{Z}_{1}^{2}(t) d t=\varepsilon^{-1} c_{n}\right\}
$$




$$
\begin{gathered}
\tau_{32}(n, \varepsilon)=\inf \left\{T>0: \int_{\nu_{n}(\varepsilon)}^{T} x_{t}^{2} d t=\mathrm{e}^{2 \hat{v}_{0}\left(\nu_{n}(\varepsilon)\right) \varepsilon^{-1} c_{n}}\right\}, \\
\sigma_{3}(\varepsilon)=\inf \left\{N \geq 1: S_{3}(N, \varepsilon) \geq 2 \delta_{3}^{-1} \varrho_{1}\right\},
\end{gathered}
$$

$\delta_{3} \in(0,1)$ is some arbitrary chosen constant;

$$
\begin{gathered}
S_{3}(N, \varepsilon)=\sum_{n=1}^{N} \beta_{3}^{q}(n, \varepsilon), \beta_{3}(n, \varepsilon)=\left\|G_{3}^{-1}(n, \varepsilon)\right\|^{-1}, \\
\tau_{\min }(n, \varepsilon)=\min \left\{\tau_{31}(n, \varepsilon), \tau_{32}(n, \varepsilon)\right\}, \\
G_{3}(n, \varepsilon)=\left(\varepsilon^{-1} c_{n}\right)^{-1 / 2} \tilde{\Psi}^{-1 / 2}\left(\alpha_{3}(n, \varepsilon), \varepsilon^{-1} c_{n}\right) \cdot G\left(\nu_{n}(\varepsilon), \tau_{\min }(n, \varepsilon)\right), \\
G(S, T)=\left(\begin{array}{ll}
\int_{S}^{T} \tilde{Z}_{1}(t) \dot{x}_{t} d t & \int_{S}^{T} \tilde{Z}_{1}(t) x_{t} d t \\
\int_{S}^{T} \dot{x}_{t} x_{t} d t & \int_{S}^{T} x_{t}^{2} d t
\end{array}\right), \\
\vartheta_{3}(n, \varepsilon)=G^{-1}\left(\nu_{n}(\varepsilon), \tau_{\min }(n, \varepsilon)\right) \cdot \Phi\left(\nu_{n}(\varepsilon), \tau_{\min }(n, \varepsilon)\right), \\
\Phi(S, T)=\left(\begin{array}{ll}
\int_{S}^{T} \tilde{Z}_{1}(t) d \dot{x}_{t}, & \left.\int_{S}^{T} x_{t} d x_{t}\right)^{\prime} .
\end{array}\right.
\end{gathered}
$$

We introduce the following notation: put

$$
\Delta_{3}=\left(1+v_{0}^{2}\right)^{1 / 2} \frac{1 \vee E_{\vartheta}\left(Z_{1}^{\star \star}(0)\right)^{2}}{\left|E_{\vartheta} Z_{1}^{\star}(0) Z_{1}^{\star \star}(0)\right|}
$$

and define

$$
\begin{aligned}
& r_{3}^{\prime}=\left(\left[E_{\vartheta}\left(Z_{1}^{\star \star}(0)\right)^{2}\right] \wedge 1\right)^{-1} c_{\left[2 \delta_{3}^{-1} \varrho_{1} \Delta_{3}^{q}-1\right]_{1} \vee 1}, \\
& r_{3}^{\prime \prime}=\left(\left[E_{\vartheta}\left(Z_{1}^{\star \star}(0)\right)^{2}\right] \wedge 1\right)^{-1} c_{\left[2 \delta_{3}^{-1} \varrho_{1} \Delta_{3}^{q}\right]_{1}+1} .
\end{aligned}
$$

Corollary 3.3 Let the parameter $\vartheta$ in (34) belongs to the set $\tilde{\Theta}_{3}$. Then for any $\varepsilon>0$ the sequential plan SEP3( $(\varepsilon)$ defined in (70) is closed. It has the following properties:

$1^{\circ}$. for any $\varepsilon>0$

$$
\sup _{\vartheta \in \tilde{\Theta}_{3}}\left\|\vartheta_{3}(\varepsilon)-\vartheta\right\|_{q}^{2} \leq \delta_{3} \varepsilon
$$

$2^{\circ}$. the following relations hold with $P_{\vartheta}$ - probability one:

$$
0<r_{3}^{\prime} \leq \lim _{\varepsilon \rightarrow 0} \varepsilon T_{3}(\varepsilon) \leq \varlimsup_{\varepsilon \rightarrow 0} \varepsilon T_{3}(\varepsilon) \leq r_{3}^{\prime \prime}<\infty
$$

$3^{\circ}$. the estimator $\vartheta_{3}(\varepsilon)$ is strongly consistent:

$$
\lim _{\varepsilon \rightarrow 0} \vartheta_{3}(\varepsilon)=\vartheta \quad P_{\vartheta}-\text { a.s. }
$$


Proof. The proof of Corollary 3.3, except of the second assertion, follows from Theorem 1 directly. Assertion $2^{\circ}$ can be verify similar to the second assertion of Corollaries 3.1 and 3.2. Indeed, from the definition of stopping times $\tau_{31}(n, \varepsilon), \tau_{32}(n, \varepsilon)$ and (14), (58), (61), (62), (63) and (69), we can find the $P_{\vartheta}-$ a.s. limits

$$
\lim _{n \vee \varepsilon} \frac{\tau_{31}(n, \varepsilon)}{\varepsilon^{-1} c_{n}}=\left[E_{\vartheta}\left(Z_{1}^{\star \star}(0)\right)^{2}\right]^{-1}
$$

and

$$
\lim _{n \vee \varepsilon}\left[\tau_{32}(n, \varepsilon)-\varepsilon^{-1} c_{n}\right]=\ln 2 v_{0} C_{02}^{-2}, \quad \lim _{n \vee \varepsilon} G_{3}^{-1}(n, \varepsilon)=\left[E_{\vartheta}\left(Z_{1}^{\star \star}(0)\right)^{2} \vee 1\right] \cdot \tilde{G}_{3}
$$

and, by the definition of $\beta_{3}(n, \varepsilon)$ and $\Delta_{3}$ :

$$
\lim _{n \vee \varepsilon} \beta_{3}(n, \varepsilon)=\Delta_{3}^{-1} \quad P_{\vartheta}-\text { a.s. }
$$

The second assertion of Corollary 3.3 follows from (71)-(73) and definition (70) of stopping times $T_{3}(\varepsilon)$ and $\sigma_{3}(\varepsilon)$.

Hence Corollary 3.3 is proved.

\subsection{General sequential estimation procedure of the second-order autoregressive process}

In this point we construct the sequential estimation procedure for the parameters $\vartheta_{0}$ and $\vartheta_{1}$ of the process (34) on the bases of estimators, presented in the points 3.1-3.3.

Denote $\tilde{j}=\arg \min _{j=1,3} T_{j}(\varepsilon)$. Because in general it is unknown to which region $\vartheta$ belongs to, we define the sequential plan $(\tilde{T}(\varepsilon), \tilde{\vartheta}(\varepsilon))$ of estimation $\vartheta \in \tilde{\Theta}$ on the bases of all constructed above estimators by the formulae

$$
\widetilde{\operatorname{SEP}}(\varepsilon)=(\tilde{T}(\varepsilon), \tilde{\vartheta}(\varepsilon)), \quad \tilde{T}(\varepsilon)=T_{\tilde{j}}(\varepsilon), \quad \tilde{\vartheta}(\varepsilon)=\vartheta_{\tilde{j}}(\varepsilon) .
$$

THEOREM 2. Assume that the underlying process $\left(x_{t}\right)$ satisfies the equation (34), and for the numbers $\delta_{1}, \delta_{2}, \delta_{3}$ in the definitions (64), (65) and (70) of sequential plans the condition $\sum_{j=1}^{3} \delta_{j}=1$ is fulfilled. Then for any $\varepsilon>0$ and every $\vartheta \in \tilde{\Theta}$ the sequential estimation plans $(\tilde{T}(\varepsilon), \tilde{\vartheta}(\varepsilon))$ of $\vartheta$ are closed $\left(\tilde{T}(\varepsilon)<\infty \quad P_{\vartheta}-\right.$ a.s. $)$. They possess the following properties:

$1^{\circ}$. for any $\varepsilon>0$

$$
\sup _{\vartheta \in \tilde{\Theta}}\|\tilde{\vartheta}(\varepsilon)-\vartheta\|_{q}^{2} \leq \varepsilon
$$

$2^{\circ}$. the following relations hold with $P_{\vartheta}$ - probability one:

i) for $\vartheta \in \tilde{\Theta}_{1}$ :

- for $\vartheta \in \tilde{\Theta}_{11}$ (stationary case)

$$
\varlimsup_{\varepsilon \rightarrow 0} \varepsilon \tilde{T}(\varepsilon) \leq r_{11}^{\prime \prime}<\infty ;
$$


- for $\vartheta \in \tilde{\Theta}_{12}$ (purely explosive "complex" case)

$$
\varlimsup_{\varepsilon \rightarrow 0}\left[\tilde{T}(\varepsilon)-\frac{1}{2 v_{0}} \ln \varepsilon^{-1}\right] \leq r_{12}^{\prime \prime}<\infty ;
$$

- for $\vartheta \in \tilde{\Theta}_{13}:$

$$
\varlimsup_{\varepsilon \rightarrow 0} \sqrt{\varepsilon} \tilde{T}(\varepsilon) \leq r_{13}^{\prime \prime}<\infty,
$$

- for $\vartheta \in \tilde{\Theta}_{14}$ (purely explosive "real" case) the following relations hold with $P_{\vartheta}$ - probability one:

$$
\varlimsup_{\varepsilon \rightarrow 0}\left[\tilde{T}(\varepsilon)+\frac{1}{v_{0}} \ln \tilde{T}(\varepsilon)-\frac{1}{2 v_{0}} \ln \varepsilon^{-1}-\frac{1}{2 v_{0}} \ln c_{\sigma_{14}(\varepsilon)}\right] \leq r_{14}^{\prime \prime}<\infty ;
$$

ii) for $\vartheta \in \tilde{\Theta}_{2}$ (purely explosive "real" case) the following relations hold with $P_{\vartheta}$ - probability one:

$$
\varlimsup_{\varepsilon \rightarrow 0}\left[\tilde{T}(\varepsilon)-\frac{1}{2 v_{1}} \ln \varepsilon^{-1}\right] \leq r_{2}^{\prime \prime}<\infty ;
$$

iii) for $\vartheta \in \tilde{\Theta}_{3}$ (mixed case):

$$
\varlimsup_{\varepsilon \rightarrow 0} \varepsilon \tilde{T}(\varepsilon) \leq r_{3}^{\prime \prime}<\infty ;
$$

$3^{\circ}$. for $\vartheta \in \tilde{\Theta}$ the estimator $\tilde{\vartheta}(\varepsilon)$ is strongly consistent:

$$
\lim _{\varepsilon \rightarrow 0} \tilde{\vartheta}(\varepsilon)=\vartheta \quad P_{\vartheta}-\text { a.s. }
$$

Proof. The closeness of sequential plans $\widetilde{S E P}(\varepsilon)$ and assertions $2^{\circ}$ and $3^{\circ}$ of Theorem 2 follow from Corollaries 3.1-3.3 directly.

Now we prove the first assertion. To this end we show first, that all the stopping times $\tau_{1}(n, \varepsilon), \tau_{2}(n, \varepsilon), \tau_{31}(n, \varepsilon)$ and $\tau_{32}(n, \varepsilon)$ are $P_{\vartheta}$ - a.s.-finite for every $\vartheta \in \tilde{\Theta}$. The $P_{\vartheta}-$ a.s.-finiteness of stopping times $\tau_{1}(n, \varepsilon), \tau_{32}(n, \varepsilon)$ for every $\vartheta \in \tilde{\Theta}$ and $\tau_{2}(n, \varepsilon), \tau_{31}(n, \varepsilon)$ for $\vartheta \in \tilde{\Theta}_{2} \cup \tilde{\Theta}_{3}$ can be verified by making use of the definitions of these stopping times and the properties of the process under observation.

Then we will verify the finiteness of stopping times $\tau_{2}(n, \varepsilon)$ and $\tau_{31}(n, \varepsilon)$ for $\vartheta \in \tilde{\Theta}_{1}$. According to definitions (65) and (70) of these stopping times it is enough to show the divergence of the following integral

$$
\int_{0}^{\infty} \tilde{Z}_{1}^{2}(t) d t=\infty \quad P_{\vartheta}-\text { a.s. }
$$

where $\tilde{Z}_{1}(t)=X_{1}(t)-\hat{v}_{0}(t) X_{2}(t)$.

In the subsets of the set $\vartheta \in \tilde{\Theta}_{1}$ the estimator $\hat{v}_{0}(t)$, defined in (48) has the following $P_{\vartheta}-$ a.s. limits:

- for $\vartheta \in \tilde{\Theta}_{1 j}$

$$
\lim _{t \rightarrow \infty} \hat{v}_{0}(t)=\tilde{v}_{1 j}, \tilde{v}_{1 j}=\ln \frac{<\mathrm{e}^{A} F_{1 j}>_{22}}{<F_{1 j}>_{22}}, j=1,3,4 ;
$$


- for $\vartheta \in \tilde{\Theta}_{12}$

$$
\lim _{t \rightarrow \infty}\left|\hat{v}_{0}(t)-\tilde{v}_{12}(t)\right|=0, \quad \tilde{v}_{12}(t)=\ln \frac{<\mathrm{e}^{A} F_{12}(t)>_{22}}{<F_{12}(t)>_{22}} .
$$

Define $z_{11}(t)=X_{1}(t)-\tilde{v}_{11} X_{2}(t), Z_{11}(t)=\left(z_{11}(t), x_{t}\right)^{\prime}$ and

$$
\tilde{V}_{11}=\left(\begin{array}{ll}
1 & -\tilde{v}_{11} \\
0 & 1
\end{array}\right)
$$

From (36) it follows that $P_{\vartheta}-$ a.s.

$$
\lim _{T \rightarrow \infty} \frac{1}{T} \int_{0}^{T} Z_{11}(t) Z_{11}^{\prime}(t) d t=\tilde{V}_{11} F_{11} \tilde{V}_{11}^{\prime}
$$

and

$$
\lim _{T \rightarrow \infty} \frac{1}{T} \int_{0}^{T} z_{11}^{2}(t) d t>0 \quad P_{\vartheta}-\text { a.s. }
$$

Then by the definition of $(\tilde{Z}(t))$,

$$
\begin{aligned}
\lim _{T \rightarrow \infty} & \frac{1}{T} \int_{0}^{T} \tilde{Z}_{1}^{2}(t) d t=\lim _{T \rightarrow \infty}\left[\frac{1}{T} \int_{0}^{T} z_{11}^{2}(t) d t-\frac{2}{T} \int_{0}^{T}\left(\hat{v}_{0}(t)-\tilde{v}_{11}\right) z_{11}(t) x_{t} d t+\right. \\
+ & \left.\frac{1}{T} \int_{0}^{T}\left(\hat{v}_{0}(t)-\tilde{v}_{11}\right)^{2} x_{t}^{2} d t\right]=\lim _{T \rightarrow \infty} \frac{1}{T} \int_{0}^{T} z_{11}^{2}(t) d t>0 \quad P_{\vartheta}-\text { a.s. }
\end{aligned}
$$

and the relation (74) for the case $\tilde{\Theta}_{11}$ holds true.

The relation (74) can be verified in a similar way in the case $\tilde{\Theta}_{13} \cup \tilde{\Theta}_{14}$. In the case $\tilde{\Theta}_{12}$ the relation (74) is obviously.

Now we can prove the property $1^{\circ}$ of the estimator $\tilde{\vartheta}(\varepsilon)$. Define the constants $\rho_{2}=\rho_{1}, \rho_{3}=2 \rho_{1}$. By the definition of stopping times $\sigma_{j}(\varepsilon), j=\overline{1,3}$, similarly to the proof of Theorem 1 we get

$$
\begin{gathered}
\|\tilde{\vartheta}(\varepsilon)-\vartheta\|_{q}^{2} \leq\left(\sum_{n \geq 1} E_{\vartheta} \rho_{\tilde{j}}^{-1} \delta_{\tilde{j}} \beta_{\tilde{j}}^{q}(n, \varepsilon) \cdot\left\|\vartheta_{\tilde{j}}(n, \varepsilon)-\vartheta\right\|^{q}\right)^{2 / q} \leq \\
\leq\left(\sum_{j=1}^{3} \rho_{j}^{-1} \delta_{j} \cdot \sum_{n \geq 1} E_{\vartheta} \beta_{j}^{q}(n, \varepsilon)\left\|\vartheta_{j}(n, \varepsilon)-\vartheta\right\|^{q}\right)^{2 / q} .
\end{gathered}
$$

Due to the obtained finiteness properties of all the stopping times in these sums all the mathematical expectations are well-defined and following to the line of the proof of the first assertion of Theorem 1, we can estimate finally

$$
\|\tilde{\vartheta}(\varepsilon)-\vartheta\|_{q}^{2} \leq\left(\sum_{j=1}^{3} \delta_{j}\right)^{2 / q} \varepsilon=\varepsilon .
$$

Hence Theorem 2 is proved. 


\section{Sequential parameter estimation of a time delayed process}

In this section the general estimation procedure, constructed in the point 2.2 will be applied to the parameter estimation problem of a time delayed process (4).

Define $p=1, x(t)=X(t), a_{0}(t)=X(t), a_{1}(t)=X(t-1)$. Then the equation (1) has the form (4):

$$
d X(t)=\vartheta_{0} X(t) d t+\vartheta_{1} X(t-1) d t+d W(t) .
$$

To define $\Theta=\Theta^{*}$ we introduce the following notation, see [4] for details.

Let $s=u(r)(r<1)$ and $s=w(r)\left(r \in R^{1}\right)$ be the functions given by the parametric representation $(r(\xi), s(\xi))$ in $R^{2}$ :

$$
r(\xi)=\xi \cot \xi, s(\xi)=-\xi / \sin \xi
$$

with $\xi \in(0, \pi)$ and $\xi \in(\pi, 2 \pi)$ respectively.

Now we define the parameter set $\Theta^{*}$ to be the plane $R^{2}$ without some lines.

It seems to be not possible to construct a simple sequential procedure which has nice properties under $P_{\vartheta}$ for all $\vartheta \in \Theta^{*}$. Thus we are going to divide $\Theta^{*}$ in some smaller regions where it is possible to do.

To do it, let us consider the set $\Lambda$ of all (real or complex) roots of the so-called characteristic equation corresponding to (75)

$$
\lambda-\vartheta_{0}-\vartheta_{1} \mathrm{e}^{-\lambda}=0
$$

and put $v_{0}=v_{0}(\vartheta)=\max \{\operatorname{Re} \lambda \mid \lambda \in \Lambda\}$,

$$
v_{1}=v_{1}(\vartheta)=\max \left\{\operatorname{Re} \lambda \mid \lambda \in \Lambda, \operatorname{Re} \lambda<v_{0}\right\} .
$$

It can be easily shown that $-\infty<v_{1}<v_{0}<\infty$. By $m(\lambda)$ we denote the multiplicity of the solution $\lambda \in \Lambda$. Note that $m(\lambda)=1$ for all $\lambda \in \Lambda$ beside of the cases where $\vartheta_{1}=-e^{\vartheta_{0}-1}$. Then we have $\lambda=\vartheta_{0}-1 \in \Lambda$ and $m\left(\vartheta_{0}-1\right)=2$.

Now we are able to divide $\Theta^{*}$ into some appropriate for our purposes regions. Note, that this decomposition is very related to the classification used in [4], where can be found a figure giving an imagination of these sets. They have decomposed the plane $R^{2}$ in sets which they denoted by N, P1, P2, M1-M3, Q1-Q5. Here we use another notation, the Gushchin and Küchler notation is added for convenience.

DEFINITION $\left(\Theta^{*}\right)$. The parameter set $\Theta^{*}$ will be divided as

$$
\Theta^{*}=\Theta_{1}^{*} \cup \Theta_{2}^{*} \cup \Theta_{3}^{*}
$$

where

$$
\Theta_{1}^{*}=\Theta_{11}^{*} \cup \Theta_{12}^{*} \cup \Theta_{13}^{*}, \quad \Theta_{2}^{*}=\Theta_{21}^{*} \cup \Theta_{22}^{*}
$$

with

$\Theta_{11}^{*}=\left\{\vartheta \in R^{2} \mid v_{0}(\vartheta)<0\right\}$

$\Theta_{12}^{*}=\left\{\vartheta \in R^{2} \mid v_{0}(\vartheta)>0\right.$ and $\left.v_{0}(\vartheta) \notin \Lambda\right\}$, 
$\Theta_{13}^{*}=\left\{\vartheta \in R^{2} \mid v_{0}(\vartheta)>0 ; v_{0}(\vartheta) \in \Lambda, \quad m\left(v_{0}\right)=2\right\}$,

$\Theta_{21}^{*}=\left\{\vartheta \in R^{2} \mid v_{0}(\vartheta)>0, v_{0}(\vartheta) \in \Lambda, m\left(v_{0}\right)=1, v_{1}(\vartheta)>0\right.$ and $\left.v_{1}(\vartheta) \in \Lambda\right\},(M 2)$

$\Theta_{22}^{*}=\left\{\vartheta \in R^{2} \mid v_{0}(\vartheta)>0, v_{0}(\vartheta) \in \Lambda, m\left(v_{0}\right)=1, v_{1}(\vartheta)>0\right.$ and $\left.v_{1}(\vartheta) \notin \Lambda\right\}, \quad(P 1)$

$\Theta_{3}^{*}=\left\{\vartheta \in R^{2} \mid v_{0}(\vartheta)>0, \quad v_{0}(\vartheta) \in \Lambda, m\left(v_{0}\right)=1 \quad\right.$ and $\left.v_{1}(\vartheta)<0\right\}$,

and introduce, in addition,

$\Theta_{41}^{*}=\left\{\vartheta \in R^{2} \mid v_{0}(\vartheta)=0, v_{0}(\vartheta) \in \Lambda, m\left(v_{0}\right)=1\right\}$,

$\Theta_{42}^{*}=\left\{\vartheta \in R^{2} \mid v_{0}(\vartheta)=0, v_{0}(\vartheta) \in \Lambda, \quad m\left(v_{0}\right)=2\right\}$,

$\Theta_{43}^{*}=\left\{\vartheta \in R^{2} \mid v_{0}(\vartheta)>0, v_{0}(\vartheta) \in \Lambda, m\left(v_{0}\right)=1, v_{1}(\vartheta)=0\right.$ and $\left.v_{1}(\vartheta) \in \Lambda\right\}$,

The parameter set $\Theta^{*}$ equals the plane $R^{2}$ without the bounds of the set $\Theta_{12}^{*} \cup$ $\Theta_{13}^{*} \cup \Theta_{3}^{*}$. In particular, $\Theta_{11}^{*}$ is the set of parameters $\vartheta$ for which there exists a stationary solution of (75).

Obviously, by all sets $\Theta_{11}^{*}, \Theta_{12}^{*}, \Theta_{13}^{*}, \Theta_{21}^{*}, \Theta_{22}^{*}, \Theta_{3}^{*}$ are pairwise disjoint, the closure of $\Theta^{*}$ is the whole $R^{2}$ and the exceptional set $R^{2} \backslash \Theta^{*}$ has Lebesgue measure zero.

We shall consider the sequential estimation problem for the one-parametric set $\Theta_{4}^{*}=\Theta_{41}^{*} \cup \Theta_{42}^{*} \cup \Theta_{43}^{*}$ as well. This case is of interest in view of that the set $\Theta_{4}^{*}$ is the bound of the following regions: $\Theta_{11}^{*}, \Theta_{12}^{*}, \Theta_{21}^{*}, \Theta_{3}^{*}$. In this case $\vartheta_{1}=-\vartheta_{0}$ and (75) can be written as a differential equation of the first order. We do not consider the scalar case $\Theta_{4}^{*}$ as an example of the general estimation procedure because our method is intend for two- or more-parametric models. Moreover, for similar oneparametric model a sequential estimation procedure is constructed and investigated in [16], [18]. We shall use this procedure in point 4.5 with applications to the case $\Theta_{4}^{*}$.

It is well known, that the LSE, which equals here to the maximum likelihood estimator is of the form

$$
\hat{\vartheta}(T)=G^{-1}(T) \Phi(T),
$$

where $G(T)=\int_{0}^{T} \phi(t) \phi^{\prime}(t) d t$

$$
\phi(t)=\left(\begin{array}{c}
X(t) \\
X(t-1)
\end{array}\right), \Phi(T)=\int_{0}^{T} \phi(t) d X(t),
$$

has the optimal rate of convergence and is optimal in an asymptotic minimax sense for the cases $\vartheta \in \Theta_{11}^{*} \cup \Theta_{3}^{*}$, see [4].

If $T \rightarrow \infty$, then the smallest and the largest eigenvalues of the information matrix $G(T)$ tend to infinity but the rates of increase depend on $\vartheta$. Using [4] and [9]-[12] one can show that these eigenvalues have the following rates of increase (in the a.s. sense) for unboundedly increasing $T$ in the following considered regions:

Table 4

\begin{tabular}{|l|l|l|}
\hline Region & $\lambda_{\min }(G(T))$ & $\lambda_{\max }(G(T))$ \\
\hline$\Theta_{11}^{*}$ & $T$ & $T$ \\
\hline$\Theta_{12}^{*}$ & $e^{2 v_{0} T}$ & $e^{2 v_{0} T}$ \\
\hline$\Theta_{13}^{*}$ & $T^{-2} e^{2 v_{0} T}$ & $T^{2} e^{2 v_{0} T}$ \\
\hline$\Theta_{2}^{*}$ & $e^{2 v_{1} T}$ & $e^{2 v_{0} T}$ \\
\hline$\Theta_{3}^{*}$ & $T$ & $e^{2 v_{0} T}$ \\
\hline
\end{tabular}


Now we will use this knowledge for the investigation of the asymptotic properties of the weighted LSE. To this aim we introduce the weight matrices $V$ and $V(t)$ to obtain the transformed design matrix with equal rates of increase of its eigenvalues.

Let $\lambda=\mathrm{e}^{v_{0}}, Y(t)=X(t)-\lambda X(t-1)$ and put $V=I,(I-2 \times 2$ identity matrix $)$ in the case $\Theta_{1}^{*}$ and

$$
V=\left(\begin{array}{ll}
1 & -\lambda \\
1 & 0
\end{array}\right)
$$

in the cases $\Theta_{2}^{*}, \Theta_{3}^{*}$.

The parameter $\lambda=\mathrm{e}^{v_{0}}$ is a priori unknown because $v_{0}=v_{0}(\vartheta)$ depends on $\vartheta$. Thus we cannot use the matrix $V$, defined in (76) as a weight matrix. Therefore we shall change the parameter $\lambda$ in definition (76) by its estimator

$$
\lambda_{t}=\frac{\int_{0}^{t} X(s) X(s-1) d s}{\int_{0}^{t} X^{2}(s-1) d s}
$$

and define the weight matrix $V(t)$ in the cases $\Theta_{2}^{*}$ and $\Theta_{3}^{*}$ as follows:

$$
V(t)=\left(\begin{array}{ll}
1 & -\lambda_{t} \\
1 & 0
\end{array}\right) .
$$

Now we define the process $Y_{t}=X(t)-\lambda_{t} X(t-1)$ as an estimator of $Y(t)$.

Let us verify Assumptions (V) and $(\mathrm{G})$ for the case $\Theta_{1}^{*}$.

In the case $\Theta_{1}^{*}$ the minimal and maximal eigenvalues of the information matrix of the process (75) have equal rates of increase only in the cases $\Theta_{11}^{*}$ and $\Theta_{12}^{*}$. Indeed, according to [9] we have with $P_{\vartheta}-$ a.s. probability one

- for $\vartheta \in \Theta_{11}^{*}$

$$
\lim _{T \rightarrow \infty} T^{-1} G(T)=F_{11}^{*}
$$

- for $\vartheta \in \Theta_{12}^{*}$

$$
\lim _{T \rightarrow \infty}\left|\mathrm{e}^{-2 v_{0} T} G(T)-F_{12}^{*}(T)\right|=0
$$

The matrix $F_{11}^{*}$ is non-degenerate and the matrices $F_{12}^{*}(T), T>0$ are positive definite, periodic with the period $\Delta=2 \pi / \xi_{0}, \quad \xi_{0} \in(0, \pi)$ and $\inf _{T \in[0, \Delta]}\left|F_{12}^{*}(T)\right|>$ $0, \sup _{T \in[0, \Delta]}\left\|F_{12}^{*}(T)\right\|<\infty$ (see [4], [9]).

Similar to [11] we can get in the case $\Theta_{13}^{*}$ the following asymptotic relations for the processes $X(t)$ and $Y(t)=X(t)-e^{v_{0}} X(t-1)$ :

$$
\begin{aligned}
& \lim _{t \rightarrow \infty} t^{-1} e^{-v_{0} t} X(t)=2 U_{0} \quad P_{\vartheta}-\text { a.s. }, \\
& \lim _{t \rightarrow \infty} e^{-v_{0} t} Y(t)=2 U_{0} \quad P_{\vartheta}-\text { a.s. }
\end{aligned}
$$

where

$$
U_{0}=X_{0}(0)+\vartheta_{1} \int_{-1}^{0} \mathrm{e}^{-v_{0}(s+1)} X_{0}(s) d s+\int_{0}^{\infty} \mathrm{e}^{-v_{0} s} d W(s)
$$


As follows, with $P_{\vartheta}$-probability one, we have

$$
\begin{gathered}
\lim _{T \rightarrow \infty} \frac{1}{\mathrm{e}^{2 v_{0} T}} \int_{0}^{T} Y^{2}(t) d t=\frac{2 U_{0}^{2}}{v_{0}}, \\
\lim _{T \rightarrow \infty}\left|\frac{1}{T \mathrm{e}^{2 v_{0} T}} \int_{0}^{T} Y(t) X(t-1) d t-\frac{2 \mathrm{e}^{-v_{0}} U_{0}^{2}}{v_{0}}\left[1-\frac{1}{2 v_{0} T}\right]\right|=0, \\
\lim _{T \rightarrow \infty}\left|\frac{1}{T^{2} \mathrm{e}^{2 v_{0} T}} \int_{0}^{T} X^{2}(t-1) d t-\frac{2 \mathrm{e}^{-2 v_{0}} U_{0}^{2}}{v_{0}}\left[1-\frac{1}{v_{0} T}+\frac{1}{2 v_{0}^{2} T^{2}}\right]\right|=0 .
\end{gathered}
$$

From (78)-(80) we can find the $P_{\vartheta}-$ a.s. limits:

$$
\begin{aligned}
& \lim _{T \rightarrow \infty} \frac{1}{T^{2} \mathrm{e}^{2 v_{0} T}} G(T)=F_{13}^{*}, \quad F_{13}^{*}=\frac{2 U_{0}^{2}}{v_{0}}\left(\begin{array}{ll}
1 & \mathrm{e}^{-v_{0}} \\
\mathrm{e}^{-v_{0}} & \mathrm{e}^{-2 v_{0}}
\end{array}\right), \\
& \lim _{T \rightarrow \infty} \mathrm{e}^{-4 v_{0} T} \operatorname{det}(G(T))=\left(\frac{U_{0}}{v_{0}}\right)^{4} \mathrm{e}^{-2 v_{0}} .
\end{aligned}
$$

Then Assumptions $(\mathrm{V})$ and $(\mathrm{G})$ are fulfilled, where the functions $\varphi_{0}(\cdot)$ and $\varphi_{1}(\cdot)$ have the form

$$
\varphi_{0}(T)=\varphi_{1}(T)= \begin{cases}T, & \Theta_{11}^{*}, \\ \mathrm{e}^{2 v_{0} T}, & \Theta_{12}^{*}, \\ T^{2} \mathrm{e}^{2 v_{0} T}, & \Theta_{13}^{*}\end{cases}
$$

and $\Psi_{1}(\alpha, x)=x, g(\varphi) \equiv 1$ in the cases $\Theta_{11}^{*} \cup \Theta_{12}^{*}$ and $g\left(\varphi_{0}(T)\right)=\ln ^{8} \varphi_{0}(T) \sim T^{8}$ if $\vartheta \in \Theta_{13}^{*}$.

Now we verify Assumptions $(\mathrm{V})$ and $(\mathrm{G})$ for the case $\Theta_{2}^{*}$.

Similar to [11] we can get the following asymptotic relations for the processes $X(t), Y(t)=X(t)-e^{v_{0}} X(t-1)$ :

- for $\vartheta \in \Theta_{2}^{*}$

$$
\lim _{t \rightarrow \infty}\left|e^{-v_{0} t} X(t)-C_{2}\right|=0 P_{\vartheta}-\text { a.s.; }
$$

- for $\vartheta \in \Theta_{21}^{*}$

$$
\lim _{t \rightarrow \infty}\left|e^{-v_{1} t} Y(t)-C_{21}\right|=0 P_{\vartheta}-\text { a.s.; }
$$

- for $\vartheta \in \Theta_{22}^{*}$

$$
\lim _{t \rightarrow \infty}\left|e^{-v_{1} t} Y(t)-C_{22}(t)\right|=0 P_{\vartheta}-\text { a.s. },
$$

where $C_{22}(t)$ is a periodic bounded function.

Define $\lambda_{t}$ the estimator of $\lambda=\mathrm{e}^{v_{0}}$ as

$$
\lambda_{t}=\frac{\int_{0}^{t} X(s) X(s-1) d s}{\int_{0}^{t} X^{2}(s-1) d s}
$$


and $Y_{t}=X(t)-\lambda_{t} X(t-1)$. Then we have, similarly to the case $\tilde{\Theta}_{2}$, with $P_{\vartheta}-$ a.s. probability one

- for $\vartheta \in \Theta_{2}^{*}$

$$
\lim _{T \rightarrow \infty} \mathrm{e}^{-2 v_{0} T} \int_{0}^{T} X^{2}(t) d t=\tilde{C}_{2},
$$

- for $\vartheta \in \Theta_{21}^{*}$

$$
\begin{gathered}
\lim _{T \rightarrow \infty} \mathrm{e}^{-2 v_{1} T} \int_{0}^{T} Y^{2}(t) d t=\frac{C_{21}}{2 v_{1}}, \\
\lim _{t \rightarrow \infty} \mathrm{e}^{-v_{1} t} Y_{t}=c_{21}, \quad \lim _{T \rightarrow \infty} \mathrm{e}^{-2 v_{1} T} \int_{0}^{T} Y_{t}^{2} d t=\tilde{c}_{21} ;
\end{gathered}
$$

- for $\vartheta \in \Theta_{22}^{*}$

$$
\begin{gathered}
\lim _{T \rightarrow \infty}\left|\mathrm{e}^{-2 v_{1} T} \int_{0}^{T} Y^{2}(t) d t-\tilde{C}_{22}(T)\right|=0, \\
\lim _{t \rightarrow \infty}\left|\mathrm{e}^{-v_{1} t} Y_{t}-c_{22}(t)\right|=0, \quad \lim _{T \rightarrow \infty}\left|\mathrm{e}^{-2 v_{1} T} \int_{0}^{T} Y_{t}^{2} d t-\tilde{c}_{22}(T)\right|=0 .
\end{gathered}
$$

where $\tilde{C}_{22}(T), c_{22}(t)$ and $\tilde{c}_{22}(T)$ are some periodic bounded function.

Put $\bar{\varphi}(T)=\operatorname{diag}\left\{\mathrm{e}^{2 v_{1} T}, \mathrm{e}^{2 v_{0} T}\right\}, \alpha=\left(v_{0}, v_{1}\right), 0<v_{1}<v_{0}$ and $\Psi_{1}(\alpha, x)=x^{\tilde{\alpha}}, \tilde{\alpha}=$ $v_{0} / v_{1}$.

By making use of the obtained limiting relations we can find the following $P_{\vartheta}-$ a.s. limits

- in the case $\vartheta \in \Theta_{21}^{*}:$

$$
\begin{gathered}
\lim _{T: S \rightarrow \infty} \bar{G}(S, T)=G_{21}, \\
\lim _{T: S \rightarrow \infty} \tilde{G}^{-1}(S, T)=\tilde{G}_{21} ;
\end{gathered}
$$

- in the case $\vartheta \in \Theta_{22}^{*}$ :

$$
\begin{gathered}
\lim _{T: S \rightarrow \infty}\left\|\bar{G}(S, T)-G_{22}(T)\right\|=0, \\
\lim _{T: S \rightarrow \infty}\left\|\tilde{G}^{-1}(S, T)-\tilde{G}_{22}(T)\right\|=0 .
\end{gathered}
$$


The matrices $G_{21}, \tilde{G}_{21}$ are constant and the matrix $G_{21}$ is non-degenerate; the matrices $G_{22}(T), \tilde{G}_{22}(T)$ are periodic and $G_{22}(T)$ is non-degenerate for all $T>$ $0 \quad P_{\vartheta}$ - a.s. and such that $\inf _{T>0}\left|\lambda_{\min } G_{22}(T)\right|>0, \inf _{T>0}\left\|\tilde{G}_{22}(T)\right\|>0, \sup _{T>0}\left\|\tilde{G}_{22}(T)\right\|<$ $\infty$. Then, in particular, Assumptions (V) and (G) are fulfilled with $\bar{g}(T) \equiv 1$.

The case $\Theta_{3}^{*}$ was yet not fully considered in our previous papers. Then consider this case in more detail.

According to [4], [9] for the process $X(t)$ we have

$$
\lim _{t \rightarrow \infty}\left|e^{-v_{0} t} X(t)-C_{3}\right|=0 \quad P_{\vartheta}-\text { a.s. }
$$

and the process $Y(t)=X(t)-\lambda X(t-1), \lambda=\mathrm{e}^{v_{0}}$ is stationary. Here $C_{3}$ is some constant defined in [4].

We now verify all the assumptions of Theorem 1 .

Introduce following notation:

$$
\begin{gathered}
y_{0}(s)=x_{0}(t-s)-\lambda x_{0}(t-s-1), \\
Z_{1}(t)=\int_{-\infty}^{t} y_{0}(s) d W(s), \quad Z_{2}(T)=\int_{-\infty}^{T} e^{-v_{0}(T-t)} Z_{1}(t) d t, \\
Z_{3}(T)=\int_{-\infty}^{T} e^{-v_{0}(T-t)} Z_{2}(t) d t, \quad Z_{4}(T)=Z_{2}(T)-Z_{3}(T) .
\end{gathered}
$$

It should be noted that in the considered case $\Theta_{3}^{*}$ the processes $Z_{i}(t), i=\overline{1,3}$ are stationary Gaussian processes, continuous in probability, having a spectral density and, as follows, ergodic (see [19]).

According to the properties of the processes $X(t)$ and $Y(t)$ in considered case we have the following limiting relations with $P_{\vartheta}$-probability one:

$$
\begin{gathered}
\lim _{T \rightarrow \infty} \frac{1}{e^{2 v_{0} T}} \int_{0}^{T} X^{2}(t) d t=\frac{C_{3}^{2}}{2 v_{0}}, \quad \lim _{T \rightarrow \infty} \frac{1}{e^{2 v_{0} T}} \int_{0}^{T} X(t) X(t-1) d t=\frac{C_{3}^{2} \mathrm{e}^{-v_{0}}}{2 v_{0}}, \\
\lim _{t \rightarrow \infty}\left|Y(t)-Z_{1}(t)\right|=0, \quad \lim _{T \rightarrow \infty} \frac{1}{T} \int_{0}^{T} Y^{2}(t) d t=E Z_{1}^{2}(0), \\
\lim _{T \rightarrow \infty}\left|\frac{1}{e^{v_{0} T}} \int_{0}^{T} Y(t) X(t) d t-C_{3} Z_{2}(T)\right|=0 \\
\lim _{T \rightarrow \infty}\left|\frac{1}{e^{v_{0} T}} \int_{0}^{T} Y(t) X(t-1) d t-C_{3} e^{-v_{0}} Z_{2}(T)\right|=0 .
\end{gathered}
$$

We can get the following asymptotic properties with $P_{\vartheta}$-probability one for the estimators $\lambda_{t}$, defined in (81) and for the process $Y_{t}=X(t)-\lambda_{t} X(t-1)$ :

$$
\lim _{t \rightarrow \infty}\left|e^{v_{0} t}\left(\lambda_{t}-\lambda\right)-C_{3}^{-1} e^{v_{0}} Z_{2}(t)\right|=0, \quad \lim _{t \rightarrow \infty}\left|\left(\lambda_{t}-\lambda\right) X(t-1)-Z_{2}(t)\right|=0,
$$




$$
\begin{gathered}
\lim _{T \rightarrow \infty}\left|\frac{1}{e^{v_{0} T}} \int_{0}^{T} Y_{t} X(t) d t-C_{3} Z_{4}(T)\right|=0, \quad \lim _{t \rightarrow \infty}\left|Y_{t}-\left(Z_{1}(t)-Z_{2}(t)\right)\right|=0, \\
\lim _{T \rightarrow \infty}\left|\frac{1}{T} \int_{0}^{T} Y_{t} Y(t) d t-\frac{1}{T} \int_{0}^{T} Z_{1}(t)\left[Z_{1}(t)-Z_{2}(t)\right] d t\right|=0
\end{gathered}
$$

and, as follows,

$$
\lim _{T \rightarrow \infty} \frac{1}{T} \int_{0}^{T} Y_{t} Y(t) d t=\sigma_{12}, \quad \lim _{T \rightarrow \infty} \frac{1}{T} \int_{0}^{T} Y_{t}^{2} d t=\sigma_{22},
$$

where $\sigma_{12}=E Z_{1}(0)\left[Z_{1}(0)-Z_{2}(0)\right]=\int_{-\infty}^{0}\left[y_{0}^{2}(t)-\mathrm{e}^{v_{0} t} \int_{-\infty}^{t} y_{0}^{2}(s) d s\right] d t, \sigma_{22}=E\left[Z_{1}(0)-\right.$ $\left.Z_{2}(0)\right]^{2}$.

Then, putting

$$
V_{t}=\left(\begin{array}{ll}
1 & -\lambda_{t} \\
1 & 0
\end{array}\right), \quad V=\left(\begin{array}{ll}
1 & -\lambda \\
1 & 0
\end{array}\right)
$$

and, using Proposition 3 from Appendix, we get the following relation

$$
\lim _{T \rightarrow \infty} \bar{G}(T)=G_{3} \quad P_{\vartheta}-\text { a.s. }
$$

$G_{3}=\operatorname{diag}\left\{\sigma_{12}, C_{3}^{2} / 2 v_{0}\right\}$ and Assumptions (V), $(\mathrm{G}),(\varphi \psi)$ and $(\Psi)$ hold true with $\varphi_{0}(T)=T, \varphi_{1}(T)=\mathrm{e}^{2 v_{0} T}, \bar{g}(T) \equiv 1, \Psi_{1}(\alpha, x)=\mathrm{e}^{2 \alpha x}, \alpha=v_{0}, \Psi\left[\alpha, \bar{i}_{2}\right]=\left(x, \mathrm{e}^{2 \alpha x}\right) \notin$ $P_{2}(\alpha), l_{0}=1, l_{1}=1, m=1$.

Thus all the Assumptions (V), (G), $(\varphi \Psi)$ and $(\Psi)$ for the set $\Theta^{*}$ are fulfilled by $l_{0}=2, m=0$ for $\vartheta \in \Theta_{1}^{*} \cup \Theta_{2}^{*}$ and $l_{0}=1, l_{1}=1, m=1$ for $\vartheta \in \Theta_{3}^{*}$ as well as if we put the set $A=(0,+\infty)$ in the cases $\Theta_{12}^{*} \cup \Theta_{13}^{*} \cup \Theta_{3}, A=\left\{\left(v_{0}, v_{1}\right): 0<v_{1}<v_{0}<\right.$ $\infty\}$ in the case $\Theta_{2}^{*}$ and

Table 5

\begin{tabular}{|c||l|l||l|l|}
\hline Region & $b_{0}(t)$ & $b_{1}(T)$ & $\bar{b}_{0}(t)$ & $\bar{b}_{1}(T)$ \\
\hline$\Theta_{11}^{*}$ & $X(t)$ & $X(t-1)$ & $X(t)$ & $X(t-1)$ \\
\hline$\Theta_{12}^{*}$ & $X(t)$ & $X(t-1)$ & $X(t)$ & $X(t-1)$ \\
\hline$\Theta_{13}^{*}$ & $Y_{t}$ & $X(t)$ & $Y(t)$ & $X(t)$ \\
\hline$\Theta_{2}^{*}$ & $Y_{t}$ & $X(t)$ & $Y(t)$ & $X(t)$ \\
\hline$\Theta_{3}^{*}$ & $Y_{t}$ & $X(t)$ & $Y(t)$ & $X(t)$ \\
\hline
\end{tabular}

Table 6

\begin{tabular}{|c||l|l||l|l|l|}
\hline Region & $\varphi_{0}(\alpha, T)$ & $\varphi_{1}(\alpha, T)$ & $\alpha$ & $\Psi_{1}(\alpha, x)$ & $g(\varphi)$ \\
\hline$\Theta_{11}^{*}$ & $T$ & $T$ & - & $x$ & 1 \\
\hline$\Theta_{12}^{*}$ & $e^{2 v_{0} T}$ & $e^{2 v_{0} T}$ & $v_{0}$ & $x$ & 1 \\
\hline$\Theta_{13}^{*}$ & $T^{2} e^{2 v_{0} T}$ & $T^{2} e^{2 v_{0} T}$ & $v_{0}$ & $x$ & $\ln ^{8} \varphi$ \\
\hline$\Theta_{2}^{*}$ & $e^{2 v_{1} T}$ & $e^{2 v_{0} T}$ & $\left(v_{0}, v_{1}\right)$ & $x^{v_{0} / v_{1}}$ & 1 \\
\hline$\Theta_{3}^{*}$ & $T$ & $e^{2 v_{0} T}$ & $v_{0}$ & $e^{2 v_{0} x}$ & 1 \\
\hline
\end{tabular}


Now we shall apply the general estimation procedure (20) to the cases $\Theta_{1}^{*}, \Theta_{2}^{*}, \Theta_{3}^{*}$ separately. Then we shall define, similar to the first example, the final sequential estimation procedure, which works in $\Theta^{*}$, using these estimators. In addition, we shall construct the estimation plans for the one-parametric case $\Theta_{4}^{*}$.

We shall give the proofs in more detail only in the cases $\Theta_{3}^{*}$ and $\Theta_{4}^{*}$ because all the necessary asymptotic properties of the observed process $(X(t))$ for other regions are given in our previous papers [9]-[12].

\subsection{Estimation procedure for the case $\Theta_{1}^{*}$}

In the definition of the general sequential estimation plan (20) we put $V(t)=I$ and $\nu_{n}(\varepsilon)=0, n \geq 1$ and in the definition of stopping times $\tau(n, \varepsilon)$ we take $\Psi_{1}\left(\alpha(n, \varepsilon), \varepsilon^{-1} c_{n}\right)=\varepsilon^{-1} c_{n}$.

Denote $\operatorname{SEP} 1(\varepsilon)=\left(T_{1}(\varepsilon), \vartheta_{1}(\varepsilon)\right)$ the sequential plan (20) with these parameters, which in considered cases has the form:

$$
T_{1}(\varepsilon)=\tau_{1}\left(\sigma_{1}(\varepsilon), \varepsilon\right), \quad \vartheta_{1}(\varepsilon)=S_{1}^{-1}\left(\sigma_{1}(\varepsilon), \varepsilon\right) \sum_{n=1}^{\sigma_{1}(\varepsilon)} \beta_{1}^{q}(n, \varepsilon) \vartheta_{1}(n, \varepsilon),
$$

where

$$
\begin{gathered}
\tau_{1}(n, \varepsilon)=\inf \left\{T>0:\left(\int_{0}^{T} X^{2}(t) d t\right)^{q / 2}+\left(\int_{0}^{T} X^{2}(t-1) d t\right)^{q / 2}=\left(\varepsilon^{-1} c_{n}\right)^{q / 2}\right\} \\
\sigma_{1}(\varepsilon)=\inf \left\{N \geq 1: S_{1}(N, \varepsilon) \geq \delta_{1}^{-1} \varrho_{1}\right\}
\end{gathered}
$$

$\delta_{1} \in(0,1)$ is some arbitrary chosen constant and

$$
\begin{gathered}
\varrho_{1}=b_{q} 2^{\frac{q-2}{q}} \sum_{n \geq 1} c_{n}^{-q / 2} ; \quad S_{1}(N, \varepsilon)=\sum_{n=1}^{N} \beta_{1}^{q}(n, \varepsilon), \quad \beta_{1}(n, \varepsilon)=\left\|G_{1}^{-1}(n, \varepsilon)\right\|^{-1}, \\
G_{1}(n, \varepsilon)=\left(\varepsilon^{-1} c_{n}\right)^{-1} G\left(\tau_{1}(n, \varepsilon)\right), \quad \Phi_{1}(n, \varepsilon)=\left(\varepsilon^{-1} c_{n}\right)^{-1} \Phi\left(\tau_{1}(n, \varepsilon)\right), \\
G(T)=\int_{0}^{T} \phi(t) \phi^{\prime}(t) d t, \quad \phi(t)=\left(\begin{array}{c}
X(t) \\
X(t-1)
\end{array}\right), \quad \Phi(T)=\int_{0}^{T} \phi(t) d X(t), \\
\vartheta_{1}(n, \varepsilon)=G_{1}^{-1}(n, \varepsilon) \cdot \Phi_{1}(n, \varepsilon) .
\end{gathered}
$$

It should be pointed out, that for $q=2$ the sequential plan SEP1 $(\varepsilon)$ coincides with the sequential plan, presented in [9].

Now we introduce the following notation:

$$
\begin{gathered}
\bar{f}_{11}=\left[<F_{11}^{*}>_{11}^{q / 2}+<F_{11}^{*}>_{22}^{q / 2}\right]^{2 / q}, \quad \bar{\Delta}_{11}=\bar{f}_{11} \cdot\left\|\left(F_{11}^{*}\right)^{-1}\right\|, \\
\bar{r}_{11}^{\prime}=\bar{f}_{11}^{-1} \cdot c_{\left[\delta_{1}^{-1} \bar{\Delta}_{11}^{q} \varrho_{1}-1\right]_{1} \vee 1}, \quad \bar{r}_{11}^{\prime \prime}=\bar{f}_{11}^{-1} \cdot c_{\left[\delta_{1}^{-1} \bar{\Delta}_{11}^{q} \varrho_{1}\right]_{1}+1} ; \\
\bar{f}_{12}^{\prime}=\left[\sup _{T>0}\left(<F_{12}^{*}(T)>_{11}^{q / 2}+<F_{12}^{*}(T)>_{22}^{q / 2}\right)\right]^{2 / q}
\end{gathered}
$$




$$
\begin{gathered}
\bar{f}_{12}^{\prime \prime}=\left[\inf _{T>0}\left(<F_{12}^{*}(T)>_{11}^{q / 2}+<F_{12}^{*}(T)>_{22}^{q / 2}\right)\right]^{2 / q}, \\
\bar{\Delta}_{12}^{\prime}=\bar{f}_{12}^{\prime \prime} \cdot \inf _{T>0}\left\|\left(F_{12}^{*}(T)\right)^{-1}\right\|, \quad \bar{\Delta}_{12}^{\prime \prime}=\bar{f}_{12}^{\prime} \cdot \sup _{T>0}\left\|\left(F_{12}^{*}(T)\right)^{-1}\right\|, \\
\bar{r}_{12}^{\prime}=\frac{1}{2 v_{0}} \ln \left(\bar{f}_{12}^{\prime}\right)^{-1} \cdot c_{\left[\delta_{1}^{-1} \rho_{1}\left(\bar{\Delta}_{12}^{\prime}\right)^{q}-1\right]_{1} \vee 1}, \quad \bar{r}_{12}^{\prime \prime}=\frac{1}{2 v_{0}} \ln \left(\bar{f}_{12}^{\prime \prime}\right)^{-1} \cdot c_{\left[\delta_{1}^{-1} \rho_{1}\left(\bar{\Delta}_{12}^{\prime \prime}\right)^{q}\right]_{1}+1} ; \\
\bar{f}_{13}=\left[<F_{13}^{*}>_{11}^{q / 2}+<F_{13}^{*}>_{22}^{q / 2}\right]^{2 / q}, \quad \bar{\Delta}_{13}=\bar{f}_{13} \mathrm{e}^{2 v_{0}}\left(v_{0} U_{0}^{-1}\right)^{4}\left\|F_{13}^{*}\right\|, \\
\sigma_{13}(\varepsilon)=\left[\delta_{1}^{-1} \varrho_{1} \bar{\Delta}_{13}^{q} \ln ^{4 q} \varepsilon^{-1}\right]_{1}+1, \bar{r}_{13}^{\prime}=\frac{1}{2 v_{0}} \ln \bar{f}_{13}^{-1} c_{\left[\delta_{1}^{-1} \rho_{1} \bar{\Delta}_{13}^{q}-1\right]_{1} \vee 1}, \bar{r}_{13}^{\prime \prime}=\frac{1}{2 v_{0}} \ln \bar{f}_{13} .
\end{gathered}
$$

The next corollary summarizes the basic properties of the constructed above estimators.

Corollary 4.1. Let the parameter $\vartheta$ in $(75)$ belongs to the set $\Theta_{1}^{*}$. Then for any $\varepsilon>0$ the sequential plan $\mathrm{SEP} 1(\varepsilon)$ defined in $(95)$ is closed. It has the following properties:

$1^{\circ}$. for any $\varepsilon>0$

$$
\sup _{\vartheta \in \Theta_{1}^{*}}\left\|\vartheta_{1}(\varepsilon)-\vartheta\right\|_{q}^{2} \leq \varepsilon \delta_{1}
$$

$2^{\circ}$. the following relations hold with $P_{\vartheta}$ - probability one:

- for $\vartheta \in \Theta_{11}^{*}$

$$
0<\bar{r}_{11}^{\prime} \leq \lim _{\varepsilon \rightarrow 0} \varepsilon T_{1}(\varepsilon) \leq \varlimsup_{\varepsilon \rightarrow 0} \varepsilon T_{1}(\varepsilon) \leq \bar{r}_{11}^{\prime \prime}<\infty,
$$

- for $\vartheta \in \Theta_{12}^{*}$

$$
0<\bar{r}_{12}^{\prime} \leq \varliminf_{\varepsilon \rightarrow 0}\left[T_{1}(\varepsilon)-\frac{1}{2 v_{0}} \ln \varepsilon^{-1}\right] \leq \varlimsup_{\varepsilon \rightarrow 0}\left[T_{1}(\varepsilon)-\frac{1}{2 v_{0}} \ln \varepsilon^{-1}\right] \leq \bar{r}_{12}^{\prime \prime}<\infty ;
$$

- for $\vartheta \in \Theta_{13}^{*}$

$$
\begin{gathered}
\frac{\lim _{\varepsilon \rightarrow 0}}{}\left[T_{1}(\varepsilon)+\frac{1}{v_{0}} \ln T_{1}(\varepsilon)-\frac{1}{2 v_{0}} \ln \varepsilon^{-1}\right] \geq \bar{r}_{13}^{\prime}>0, \\
\varlimsup_{\varepsilon \rightarrow 0}\left[T_{1}(\varepsilon)+\frac{1}{v_{0}} \ln T_{1}(\varepsilon)-\frac{1}{2 v_{0}} \ln \varepsilon^{-1}-\frac{1}{2 v_{0}} \ln c_{\sigma_{13}(\varepsilon)}\right] \leq \bar{r}_{13}^{\prime \prime}<\infty ;
\end{gathered}
$$

$3^{\circ}$. the estimator $\vartheta_{1}(\varepsilon)$ is strongly consistent:

$$
\lim _{\varepsilon \rightarrow 0} \vartheta_{1}(\varepsilon)=\vartheta \quad P_{\vartheta}-\text { a.s. }
$$

Proof. The proof of Corollary 4.1 is similar to the proof of Corollary 3.1.

Remark 4.1. Similar to Remark 3.2, the asymptotic constants $\bar{r}_{11}^{\prime}$ and $\bar{r}_{11}^{\prime \prime}$ in the stationary case $\Theta_{11}^{*}$ can be changed by $\bar{r}_{11}^{\prime}=\bar{r}_{11}^{\prime \prime}=\bar{f}_{11}^{-1}$ (it coincides with the optimal convergence rate of the MLE) by appropriate chosen sequences $\left(c_{n}\right)$ and $\left(\kappa_{n}\right)$ and in the case $\Theta_{13}^{*}$, for $c_{n}=o\left(\mathrm{e}^{\left(n^{a}\right)}\right)$ as $n \rightarrow \infty, a=1 / 4 q, \ln c_{\sigma_{13}(\varepsilon)}=o\left(\ln \varepsilon^{-1}\right)$, as $\varepsilon \rightarrow 0$ and

$$
\lim _{\varepsilon \rightarrow 0} \frac{T_{1}(\varepsilon)}{\ln \varepsilon^{-1}}=\frac{1}{2 v_{0}} \quad P_{\vartheta}-\text { a.s. }
$$




\subsection{Estimation procedure for the case $\Theta_{2}^{*}$}

We put in the definition of the general sequential estimation plan (20)

$$
V(t)=\left(\begin{array}{ll}
1 & -\lambda_{t} \\
1 & 0
\end{array}\right)
$$

where $\lambda_{t}$ is defined in (81) and

$$
\nu_{2}(n, \varepsilon)=\inf \left\{T>0: \int_{0}^{T} Y_{t}^{2} d t=\left(\varepsilon^{-1} c_{n}\right)^{\delta}\right\}
$$

$\delta \in(0,1)$ is some arbitrary chosen number.

In the definition of stopping times $\tau(n, \varepsilon)$ we take $\Psi_{1}\left(\alpha(n, \varepsilon), \varepsilon^{-1} c_{n}\right)=\left(\varepsilon^{-1} c_{n}\right)^{\tilde{\alpha}_{2}(n, \varepsilon)}$,

$$
\tilde{\alpha}_{2}(n, \varepsilon)=\frac{\ln \int_{0}^{\nu_{2}(n, \varepsilon)} X^{2}(t) d t}{\delta \ln \varepsilon^{-1} c_{n}} .
$$

Denote SEP2 $(\varepsilon)=\left(T_{2}(\varepsilon), \vartheta_{2}(\varepsilon)\right)$ sequential plan (20) with these parameters, which in considered cases has the form:

$$
T_{2}(\varepsilon)=\tau_{2}\left(\sigma_{2}(\varepsilon), \varepsilon\right), \quad \vartheta_{2}(\varepsilon)=S_{2}^{-1}\left(\sigma_{2}(\varepsilon), \varepsilon\right) \sum_{n=1}^{\sigma_{2}(\varepsilon)} \beta_{2}^{q}(n, \varepsilon) \vartheta_{2}(n, \varepsilon),
$$

where

$$
\begin{aligned}
\tau_{2}(n, \varepsilon) & =\inf \left\{T>\nu_{2}(n, \varepsilon):\left(\frac{1}{\varepsilon^{-1} c_{n}} \int_{\nu_{2}(n, \varepsilon)}^{T} Y_{t}^{2} d t\right)^{q / 2}+\right. \\
+ & \left.\left(\frac{1}{\left(\varepsilon^{-1} c_{n}\right)^{\tilde{\alpha}_{2}(n, \varepsilon)}} \int_{\nu_{2}(n, \varepsilon)}^{T} X^{2}(t) d t\right)^{q / 2}=1\right\}, \\
& \sigma_{2}(\varepsilon)=\inf \left\{N \geq 1: S_{2}(N, \varepsilon) \geq \delta_{2}^{-1} \varrho_{1}\right\},
\end{aligned}
$$

$\delta_{2} \in(0,1)$ is some arbitrary chosen constant;

$$
\begin{gathered}
S_{2}(N, \varepsilon)=\sum_{n=1}^{N} \beta_{2}^{q}(n, \varepsilon), \beta_{2}(n, \varepsilon)=\left\|G_{2}^{-1}(n, \varepsilon)\right\|^{-1}, \\
G_{2}(n, \varepsilon)=\left(\varepsilon^{-1} c_{n}\right)^{-\frac{1}{2}} \tilde{\Psi}^{-\frac{1}{2}}(n, \varepsilon) G\left(\nu_{2}(n, \varepsilon), \tau_{2}(n, \varepsilon)\right), \\
\Phi_{2}(n, \varepsilon)=\tilde{\Psi}^{-\frac{1}{2}}(n, \varepsilon) \Phi\left(\nu_{2}(n, \varepsilon), \tau_{2}(n, \varepsilon)\right),{ }^{\top} G(T)=\int_{0}^{T} \phi(t) \phi^{\prime}(t) d t, \\
\phi(t)=\left(\begin{array}{c}
Y_{t} \\
X(t)
\end{array}\right), \Phi(T)=\int_{0}^{T} \phi(t) d X(t),
\end{gathered}
$$




$$
\vartheta_{2}(n, \varepsilon)=G_{2}^{-1}(n, \varepsilon) \cdot \Phi_{2}(n, \varepsilon) .
$$

Now we introduce following notation:

$$
\begin{gathered}
\bar{\alpha}_{21}:=\frac{v_{1} \ln \tilde{C}_{2}-v_{0} \ln \tilde{c}_{21}}{2 v_{1}^{2}}, \quad \bar{\alpha}_{22}:=\sup _{T>0} \frac{v_{1} \ln \tilde{C}_{2}-v_{0} \ln \tilde{c}_{22}(T)}{2 v_{1}^{2}}, \\
\tilde{\alpha}_{22}:=\inf _{T>0} \frac{v_{1} \ln \tilde{C}_{2}-v_{0} \ln \tilde{c}_{22}(T)}{2 v_{1}^{2}}, \quad \tilde{C}_{21}^{\prime}=\sup _{T>0} \tilde{c}_{21}(T), \quad \tilde{C}_{21}^{\prime \prime}=\inf _{T>0} \tilde{c}_{21}(T)
\end{gathered}
$$

and let $\bar{s}_{21}$ is the positive root of the equation

$$
\tilde{C}_{2} \cdot \mathrm{e}^{\frac{\bar{\alpha}_{21} q}{2 \delta}} \cdot s^{\alpha}+\left(\tilde{c}_{21}\right)^{q / 2} \cdot s-1=0,
$$

$\bar{s}_{22}$ and $\tilde{s}_{22}$ are the positive roots of the following equations

$$
\tilde{C}_{2} \cdot \mathrm{e}^{\frac{\bar{\alpha}_{22} q}{2 \delta}} \cdot s^{\alpha}+\left(\tilde{C}_{21}^{\prime}\right)^{q / 2} \cdot s-1=0
$$

and

$$
\tilde{C}_{2} \cdot \mathrm{e}^{\frac{\tilde{\alpha}_{22 q} q}{2 \delta}} \cdot s^{\alpha}+\left(\tilde{C}_{21}^{\prime \prime}\right)^{q / 2} \cdot s-1=0
$$

respectively;

$$
\begin{gathered}
\bar{S}_{21}=\operatorname{diag}\left\{\frac{2 / q}{s_{21}}, \mathrm{e}^{\frac{-\bar{\alpha}_{21}}{2 \delta}} \bar{s}_{21}^{\frac{v_{0}+v_{1}}{q v_{1}}}\right\}, \quad \bar{S}_{22}=\operatorname{diag}\left\{\frac{2 / q}{s_{22}}, \mathrm{e}^{\frac{-\bar{\alpha}_{22}}{2 \delta}} \bar{s}_{22}^{\frac{v_{0}+v_{1}}{q v_{1}}}\right\}, \\
\tilde{S}_{22}=\operatorname{diag}\left\{\tilde{s}_{22}^{2 / q}, \mathrm{e}^{\frac{-\tilde{\alpha}_{22}}{2 \delta} \tilde{s}_{22} \frac{v_{0}+v_{1}}{q v_{1}}}\right\}, \quad \bar{\Delta}_{21}=\left\|\tilde{G}_{21} \cdot \bar{S}_{21}\right\|, \\
\bar{\Delta}_{22}=\inf _{T>0}\left\|\tilde{G}_{22}(T) \cdot \bar{S}_{22}\right\|, \quad \tilde{\Delta}_{22}=\sup _{T>0}\left\|\tilde{G}_{22}(T)\right\| \cdot\left\|\tilde{S}_{22}\right\|
\end{gathered}
$$

and define

$$
\begin{array}{ll}
\bar{r}_{21}^{\prime}=\frac{1}{2 v_{1}} \ln \bar{s}_{21}^{-2 / q} \cdot c_{\left[\delta_{2}^{-1} \varrho_{1} \bar{\Delta}_{21}^{q}-1\right]_{1} \vee 1}, & \bar{r}_{21}^{\prime \prime}=\frac{1}{2 v_{1}} \ln \bar{s}_{21}^{-2 / q} \cdot c_{\left[\delta_{2}^{-1} \varrho_{1} \bar{\Delta}_{21}^{q}\right]_{1}+1}, \\
\bar{r}_{22}^{\prime}=\frac{1}{2 v_{1}} \ln \tilde{s}_{22}^{-2 / q} \cdot c_{\left[\delta_{2}^{-1} \varrho_{1} \bar{\Delta}_{22}^{q}-1\right]_{1} \vee 1}, & \bar{r}_{22}^{\prime \prime}=\frac{1}{2 v_{1}} \ln \bar{s}_{22}^{-2 / q} \cdot c_{\left[\delta_{2}^{-1} \varrho_{1} \tilde{\Delta}_{22}^{q}\right]_{1}+1} .
\end{array}
$$

Corollary 4.2. Let the parameter $\vartheta$ in (75) belongs to the set $\Theta_{2}^{*}$. Then for any $\varepsilon>0$ the sequential plan $\mathrm{SEP} 2(\varepsilon)$ defined in $(97)$ is closed. It has the following properties:

$1^{\circ}$. for any $\varepsilon>0$

$$
\sup _{\vartheta \in \Theta_{2}^{*}}\left\|\vartheta_{2}(\varepsilon)-\vartheta\right\|_{q}^{2} \leq \delta_{2} \varepsilon
$$

$2^{\circ}$. the following relation holds with $P_{\vartheta}-$ probability one:

$$
0<\bar{r}_{2 i}^{\prime} \leq \varliminf_{\varepsilon \rightarrow 0}\left[T_{2}(\varepsilon)-\frac{1}{2 v_{1}} \ln \varepsilon^{-1}\right] \leq \varlimsup_{\varepsilon \rightarrow 0}\left[T_{2}(\varepsilon)-\frac{1}{2 v_{1}} \ln \varepsilon^{-1}\right] \leq \bar{r}_{2 i}^{\prime \prime}<\infty
$$

for $\vartheta \in \Theta_{2 i}^{*}, i=1,2$;

$3^{\circ}$. the estimator $\vartheta_{2}(\varepsilon)$ is strongly consistent:

$$
\lim _{\varepsilon \rightarrow 0} \vartheta_{2}(\varepsilon)=\vartheta \quad P_{\vartheta}-\text { a.s. }
$$


Proof. As we noted above, Assumptions (V), (G) $(\varphi \psi)$ and $(\psi)$ follow from equalities (82)-(87) and (89). Then, according to Theorem 1, for the proof of Corollary 2 it is sufficient to establish Assumption $(\alpha)$ and assertion $2^{\circ}$.

First, using the equalities (82), (84) and (86), by the definition (96) of the estimator $\tilde{\alpha}_{2}(n, \varepsilon)$, we find its $P_{\vartheta}$-a.s. convergence rate: as $n \rightarrow \infty$ or $\varepsilon \rightarrow 0$,

$$
\begin{gathered}
\nu_{2}(n, \varepsilon)\left(\tilde{\alpha}_{2}(n, \varepsilon)-\alpha\right)=\nu_{2}(n, \varepsilon)\left(\frac{\ln \int_{0}^{\nu_{2}(n, \varepsilon)} X^{2}(t) d t}{\ln \int_{0}^{\nu_{2}(n, \varepsilon)} Y_{t}^{2} d t}-\frac{v_{0}}{v_{1}}\right)= \\
=\nu_{2}(n, \varepsilon) \cdot \frac{v_{1} \ln \mathrm{e}^{-2 v_{0} \nu_{2}(n, \varepsilon)} \int_{0}^{\nu_{2}(n, \varepsilon)} X^{2}(t) d t-v_{0} \ln \mathrm{e}^{-2 v_{1} \nu_{2}(n, \varepsilon)} \int_{0}^{\nu_{2}(n, \varepsilon)} Y_{t}^{2} d t}{2 v_{1}^{2} \nu_{2}(n, \varepsilon)+v_{1} \ln \mathrm{e}^{-2 v_{1} \nu_{2}(n, \varepsilon)} \int_{0}^{\nu_{2}(n, \varepsilon)} Y_{t}^{2} d t} \rightarrow \bar{\alpha}_{12}
\end{gathered}
$$

in the case $\Theta_{21}^{*}$ and, analogously, in the case $\Theta_{21}^{*}$, as $n \rightarrow \infty$ or $\varepsilon \rightarrow 0$,

$$
\nu_{2}(n, \varepsilon)\left(\tilde{\alpha}_{2}(n, \varepsilon)-\alpha\right)-\frac{v_{1} \ln \tilde{C}_{2}-v_{0} \ln \tilde{c}_{22}\left(\nu_{2}(n, \varepsilon)\right)}{2 v_{1}^{2}} \rightarrow 0 \quad P_{\vartheta}-\text { a.s. }
$$

Thus

$$
\begin{gathered}
\lim _{n \vee \varepsilon} \ln \left(\varepsilon^{-1} c_{n}\right)^{\left(\alpha-\tilde{\alpha}_{2}(n, \varepsilon)\right) \frac{q}{2}}=\frac{q}{2} \lim _{n \vee \varepsilon} \nu_{2}(n, \varepsilon)\left(\alpha-\tilde{\alpha}_{2}(n, \varepsilon)\right) \cdot \\
\cdot \nu_{2}^{-1}(n, \varepsilon)(\varepsilon) \ln \varepsilon^{-1} c_{n}=\frac{\bar{\alpha}_{21} q}{2 \delta} \quad P_{\vartheta}-\text { a.s. }
\end{gathered}
$$

and, as follows, with $P_{\vartheta}$-probability one

$$
\lim _{n \vee \varepsilon}\left(\varepsilon^{-1} c_{n}\right)^{\left(\alpha-\tilde{\alpha}_{2}(n, \varepsilon)\right) \frac{q}{2}}=\mathrm{e}^{\frac{\bar{\alpha}_{21} q}{2 \delta}}
$$

in the case $\Theta_{21}^{*}$ and in the case $\Theta_{22}^{*}$

$$
0<\mathrm{e}^{\frac{\tilde{\alpha}_{22} q}{2 \delta}} \leq \lim _{n \vee \varepsilon}\left(\varepsilon^{-1} c_{n}\right)^{\left(\alpha-\tilde{\alpha}_{2}(n, \varepsilon)\right) \frac{q}{2}} \leq \lim _{n \vee \varepsilon}\left(\varepsilon^{-1} c_{n}\right)^{\left(\alpha-\tilde{\alpha}_{2}(n, \varepsilon)\right) \frac{q}{2}} \leq \mathrm{e}^{\frac{\bar{\alpha}_{22 q} q}{2 \delta}}<\infty .
$$

Assumptions $(\alpha)$ are proved. By the definition of stopping times $\tau_{2}(n, \varepsilon)$ for $\vartheta \in \Theta_{21}^{*}$ we have

$$
\begin{gathered}
\lim _{n \rightarrow \infty}\left[\left(\frac{1}{\varepsilon^{-1} c_{n}} \int_{\nu_{2}(n, \varepsilon)}^{\tau_{2}(n, \varepsilon)} Y_{t}^{2} d t\right)^{q / 2}+\left(\frac{1}{\left(\varepsilon^{-1} c_{n}\right)^{\tilde{\alpha}_{2}(n, \varepsilon)}} \int_{\nu_{2}(n, \varepsilon)}^{\tau_{2}(n, \varepsilon)} X^{2}(t) d t\right)^{q / 2}\right]= \\
=\lim _{n \rightarrow \infty}\left[\left(\frac{1}{\mathrm{e}^{2 v_{1} \tau_{2}(n, \varepsilon)}} \int_{0}^{\tau_{2}(n, \varepsilon)} Y_{t}^{2} d t\right)^{q / 2} \cdot\left(\frac{\mathrm{e}^{2 v_{1} \tau_{2}(n, \varepsilon)}}{\varepsilon^{-1} c_{n}}\right)^{q / 2}+\right. \\
\left.+\left(\frac{1}{\mathrm{e}^{2 v_{0} \tau_{2}(n, \varepsilon)}} \int_{0}^{\tau_{2}(n, \varepsilon)} X^{2}(t) d t\right)^{q / 2} \cdot\left(\varepsilon^{-1} c_{n}\right)^{\left(\alpha-\tilde{\alpha}_{2}(n, \varepsilon)\right) \frac{q}{2}} \cdot\left(\frac{\mathrm{e}^{2 v_{1} \tau_{2}(n, \varepsilon)}}{\varepsilon^{-1} c_{n}}\right)^{q \alpha / 2}\right]=
\end{gathered}
$$




$$
=\lim _{n \rightarrow \infty}\left[\tilde{c}_{21}^{q / 2} \cdot\left(\frac{\mathrm{e}^{2 v_{1} \tau_{2}(n, \varepsilon)}}{\varepsilon^{-1} c_{n}}\right)^{q / 2}+\tilde{C}_{2}^{q / 2} \cdot \mathrm{e}^{\frac{\bar{\alpha}_{21} q}{2 \delta}} \cdot\left(\frac{\mathrm{e}^{2 v_{1} \tau_{2}(n, \varepsilon)}}{\varepsilon^{-1} c_{n}}\right)^{q \alpha / 2}\right]=1 .
$$

Then

$$
\lim _{n \vee \varepsilon} \mathrm{e}^{-2 v_{1} \tau_{2}(n, \varepsilon)} \varepsilon^{-1} c_{n}=\frac{2 / q}{s_{21}} \quad P_{\vartheta}-\text { a.s. }
$$

and, as follows, taking into account (88), (90) and (98), (99), $P_{\vartheta}-$ a.s.

$$
\begin{aligned}
& \lim _{n \vee \varepsilon} G_{2}^{-1}(n, \varepsilon)=\tilde{G}_{21} \cdot \bar{S}_{21}, \\
& \lim _{n \vee \varepsilon} \beta_{2}(n, \varepsilon)=\bar{\Delta}_{21}^{-1}
\end{aligned}
$$

in the case $\Theta_{21}^{*}$ and

$$
\begin{gathered}
\frac{2 / q}{s_{22}} \leq \lim _{n \vee \varepsilon} \mathrm{e}^{-2 v_{1} \tau_{2}(n, \varepsilon)} \varepsilon^{-1} c_{n} \leq \varlimsup_{n \vee \varepsilon} \mathrm{e}^{-2 v_{1} \tau_{2}(n, \varepsilon)} \varepsilon^{-1} c_{n} \leq \tilde{s}_{22}^{2 / q}, \\
\tilde{\Delta}_{22}^{-1} \leq \varliminf_{n \vee \varepsilon} \beta_{2}(n, \varepsilon) \leq \varlimsup_{n \vee \varepsilon} \beta_{2}(n, \varepsilon) \leq \bar{\Delta}_{22}^{-1}
\end{gathered}
$$

in the case $\Theta_{22}^{*}$. From the definition (97) and (100)-(103) follows the second assertion of Corollary 4.2.

Hence Corollary 4.2 is proved.

\subsection{Estimation procedure for the case $\Theta_{3}^{*}$}

Chose the non-random functions $\nu_{3}(n, \varepsilon), n \geq 1, \varepsilon>0$, satisfying the following conditions as $\varepsilon \rightarrow 0$ or $n \rightarrow \infty$ :

$$
\nu_{3}(n, \varepsilon)=o\left(\varepsilon^{-1} c_{n}\right), \quad \frac{\log ^{1 / 2} \nu_{3}(n, \varepsilon)}{\mathrm{e}^{v_{0} \nu_{3}(n, \varepsilon)}} \varepsilon^{-1} c_{n}=o(1) .
$$

Example: $\nu_{3}(n, \varepsilon)=\log ^{2} \varepsilon^{-1} c_{n}$.

Note, that for the functions, satisfying (104) the conditions (14)-(16) hold true.

Put $\alpha(n, \varepsilon):=\alpha_{3}(n, \varepsilon)=\ln \lambda_{\nu_{3}(n, \varepsilon)}$, where $\lambda_{t}$ is defined in (81). Now we verify, in the $P_{\vartheta}-$ a.s. sense Assumptions $(\alpha)$ using Proposition 3 from the Appendix:

$$
\begin{aligned}
& \lim _{n \vee \varepsilon} \ln \frac{\tilde{\Psi}_{22}(n, \varepsilon)}{\Psi_{22}(\alpha, n, \varepsilon)}=\lim _{n \vee \varepsilon} 2\left(\alpha_{3}(n, \varepsilon)-\alpha\right) \varepsilon^{-1} c_{n}=\lim _{n \vee \varepsilon} 2 \lambda^{-1}\left(\lambda_{\nu_{3}(n, \varepsilon)}-\lambda\right) \varepsilon^{-1} c_{n}= \\
= & 2 C_{3}^{-1} \lim _{n \vee \varepsilon} \frac{Z_{2}\left(\nu_{3}(n, \varepsilon)\right) \varepsilon^{-1} c_{n}}{\mathrm{e}^{v_{0} \nu_{3}(n, \varepsilon)}}=2 C_{3}^{-1} \lim _{n \vee \varepsilon} \frac{Z_{2}\left(\nu_{3}(n, \varepsilon)\right)}{\log ^{1 / 2} \nu_{3}(n, \varepsilon)} \cdot \frac{\log ^{1 / 2} \nu_{3}(n, \varepsilon)}{\mathrm{e}^{v_{0} \nu_{3}(n, \varepsilon)}} \cdot \varepsilon^{-1} c_{n}=0,
\end{aligned}
$$

then

$$
\lim _{n \vee \varepsilon} \frac{\tilde{\Psi}_{i i}(n, \varepsilon)}{\Psi_{i i}(\alpha, n, \varepsilon)}=1, i=1,2 \quad P_{\vartheta}-\text { a.s. }
$$


and all the conditions of Theorem 1 hold true.

Denote SEP3 $(\varepsilon)=\left(T_{3}(\varepsilon), \vartheta_{3}(\varepsilon)\right)$ the sequential plan $(20)$ with these parameters, which in considered case has the form:

$$
T_{3}(\varepsilon)=\tau_{\max }\left(\sigma_{3}(\varepsilon), \varepsilon\right), \quad \vartheta_{3}(\varepsilon)=S_{3}^{-1}\left(\sigma_{3}(\varepsilon), \varepsilon\right) \sum_{n=1}^{\sigma_{3}(\varepsilon)} \beta_{3}^{q}(n, \varepsilon) \vartheta_{3}(n, \varepsilon),
$$

where

$$
\begin{gathered}
\tau_{\max }\left(\sigma_{3}(\varepsilon), \varepsilon\right)=\max \left\{\tau_{31}\left(\sigma_{3}(\varepsilon), \varepsilon\right), \tau_{32}\left(\sigma_{3}(\varepsilon), \varepsilon\right)\right\}, \\
\tau_{31}(n, \varepsilon)=\inf \left\{T>0: \int_{\nu_{3}(n, \varepsilon)}^{T} Y_{t}^{2} d t=\varepsilon^{-1} c_{n}\right\}, \\
\tau_{32}(n, \varepsilon)=\inf \left\{T>0: \int_{\nu_{3}(n, \varepsilon)}^{T} X^{2}(t) d t=\mathrm{e}^{2 \alpha_{3}(n, \varepsilon) \varepsilon^{-1} c_{n}}\right\}, \\
\sigma_{3}(\varepsilon)=\inf \left\{N \geq 1: S_{3}(N, \varepsilon) \geq 2 \delta_{3}^{-1} \varrho_{1}\right\},
\end{gathered}
$$

$\delta_{3} \in(0,1)$ is some arbitrary chosen constant;

$$
\begin{aligned}
& S_{3}(N, \varepsilon)=\sum_{n=1}^{N} \beta_{3}^{q}(n, \varepsilon), \quad \beta_{3}(n, \varepsilon)=\left\|G_{3}^{-1}(n, \varepsilon)\right\|^{-1}, \\
& \tau_{\min }(n, \varepsilon)=\min \left\{\tau_{31}(n, \varepsilon), \tau_{32}(n, \varepsilon)\right\}, \\
& G_{3}(n, \varepsilon)=\left(\varepsilon^{-1} c_{n}\right)^{-1 / 2} \tilde{\Psi}^{-1 / 2}(n, \varepsilon) G\left(\nu_{3}(n, \varepsilon), \tau_{\min }(n, \varepsilon)\right) \text {, } \\
& \tilde{\Psi}(n, \varepsilon)=\left(\varepsilon^{-1} c_{n}, \mathrm{e}^{2 \alpha_{3}(n, \varepsilon) \varepsilon^{-1} c_{n}}\right), \quad G(T)=\int_{0}^{T} \phi(t) \phi^{\prime}(t) d t, \\
& \phi(t)=\left(\begin{array}{c}
Y_{t} \\
X(t)
\end{array}\right), \Phi(T)=\int_{0}^{T} \phi(t) d X(t), \\
& \vartheta_{3}(n, \varepsilon)=G^{-1}\left(\nu_{3}(n, \varepsilon), \tau_{\min }(n, \varepsilon)\right) \Phi\left(\nu_{3}(n, \varepsilon), \tau_{\min }(n, \varepsilon)\right) .
\end{aligned}
$$

Now we introduce following notation:

$$
g_{31}=\sigma_{12}^{-1}\left[\sigma_{22} \vee 1\right], \quad g_{32}=\sigma_{12}\left[\sigma_{22}^{-1} \wedge 1\right] \mathrm{e}^{-v_{0}}, \quad \bar{\Delta}_{3}=\sqrt{g_{31}^{2}+g_{32}^{2}}
$$

and define

$$
\bar{r}_{3}^{\prime}=\left[\sigma_{22}^{-1} \vee 1\right] \cdot c_{\left[2 \delta_{3}^{-1} \varrho_{1} \bar{\Delta}_{3}^{q}-1\right]_{1} \vee 1,} \quad \bar{r}_{3}^{\prime \prime}=\left[\sigma_{22}^{-1} \vee 1\right] \cdot c_{\left[2 \delta_{3}^{-1} \varrho_{1} \bar{\Delta}_{3}^{q}\right]_{1}+1}
$$

Corollary 4.3. Let the parameter $\vartheta$ in (75) belongs to the set $\Theta_{3}^{*}$. Then for any $\varepsilon>0$ the sequential plan SEP3 $(\varepsilon)$ defined in (105) is closed. It has the following properties:

$1^{\circ}$. for any $\varepsilon>0$

$$
\sup _{\vartheta \in \Theta_{3}^{*}}\left\|\vartheta_{3}(\varepsilon)-\vartheta\right\|_{q}^{2} \leq \varepsilon \delta_{3}
$$


$2^{\circ}$. the following relations hold with $P_{\vartheta}$ - probability one:

$$
0<\bar{r}_{3}^{\prime} \leq \lim _{\varepsilon \rightarrow 0} \varepsilon T_{3}(\varepsilon) \leq \varlimsup_{\varepsilon \rightarrow 0} \varepsilon T_{3}(\varepsilon) \leq \bar{r}_{3}^{\prime \prime}<\infty ;
$$

$3^{\circ}$. the estimator $\vartheta_{3}(\varepsilon)$ is strongly consistent:

$$
\lim _{\varepsilon \rightarrow 0} \vartheta_{3}(\varepsilon)=\vartheta \quad P_{\vartheta}-\text { a.s. }
$$

Proof. The proof of Corollary 4.3, except of second assertion, follows from Theorem 1 directly. Assertion $2^{\circ}$ can be verified similarly to the second assertion of Corollary 3.3. Indeed, from the definition of stopping times $\tau_{31}(n, \varepsilon), \tau_{32}(n, \varepsilon)$ and (14), (92), (93) we can find the limits with $P_{\vartheta}$-probability one:

$$
\lim _{n \vee \varepsilon} \varepsilon \tau_{31}(n, \varepsilon)=\sigma_{22}^{-1} c_{n}
$$

and

$$
\begin{gathered}
\lim _{n \vee \varepsilon}\left[\tau_{32}(n, \varepsilon)-\varepsilon^{-1} c_{n}\right]=\frac{1}{2 v_{0}} \ln 2 v_{0} C_{3}^{-2}, \\
\lim _{n \vee \varepsilon} \varepsilon \tau_{\min }(n, \varepsilon)=\left[\sigma_{22}^{-1} \wedge 1\right] c_{n}, \\
\lim _{n \vee \varepsilon} \varepsilon \tau_{\max }(n, \varepsilon)=\left[\sigma_{22}^{-1} \vee 1\right] c_{n}
\end{gathered}
$$

and, according to (92)-(104), (106)

$$
\lim _{n \vee \varepsilon} G_{3}^{-1}(n, \varepsilon)=\left(\begin{array}{ll}
g_{31} & 0 \\
-g_{32} & 0
\end{array}\right)
$$

and, by the definition of $\beta_{3}(n, \varepsilon)$ and $\Delta_{3}$,

$$
\lim _{n \vee \varepsilon} \beta_{3}(n, \varepsilon)=\bar{\Delta}_{3}^{-1}
$$

The second assertion of Corollary 5.3 follows from (107), (108) and definition (105).

Hence Corollary 4.3 is proved.

\subsection{General sequential estimation procedure for the set $\Theta^{*}$ of the special time-delayed process}

In this point we construct the sequential estimation procedure for the parameters $\vartheta_{0}$ and $\vartheta_{1}$ of the process (75) from the set $\Theta^{*}$ on the bases of estimators, presented in points 4.1-4.3.

Denote $j^{\star}=\arg \min _{j=1,3} T_{j}(\varepsilon)$. We define the sequential plan $\left(T^{\star}(\varepsilon), \vartheta^{\star}(\varepsilon)\right)$ of estimation $\vartheta \in \Theta^{*}$ on the bases of all constructed above estimators by the formulae $\operatorname{SEP}^{\star}(\varepsilon)=\left(T^{\star}(\varepsilon), \vartheta^{\star}(\varepsilon)\right)$,

$$
T^{\star}(\varepsilon)=T_{j^{\star}}(\varepsilon), \quad \vartheta^{*}(\varepsilon)=\vartheta_{j^{\star}}(\varepsilon) .
$$


THEOREM 3. Assume that the underlying process $(X(t))$ satisfies the equation (75), and for the numbers $\delta_{1}, \delta_{2}, \delta_{3}$ in the definitions (95), (97), (105) of sequential plans the condition $\sum_{j=1}^{3} \delta_{j}=1$ is fulfilled. Then for any $\varepsilon>0$ and every $\vartheta \in \Theta^{*}$ the sequential estimation plans $\left(T^{\star}(\varepsilon), \vartheta^{\star}(\varepsilon)\right)$ of $\vartheta$ are closed $\left(T^{\star}(\varepsilon)<\infty \quad P_{\vartheta}-\right.$ a.s. $)$. They possess the following properties:

$1^{\circ}$. for any $\varepsilon>0$

$$
\sup _{\vartheta \in \Theta^{*}}\left\|\vartheta^{\star}(\varepsilon)-\vartheta\right\|_{q}^{2} \leq \varepsilon ;
$$

$2^{\circ}$. the following relations hold with $P_{\vartheta}$ - probability one:

i) for $\vartheta \in \Theta_{1}$ :

- for $\vartheta \in \Theta_{11}^{*}$

$$
\varlimsup_{\varepsilon \rightarrow 0} \varepsilon T^{\star}(\varepsilon) \leq \bar{r}_{11}^{\prime \prime}<\infty
$$

- for $\vartheta \in \Theta_{12}^{*}$

$$
\varlimsup_{\varepsilon \rightarrow 0}\left[T^{\star}(\varepsilon)-\frac{1}{2 v_{0}} \ln \varepsilon^{-1}\right] \leq \bar{r}_{12}^{\prime \prime}<\infty ;
$$

- for $\vartheta \in \Theta_{13}^{*}$

$$
\varlimsup_{\varepsilon \rightarrow 0}\left[T^{\star}(\varepsilon)+\frac{1}{v_{0}} \ln T^{\star}(\varepsilon)-\frac{1}{2 v_{0}} \ln \varepsilon^{-1}-\frac{1}{2 v_{0}} \ln c_{\sigma_{13}(\varepsilon)}\right] \leq \bar{r}_{13}^{\prime \prime}<\infty ;
$$

ii) for $\vartheta \in \Theta^{*}{ }_{2 i}$ :

$$
\varlimsup_{\varepsilon \rightarrow 0}\left[T^{\star}(\varepsilon)-\frac{1}{2 v_{1}} \ln \varepsilon^{-1}\right] \leq \bar{r}_{2 i}^{\prime \prime}<\infty, \quad i=1,2 ;
$$

iii) for $\vartheta \in \Theta_{3}^{*}$ :

$$
\varlimsup_{\varepsilon \rightarrow 0} \varepsilon T^{\star}(\varepsilon) \leq \bar{r}_{3}^{\prime \prime}<\infty ;
$$

$3^{\circ}$. for $\vartheta \in \Theta^{*}$ the estimator $\vartheta^{\star}(\varepsilon)$ is strongly consistent:

$$
\lim _{\varepsilon \rightarrow 0} \vartheta^{\star}(\varepsilon)=\vartheta \quad P_{\vartheta}-\text { a.s. }
$$

Proof. The closeness of sequential plans $\operatorname{SEP}^{\star}(\varepsilon)$ and assertions $2^{\circ}$ and $3^{\circ}$ of Theorem 3 follow from Corollaries 4.1-4.3 directly. The proof of the first assertion is similar to Theorem 2 if we taking into account that the integrals

$$
\begin{aligned}
& \int_{0}^{\infty} X^{2}(t) d t=\infty \quad P_{\vartheta}-\text { a.s. } \\
& \int_{0}^{\infty}[X(t)-X(t-1)]^{2} d t=\infty \quad P_{\vartheta}-\text { a.s. }
\end{aligned}
$$

in all the cases $\Theta_{1}^{*}, \Theta_{2}^{*}, \Theta_{3}^{*}$ and, as follows, all the stopping times $\tau_{1}(n, \varepsilon), \tau_{2}(n, \varepsilon), \tau_{31}(n, \varepsilon)$ and $\tau_{32}(n, \varepsilon)$ are $P_{\vartheta}-$ a.s.-finite for every $\varepsilon>0$ and all $n \geq 1$.

The properties (109) can be established by using the asymptotic properties of the process $(\mathrm{X}(\mathrm{t})$ ) (see proofs of Corollaries 4.1-4.3 and [4], [9]-[12]).

Hence Theorem 3 is proved. 


\subsection{Estimation procedure for the case $\Theta_{4}^{*}$}

The set $\Theta_{4}^{*}$ is the bound of the following regions: $\Theta_{11}^{*}, \Theta_{12}^{*}, \Theta_{21}^{*}, \Theta_{3}^{*}$. In this case $\vartheta_{1}=-\vartheta_{0}$ and $(75)$ can be written as the differential equation of the first order:

$$
d X(t)=\vartheta_{0} a_{t} d t+d W(t), t \geq 0,
$$

where $a_{t}=X(t)-X(t-1)$.

We shall use sequential estimation plan SEP4 $(\varepsilon)=\left(T_{4}(\varepsilon), \vartheta_{4}^{*}(\varepsilon)\right)$ of the parameter $\vartheta=\vartheta_{0}(1,-1)^{\prime}$ with the $\varepsilon$-accuracy in the sense of the $L_{q}$-norm, which has similar structure to considered for Case II in [9] and has the form:

$$
\begin{gathered}
T_{4}(\varepsilon)=\inf \left\{T>0: \int_{0}^{T} a_{t}^{2} d t=2 b_{q}^{2 / q} \varepsilon^{-1}\right\}, \quad \vartheta_{4}^{*}(\varepsilon)=\vartheta_{04}^{*}(\varepsilon)(1,-1)^{\prime}, \\
\vartheta_{04}^{*}(\varepsilon)=\varepsilon\left(2 b_{q}^{2 / q}\right)^{-1} \cdot \int_{0}^{T_{4}(\varepsilon)} a_{t} d X(t) .
\end{gathered}
$$

Denote $h_{0}(T)=\theta^{-1}(T) T^{2}$, where $\theta(T), T \geq 0$ is any positive unboundedly increasing function, $h_{1}(T)=T^{2} \ln ^{2} T$, and

$$
\begin{gathered}
\left.A=\frac{1-\mathrm{e}^{-v_{0}}}{v_{0}-\vartheta_{0}+1} \cdot\left(X_{0}(0)\right)-\vartheta_{0} \int_{-1}^{0} \mathrm{e}^{-v_{0}(s+1)} X_{0}(s) d s+\int_{0}^{\infty} \mathrm{e}^{-v_{0} s} d W(s)\right), \\
\bar{A}=-\frac{1}{2 v_{0}} E_{\vartheta} A^{2}, \quad C_{43}=\frac{1}{2 v_{0}} \ln \left[2 b_{q}^{2 / q} \bar{A}^{-1}\right] .
\end{gathered}
$$

Corollary 4.5. Let in (75) the parameter $\vartheta \in \Theta_{4}^{*}$. Then for any $\varepsilon>0$ the sequential plan $\mathrm{SEP} 4(\varepsilon)$ defined in (110) is closed. It has the following properties:

$1^{\circ}$.

$$
\sup _{\vartheta \in \Theta_{4}^{*}}\left\|\vartheta_{4}^{*}(\varepsilon)-\vartheta\right\|_{q}^{2} \leq \varepsilon
$$

$2^{\circ}$. the following relations hold with $P_{\vartheta}-$ probability one:

- for $\vartheta \in \Theta_{41}^{*}$ :

$$
\lim _{\varepsilon \rightarrow 0} \varepsilon \cdot T_{4}(\varepsilon)=2 b_{q}^{2 / q} f_{a}^{-1},
$$

where $f_{a}$ is defined in (111) below;

- for $\vartheta \in \Theta_{42}^{*}$ :

$$
2^{-1} b_{q}^{2 / q} \leq \lim _{\varepsilon \rightarrow 0} \varepsilon \cdot h_{1}\left(T_{4}(\varepsilon)\right), \quad \varlimsup_{\varepsilon \rightarrow 0} \varepsilon \cdot h_{0}\left(T_{4}(\varepsilon)\right)=0 ;
$$

- for $\vartheta \in \Theta_{43}^{*}:$

$$
\lim _{\varepsilon \rightarrow 0}\left[T_{4}(\varepsilon)-\frac{1}{2 v_{0}} \ln \varepsilon^{-1}\right]=C_{43} ;
$$

$3^{\circ}$. the estimator $\vartheta_{4}^{*}(\varepsilon)$ is strongly consistent:

$$
\lim _{\varepsilon \rightarrow 0} \vartheta_{4}^{*}(\varepsilon)=\vartheta \quad P_{\vartheta}-\text { a.s. }
$$


Proof. The proof of the first assertion of Corollary 4.5 follows from [16]. For the proof of assertion $2^{\circ}$ we find the rates of increase for the integral $\int_{0}^{T} a_{t}^{2} d t$ as $T \rightarrow \infty$ in all the considered cases $\Theta_{41}^{*}, \Theta_{42}^{*}, \Theta_{43}^{*}$. According to [4] the process $a(t)$ satisfies the equality

$$
\begin{gathered}
a(t)=y_{0}(t) X_{0}(t)+\vartheta_{1} \int_{-1}^{0} y_{0}(t-s-1) X_{0}(s) d s+\int_{0}^{t} y_{0}(t-s) d W(s), t \geq 0, \\
y_{0}(t)=x_{0}(t)-x_{0}(t-1)
\end{gathered}
$$

and we have:

- for $\vartheta \in \Theta_{41}^{*}$ :

$y_{0}(t)=o\left(\mathrm{e}^{\gamma t}\right), \gamma<0$, as $t \rightarrow \infty$ and, as follows, there exists the positive constant limit

$$
\lim _{T \rightarrow \infty} \frac{1}{T} \int_{0}^{T} a_{t}^{2} d t=f_{a} \quad P_{\vartheta}-\text { a.s. }
$$

- for $\vartheta \in \Theta_{42}^{*}$ :

$y_{0}(t)=2+o\left(\mathrm{e}^{\gamma t}\right), \gamma<0$, as $t \rightarrow \infty$ and, as follows, $a_{t}=2 W(t)(1+o(1))$ as $t \rightarrow \infty$ and $P_{\vartheta}-$ a.s.

$$
\lim _{T \rightarrow \infty} \frac{1}{h_{0}(T)} \int_{0}^{T} a_{t}^{2} d t=\infty, \quad \varlimsup_{T \rightarrow \infty} \frac{1}{h_{1}(T)} \int_{0}^{T} a_{t}^{2} d t \leq 1 ;
$$

- for $\vartheta \in \Theta_{43}^{*}$ :

$y_{0}(t)=\frac{1-\mathrm{e}^{-v_{0}}}{v_{0}-\vartheta_{0}+1} \mathrm{e}^{v_{0} t}+o\left(\mathrm{e}^{\gamma t}\right)$, as $t \rightarrow \infty, \gamma<0$ and, as follows, $\mathrm{e}^{-v_{0} t} a_{t}=$ $A(1+o(1)) \quad P_{\vartheta}-$ a.s. and

$$
\lim _{T \rightarrow \infty} \frac{1}{\mathrm{e}^{2 v_{0} T}} \int_{0}^{T} a_{t}^{2} d t=\bar{A} \quad P_{\vartheta}-\text { a.s. }
$$

The second assertion of Corollary 4.5 follows from the definition (110) of the stopping time $T_{4}(\varepsilon)$ and (111)-(113).

The third assertion of Corollary 4.5 follows from the definition of sequential estimator $\vartheta_{4}^{*}(\varepsilon)$ and strong consistency of the LSE

$$
\hat{\vartheta}(T)=\frac{\int_{0}^{T} a_{t} d X(t)}{\int_{0}^{T} a_{t}^{2} d t}
$$

in the case $\Theta_{4}^{*}$ :

$$
\hat{\vartheta}(T)-\vartheta=\frac{\int_{0}^{T} a_{t} d W(t)}{\int_{0}^{T} a_{t}^{2} d t} \rightarrow 0 \quad P_{\vartheta}-\text { a.s. }
$$

Hence Corollary 4.5 is complete. 


\section{$5 \quad$ Appendix}

Proposition 1. Suppose, that Assumption (V) and (G) are fulfilled. Then the inequality (10) holds true. The inequality (11) is fulfilled under the additional condition (12).

Proof of Proposition 1. Define the matrix functions

$$
A(T)=V^{\prime} \bar{\varphi}^{-\frac{1}{2}}(T) \varphi_{0}^{\frac{1}{2}}(T), B(S, T)=\bar{\varphi}^{-\frac{1}{2}}(T) G(S) \tilde{\varphi}^{-\frac{1}{2}}(T) .
$$

Note, that $\bar{G}(S, T)=\bar{G}(T)-B(S, T)$. Taking into account that according to Assumption (V) the matrix $\bar{G}(T)$ is norm bounded from above $P_{\vartheta}-$ a.s. and due to the condition $\|A(T)\| \leq\|V\|$ for $T$ large enough, we obtain under Assumption $(\mathrm{G})$ :

$$
\begin{gathered}
\overline{\lim }_{T: S \uparrow \infty} \bar{g}^{-1}(T)\left\|\tilde{G}^{-1}(S, T)\right\|^{2}=\overline{\lim }_{T: S \uparrow \infty} \bar{g}^{-1}(T)\left\|A(T) \bar{G}^{-1}(S, T)\right\|^{2} \leq \\
\leq\|V\|^{2} \overline{\lim }_{T: S \uparrow \infty} \bar{g}^{-1}(T)\left\|\bar{G}^{-1}(S, T)\right\|^{2} \leq\|V\|^{2} \overline{\lim }_{T: S \uparrow \infty} \bar{g}^{-1}(T)\left\|\bar{G}^{-1}(T)\right\|^{2} . \\
\cdot\left\|\left(I-\bar{G}^{-1}(T) B(S, T)\right)^{-1}\right\|^{2} \quad P_{\vartheta}-\text { a.s. }
\end{gathered}
$$

Now we estimate the $P_{\vartheta}-$ a.s.-upper limit

$$
\begin{gathered}
\varlimsup_{T \rightarrow \infty} \bar{g}^{-1}(T)\left\|\bar{G}^{-1}(T)\right\|^{2}=\varlimsup_{T \rightarrow \infty} \bar{g}^{-1}(T) \operatorname{tr}\left[\bar{G}^{-1}(T)\left(\bar{G}^{\prime}(T)\right)^{-1}\right]= \\
=\varlimsup_{T \rightarrow \infty} \bar{g}^{-1}(T) \operatorname{tr}\left[\left(\bar{G}^{\prime}(T) \bar{G}(T)\right)^{-1}\right] \leq(p+1) \varlimsup_{T \rightarrow \infty} \bar{g}^{-1}(T) \lambda_{\max }\left[\left(\bar{G}^{\prime}(T) \bar{G}(T)\right)^{-1}\right]= \\
=(p+1)\left(\lim _{T \rightarrow \infty} \bar{g}(T) \lambda_{\min }\left[\bar{G}^{\prime}(T) \bar{G}(T)\right]\right)^{-1}<\infty .
\end{gathered}
$$

From the definition of the class $\mathcal{G}_{1}$ by Assumption (G) it follows, that as $T \rightarrow \infty$ and by $S=o(T)$, the following asymptotic relations $\varphi_{i}(S)=o\left(\bar{g}^{-1 / 2}(T) \varphi_{i}(T)\right)$ for $i=\overline{0, p}$ hold true.

Then

$$
\lim _{T: S \uparrow \infty} \bar{g}^{1 / 2}(T) \bar{\varphi}^{-1}(T) \cdot \bar{\varphi}(S)=0
$$

and

$$
\begin{gathered}
\lim _{T: S \uparrow \infty} \bar{g}^{1 / 2}(T) B(S, T)=\lim _{T: S \uparrow \infty}\left(\bar{g}^{1 / 2}(T) \bar{\varphi}^{-1}(T) \bar{\varphi}(S)\right)^{\frac{1}{2}} \cdot \bar{G}(S) . \\
\cdot\left(\bar{\varphi}(S) \bar{\varphi}^{-1}(T) \bar{g}^{1 / 2}(T)\right)^{\frac{1}{2}}=0 \quad P_{\vartheta}-\text { a.s. }
\end{gathered}
$$

As follows, $P_{\vartheta}-$ a.s.,

$$
\begin{gathered}
\varlimsup_{T: S \uparrow \infty}\|\bar{G}(S, T)\|=\varlimsup_{T \rightarrow \infty}\|\bar{G}(T)\|<\infty, \\
\lim _{T: S \uparrow \infty}\left\|\bar{G}^{-1}(T) B(S, T)\right\|=0
\end{gathered}
$$


and we obtain, finally, the inequality (10):

$$
\overline{\lim }_{T: S \uparrow \infty} \bar{g}^{-1}(T)\left\|\tilde{G}^{-1}(S, T)\right\|^{2}<\infty .
$$

The lower limiting bound for the norm $\left\|\tilde{G}^{-1}(S, T)\right\|^{2}$ can be obtained under the additional condition (12) and by making use of the following inequality from Lemma 2 of [14]:

$$
\lambda_{\max }\left\{A C A^{\prime}\right\} \geq \lambda_{\max }\left\{A A^{\prime}\right\} \cdot \lambda_{\min }\{C\},
$$

which holds true for any symmetric non-negative definite matrix $C$ and quadratic matrix $A$. Thus, for $S<T$ we have

$$
\begin{gathered}
\left.\| \tilde{G}^{-1}(S, T)\right) \|^{2}=\operatorname{tr}\left[\left(\left(A^{-1}(T)\right)^{\prime} \bar{G}^{\prime}(S, T) \bar{G}(S, T) A^{-1}(T)\right)^{-1}\right] \geq \\
\geq \lambda_{\max }\left\{\left(\left(A^{-1}(T)\right)^{\prime} \bar{G}^{\prime}(S, T) \bar{G}(S, T) A^{-1}(T)\right)^{-1}\right\}= \\
=\lambda_{\max }\left\{A(T)\left(\bar{G}^{\prime}(S, T) \bar{G}(S, T)\right)^{-1} A^{\prime}(T)\right\} \geq \\
\geq \lambda_{\max }\left\{A(T) A^{\prime}(T)\right\} \cdot \lambda_{\min }\left\{\left(\bar{G}^{\prime}(S, T) \bar{G}(S, T)\right)^{-1}\right\}=\lambda_{\max }\left\{V^{\prime}\left(\bar{\varphi}^{-1}(T) \varphi_{0}(T)\right) V\right\} . \\
\left.\cdot \lambda_{\max }^{-1}\left\{\bar{G}^{\prime}(S, T) \bar{G}(S, T)\right\} \geq \lambda_{\max }\left\{V^{\prime}\left(\bar{\varphi}^{-1}(T) \varphi_{0}(T)\right) V\right\} \cdot \| \bar{G}(S, T)\right) \|^{-2}
\end{gathered}
$$

and, as follows, the inequality (11) holds true

$$
\left.\lim _{T: S \uparrow \infty} \| \tilde{G}^{-1}(S, T)\right) \|^{2}>0 \quad P_{\vartheta}-\text { a.s. }
$$

Hence Proposition 1 holds.

Denote for every positive magnitude $h$ the difference $\Delta_{h} Z(t)=Z(t+h)-Z(t)$.

Proposition 2. Let $(Z(t))_{t \geq 0}$ be stationary Gaussian process with zero mean and such that for any $0<h \leq 1$ and every $t \in R^{1}$

$$
E\left(\Delta_{h} Z(t)\right)^{2} \leq C h .
$$

Then, as $t \rightarrow \infty$,

$$
Z(t)=O\left((\log t)^{\frac{1}{2}}\right) \quad P_{\vartheta}-\text { a.s. }
$$

Proof of Proposition 2. According to Theorem 2 in [15], p. 142, we have for all $t>0$ the inequalities

$$
P\left\{\sup _{[t, t+1]}|Z(s)|>\left(C_{1} \log t\right)^{1 / 2}\right\} \leq \exp \left\{-C_{1} C_{2} \log t\right\}=t^{-C_{1} C_{2}} .
$$

Here $C_{1}$ and $C_{2}$ are some positive constants.

Thus by the Borel-Cantelli lemma

$$
Z(t)=O\left((\log t)^{1 / 2}\right) \text { as } t \rightarrow \infty \quad P_{\vartheta}-\text { a.s. }
$$

Hence Proposition 2 holds. 
Proposition 3. Let the parameter $\vartheta$ of the process (75) belongs to the set $\Theta_{3}^{*}$. Then the processes $\left(Z_{i}(T)\right), i=\overline{1,4}$, defined in (91) have the following properties

$$
Z_{i}(t)=O\left((\log t)^{\frac{1}{2}}\right), i=\overline{1,4} \text { as } t \rightarrow \infty P_{\vartheta}-\text { a.s. }
$$

Proof of Proposition 3. First we show, that for any $0<h \leq 1$ and every $t \in R^{1}$ we have the inequalities:

$$
A(h)=E\left(\Delta_{h} Z_{1}(t)\right)^{2} \leq C h .
$$

Direct calculation gives the representation

$$
A(h)=\int_{t-h}^{t} y_{0}^{2}(t-s) d s+\int_{-\infty}^{t}\left(\Delta_{h} y_{0}(t-s)\right)^{2} d s .
$$

The function $y_{0}(\cdot)$ is continuous and continuously differentiable in $[0, \infty)$. Then

$$
\int_{t-h}^{t} y_{0}^{2}(t-s) d s \leq h \sup _{0 \leq \theta \leq 1} y_{0}^{2}(\theta)=C h
$$

and

$$
\left|\Delta_{h} y_{0}(t-s)\right| \leq h\left\{\begin{array}{l}
\sup _{0 \leq \theta \leq 1}\left|y_{0}^{\prime}(t-s+\theta)\right|, \\
t-1 \leq s \leq t ; \\
\sup _{0 \leq \theta \leq 1}\left|y_{0}^{\prime}(t+\theta-s)\right|, \\
s \leq t-1 ;
\end{array} \quad=h\left\{\begin{array}{l}
\sup _{0 \leq u \leq 2}\left|y_{0}^{\prime}(u)\right|, t-1 \leq s \leq t \\
\sup _{0 \leq \theta \leq 1} \mid a y_{0}(t+\theta-s)+ \\
b y_{0}(t+\theta-s-1) \mid, s \leq t-1 .
\end{array}\right.\right.
$$

As follows

$$
\begin{gathered}
A(h) \leq C\left(h+h^{2}\left(\left(\sup _{0 \leq u \leq 2}\left|y_{0}^{\prime}(u)\right|\right)^{2}+\right.\right. \\
\left.\left.+\sup _{0<\theta<1}\left[\int_{-\infty}^{t-1} y_{0}^{2}(t+\theta-s) d s+\int_{-\infty}^{t-1} y_{0}^{2}(t+\theta-s-1) d s\right]\right)\right) \leq \\
\leq C\left(h+h^{2}\left(\left(\sup _{0 \leq u \leq 2}\left|y_{0}^{\prime}(u)\right|\right)^{2}+\int_{0}^{\infty} y_{0}^{2}(s) d s\right)\right) \leq C h .
\end{gathered}
$$

Thus, according to Proposition 2, the assertion of Proposition 3 holds true for the process $\left(Z_{1}(t)\right)$.

The other assertions of Proposition 3

$$
Z_{i}(t)=O\left((\log t)^{\frac{1}{2}}\right), i=\overline{2,4} \text { as } t \rightarrow \infty \quad P_{\vartheta}-\text { a.s. }
$$

follow from the obtained relation and from the definition of the functions $Z_{i}(t), \quad i=$ $\overline{2,4}$.

Hence Proposition 3 is proved.

\section{Acknowledgments}

The authors express their thanks to A. Gushchin for the Proposition 2. 


\section{References}

[1] R. Bellman. Introduction to Matrix Analysis, Mcgraw-Hill Book Company, INC. New York, Toronto, London, 1960.

[2] P. J. Brockwell. Levy-driven CARMA processes. Ann. Inst. Stat. Math., volume 53, pages 113-124, 2001.

[3] L. Galtchouk, and V. Konev. On sequential estimation of parameters in semimartingale regression models with continuous time parameter. The Annals of Statistics, volume 29, 5, pages 1508-1536, 2001.

[4] A. A. Gushchin, and U. Küchler. Asymptotic inference for a linear stochastic differential equation with time delay. Bernoulli, volume5, 6, pages 1059-1098, 1999 .

[5] V. Kolmanovskii, and A. Myshkis. Applied Theory of Functional Differential Equations, Kluwer Acad. Pabl., 1992.

[6] V. V. Konev, and S. M. Pergamenshchikov. Sequential estimation of the parameters of diffusion processes. Problems of Inform. Trans., volume 21, 1, pages 48-62, 1985 (in Russian).

[7] V. V. Konev, and S. M. Pergamenshchikov. Sequential estimation of the parameters of linear unstable stochastic systems with guaranteed accuracy. Problems of Inform. Trans., volume 28, 4, pages 35-48, 1992 (in Russian).

[8] G. Kramer, and M. Leadbetter. Stationary Random Processes. Properties of Sampling Functions and Their Applications. Moscow, 1969.

[9] U. Küchler, and V. Vasiliev. On sequential parameter estimation for some linear stochastic differential equations with time delay. Sequential Analysis, volume 20, 3, pages 117-146, 2001.

[10] U. Küchler, and V. Vasiliev. On sequential identification of a diffusion type process with memory. Proceedings Symp. Int. Fed. Autom. Contr. SYSID-2003, Rotterdam, Holland, 27-29 August, pages 1217-1221, 2003.

[11] U. Küchler, and V. Vasiliev. Sequential identification of linear dynamic systems with memory. Statist. Inference for Stochastic Processes, volume 8, 1, pages 124,2005 .

[12] U. Küchler, and V. Vasiliev. On Sequential Estimators for an Affine Stochastic Delay Differential Equations. Algorithms for Approximation. Proceedengs of the 5th International Conference, Chester, July 2005, Editors: A. Iske, J. Levesley. Springer-Verlag, Berlin, Heidelberg, pages 287-296, 2006.

[13] U. Küchler, and V. Vasiliev. On sequential parameter estimation of a linear regression process. Proceedings of the 17th World Congress The Int. Fed. Autom. Contr., Seoul, Korea, 6-11 July, pages 10230-10235, 2008. 
[14] T. L. Lai, and C. Z. Wei. Least squares estimates in stochastic regression models with applications to identification and control of dynamic systems. Ann. Statist., volume 10, pages 154-166, 1982.

[15] M. A. Lifshits. Gaussian Random Functions. Kluwer Academic Publishers. Dordrecht, Boston, London, 1995.

[16] R. S. Liptzer, and A. N. Shiryaev. Statistics of Random Processes. SpringerVerlag, New York, Heidelberg, 1977.

[17] R. S. Liptzer, and A. N. Shiryaev. Theory of martingales. Kluwer, Dordrecht, 1989.

[18] A. A. Novikov. The sequential parameter estimation in the process of diffusion type. Probab. Theory and its Appl., volume 16, 2, pages 394-396, 1971 (in Russian).

[19] Yu. A. Rozanov. Stationary Gaussian Processes. Holden Day: San Francisco, CA, 1967. Russian original, Moscow, Fizmatgiz, 1963. 